\title{
Bandwidth Oscillations Control and Quality of Service in CDMA-2000 Interconnections
}

\author{
by \\ Tamir Erlichman \\ B.Sc. Electrical and Computer Engineering \\ Ben-Gurion University of the Negev, Israel
}

\begin{abstract}
A thesis submitted to the Faculty of Graduate Studies and Research in partial fulfillment of the requirements for the degree of Master of Applied Science
\end{abstract}

Ottawa-Carleton Institute for Electrical and Computer Engineering Department of Systems and Computer Engineering

Faculty of Engineering

Carleton University

Ottawa, Canada

(C) Tamir Erlichman, 2006 


$\begin{array}{ll}\begin{array}{l}\text { Library and } \\ \text { Archives Canada }\end{array} & \begin{array}{l}\text { Bibliothèque et } \\ \text { Archives Canada }\end{array} \\ \begin{array}{l}\text { Published Heritage } \\ \text { Branch }\end{array} & \begin{array}{l}\text { Direction du } \\ \text { Patrimoine de l'édition }\end{array} \\ \begin{array}{l}\text { 395 Wellington Street } \\ \text { Ottawa ON K1A ON4 }\end{array} & \begin{array}{l}\text { 395, rue Wellington } \\ \text { Ottawa ON K1A ON4 } \\ \text { Canada }\end{array}\end{array}$

Your file Votre référence ISBN: 978-0-494-18313-7 Our file Notre référence ISBN: 978-0-494-18313-7

NOTICE:

The author has granted a nonexclusive license allowing Library and Archives Canada to reproduce, publish, archive, preserve, conserve, communicate to the public by telecommunication or on the Internet, loan, distribute and sell theses worldwide, for commercial or noncommercial purposes, in microform, paper, electronic and/or any other formats.

The author retains copyright ownership and moral rights in this thesis. Neither the thesis nor substantial extracts from it may be printed or otherwise reproduced without the author's permission.
AVIS:

L'auteur a accordé une licence non exclusive permettant à la Bibliothèque et Archives Canada de reproduire, publier, archiver, sauvegarder, conserver, transmettre au public par télécommunication ou par l'Internet, prêter, distribuer et vendre des thèses partout dans le monde, à des fins commerciales ou autres, sur support microforme, papier, électronique et/ou autres formats.

L'auteur conserve la propriété du droit d'auteur et des droits moraux qui protège cette thèse. $\mathrm{Ni}$ la thèse ni des extraits substantiels de celle-ci ne doivent être imprimés ou autrement reproduits sans son autorisation.
In compliance with the Canadian

Privacy Act some supporting forms may have been removed from this thesis.

While these forms may be included in the document page count, their removal does not represent any loss of content from the thesis.
Conformément à la loi canadienne sur la protection de la vie privée, quelques formulaires secondaires ont été enlevés de cette thèse.

Bien que ces formulaires aient inclus dans la pagination, il n'y aura aucun contenu manquant.

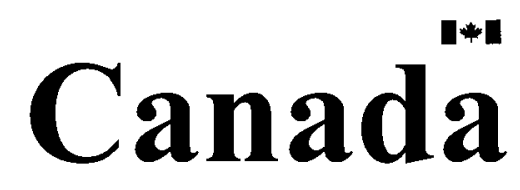




\section{Abstract}

The common 'finite-burst' mode of wireless links scheduling in CDMA-2000 data networks is featured with rate variations causing system bandwidth swings and degraded system performances. The shared nodes at the CDMA-2000 data network are constructed with rate mismatch allowing large traffic variations at the bottleneck node whereas the feeding node is largely underutilized. The CDMA-2000 data network standard offers a single level of service, which cannot differentiate between today's applications' distinct QoS requirements.

We investigate RED performances in the context of CDMA-2000 as a function of its controls' configuration space using both formal mathematics and simulations. RED's transient response is examined using fluid-flow approximation, and a non-linear estimator on the overflow-based drop burst size is derived. We extend previous performance evaluation studies of the bandwidth-swings effects via simulations, allowing various TCP versions as well as unresponsive traffic (UDP) through the system. We identify the system's distinct areas of operation as a function of the bottleneck's RED controls. We perform bottleneck RED queue tune-up via simulation and identify its superior settings.

We evaluate the 3GPP2 proposal of backpressure in CDMA-2000 for protecting the bottleneck queue during congestion scenarios. We propose a feedback flow-control model for tandem nodes at CDMA-2000. We demonstrate the performance benefits and stress the pertaining costs.

We evaluate the DiffServ architecture for CDMA-2000 data-networks. We propose a QoS model that adapts the Diffserv building blocks to the CDMA-2000 distinct architecture and constraints by employing our feedback flow-control mechanism. 


\section{Acknowledgements}

This thesis is the result of two years of work whereby I have been accompanied and supported by various people. In this period I acquired knowledge in computer communication networks and cellular data-networks. I'm greatly thankful for those following people.

The first person I would like to thank is my university direct supervisor, professor Ioannis Lambadaris. I have been under his supervision since September 2004 when I started my Master's of Engineering. I thank him for his patience and encouragement throughout the course of the study. His insights and comments helped to shape my research skills. His invaluable feedbacks contributed greatly to the success of this dissertation.

I want to thank Dr. Parsa Larizani from Nortel Networks, who advised me in various aspects of my research. His in-depth knowledge of CDMA-2000 congestion issues has proven very useful during the thesis. His useful ideas and suggestions have led to the detailed simulation experiments carried out, and assisted in satisfying the thesis topics.

I thank all the students and staffs in the broadband networks laboratory (BNL) in Carleton University, whose presences and study spirits made the otherwise grueling experience tolerable. I was helped with various technical topics, and enjoyed all the vivid discussions we had on various other topics. I enjoyed sharing the convenient and advanced laboratory facilities with them. I give special thanks to Vikas Paliwal for his technical head start at the initial stage of the thesis. I had a great pleasure at being a member of this fantastic group. 
To my family:

Avivit, Nofar, Shir, and Aitan 


\section{Table of Contents}

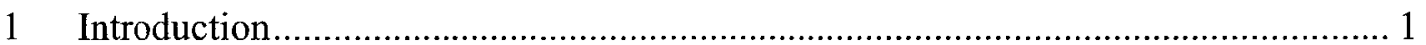

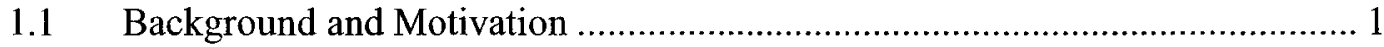

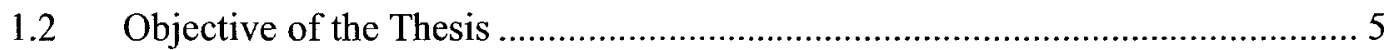

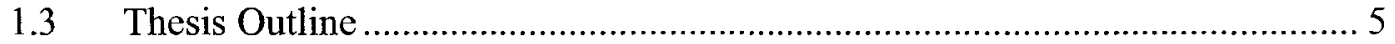

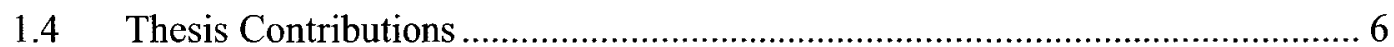

2 Congestion Control in CDMA-2000 Data-Networks ........................................... 9

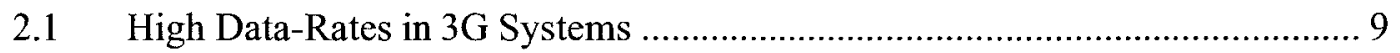

2.2 Feedback Flow-Control in Open-RP Protocol Stack ....................................... 10

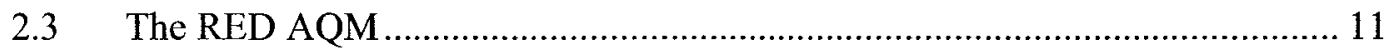

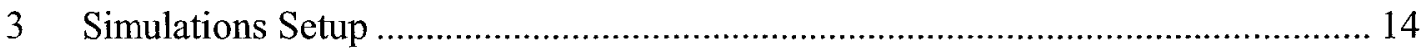

$3.1 \quad$ CDMA-2000 Reference Model................................................................. 14

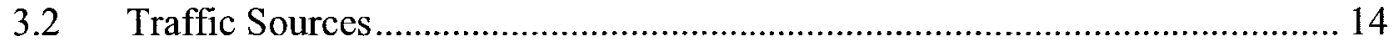

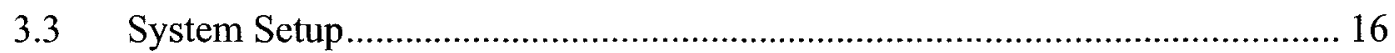

3.4 System and Queuing Performance Metrics of Interest ............................... 17

4 Theoretical Analysis of RED in CDMA-2000 .................................................. 20

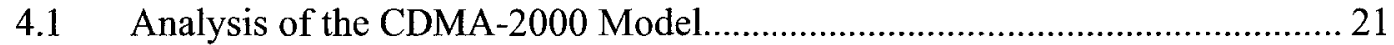

4.1.1 Queuing Model ......................................................................... 21

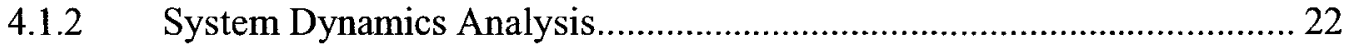

4.1.3 RED Dynamics Analysis at the Context of CDMA-2000 ...................... 25

4.2 Application to CDMA-2000 System Parameters............................................ 33

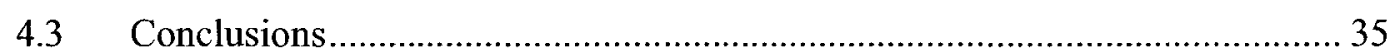

5 Simulation-Based RED Tune-Up for CDMA-2000 …........................................ 36

5.1 Bandwidth Oscillations Extended Evaluation ................................................ 36

5.1.1 Comparative Evaluation of TCP Versions............................................... 36

5.1.2 System Throughput with Reno TCP .................................................... 38

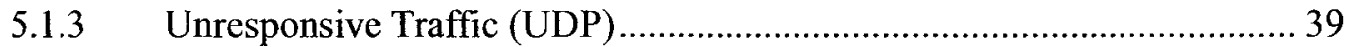




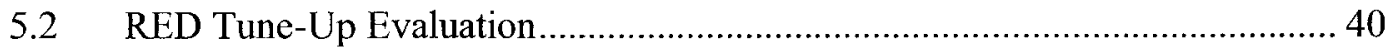

5.2.1 System Boundaries and Areas of Operations......................................... 41

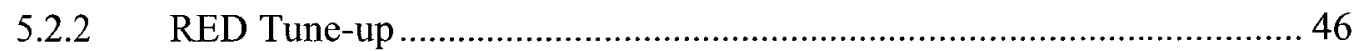

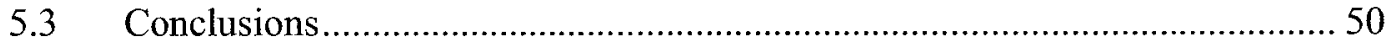

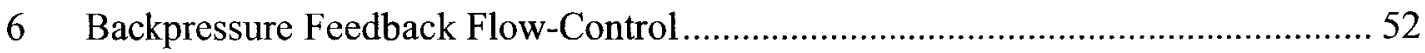

6.1 Description of the Feedback Flow-Control Model ....................................... 53

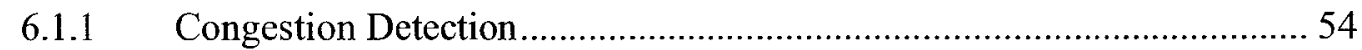

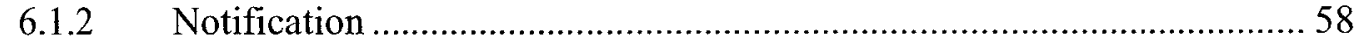

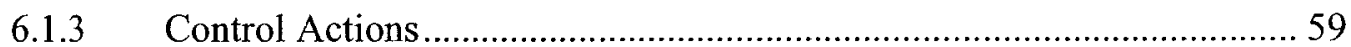

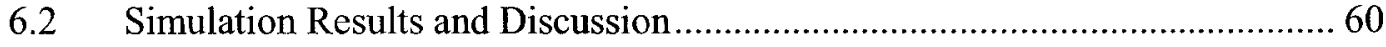

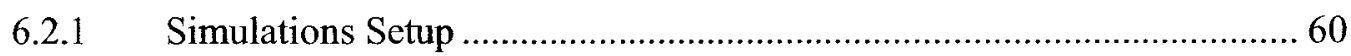

6.2.2 Temporal Queues and System Dynamics .............................................. 62

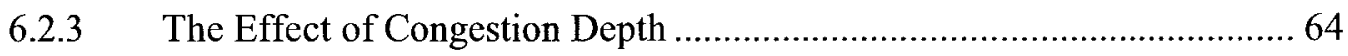

6.2.4 The Effect of Congestion Duration......................................................... 66

6.2.5 Comparison with a Nodal Model of Enlarged Buffer............................. 67

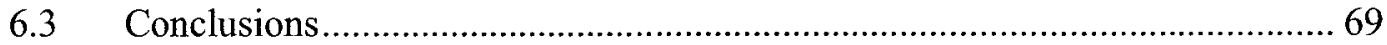

7 QoS Model for Service Differentiation........................................................... 71

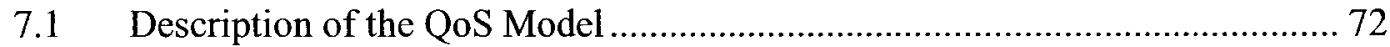

7.1.1 The PCF Node............................................................................. 73

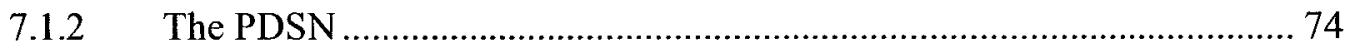

7.1.3 Parameters Setting ................................................................... 75

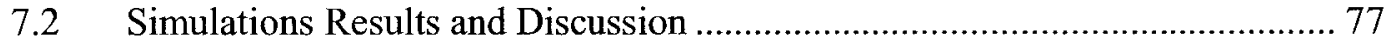

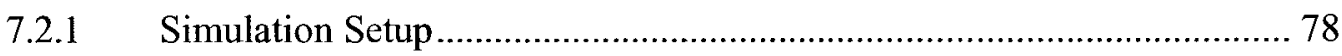

7.2.2 Temporal Queue and System Dynamics............................................. 79

7.2.3 Effect of Congestion Depth .......................................................... 80

7.2.4 Effect of Congestion Duration ........................................................... 82

7.2.5 Effect of Backpressure Close-Loop Delay ...................................... 85

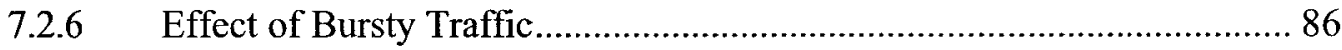

VII 
7.2.7 Effect of Multiple Transport Layers .............................................. 89

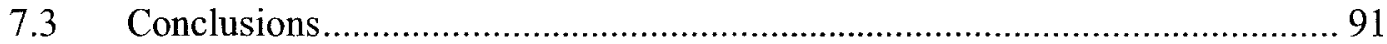

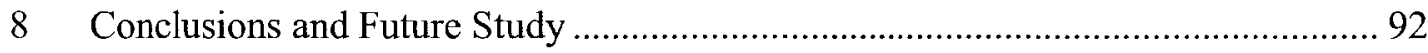

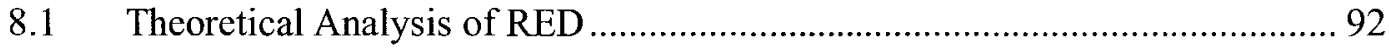

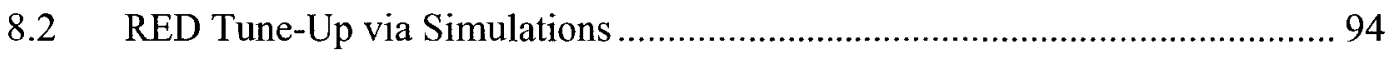

8.3 Backpressure Feedback Flow-Control ...................................................... 95

8.4 Backpressure Enabled QoS Model ....................................................... 97

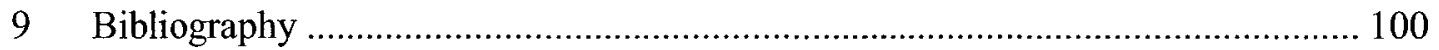




\section{List of Figures}

Fig. 2.1 Simple-IP Open-RP Protocol Stack ..................................................... 10

Fig. 2.2 GRE Header Extension for Feedback Flow-Control ................................. 11

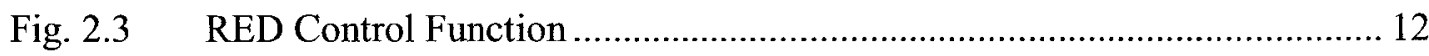

Fig. 3.1 CDMA-2000 Data-System Reference Model ....................................... 14

Fig. 4.1 Queuing Model for Packet-Losses at the Shared-Buffer .......................... 21

Fig. 4.2 Numerical Results for Overflow-based Drops Estimate $L_{V}^{\text {measure }}$ and

Simulations Results vs. Varying: (a) Buffer-Size (b) Thresholds ratio (c) Target-

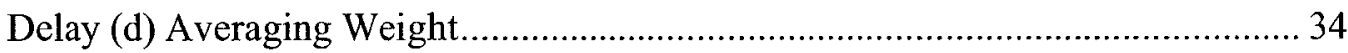

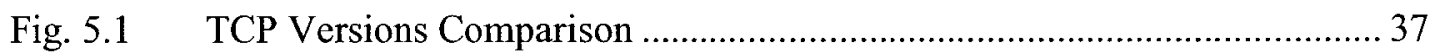

Fig. 5.2 System Throughput with ACK Compression in CDMA-2000 ................... 38

Fig. 5.3 Throughput vs. UDP-to-TCP Connections Ratio ..................................... 40

Fig. 5.4 TCP Performances vs. Bottleneck Queue's Controls (a) Throughput (b)

Delay (c) Jitter (d) Data Packet Drops (e) Power Function .................................... 44

Fig. 5.5 Bias against Bursty TCP Traffic vs. Bottleneck Queue's Controls (a)

Throughput percentage (b) Normalized throughput .......................................... 44

Fig. 5.6 Throughput of TCP and UDP Mix vs. Bottleneck Queue's Controls (a)

Throughput (b) Delay (c) Jitter (d) Data Packet Drops (e) Power Function ............ 46

Fig. 5.7 RED Tune-up Objective Function with TCP (Exploitation Phase) ............ 48

Fig. 5.8 RED Tune-up Objective Function with Traffic Mix of TCP and UDP

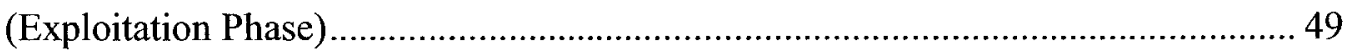

IX 
Fig. 6.1 Architectural View of the Tandem Queues Closed-Loop Feedback Flow-

Control for Single Sided Traffic 53

Fig. 6.2 Difficulty in Tuning Xoff/Xoff Backpressure's Fixed Thresholds.............. 56

Fig. 6.3 Upper and Lower Thresholds Interaction in Adaptive-Xoff/Xon Scheme... 58

Fig. 6.4 Adaptive-Xoff/Xon Control Packet Submission Decision Routine............. 58

Fig. 6.5 Temporal queuing and backpressure dynamics (a) PCF thresholds and

occupancy levels (b) PDSN thresholds and occupancy levels (c) calculated occupancy smoothed rate (d) cumulative data packet drops 63

Fig. 6.6 Temporal queuing delays. 64

Fig. 6.7 Backpressure model's performances benefits as a function of the congestiondepth (a)(b) throughput (c) tandem-queues' data packet drop rate. 65

Fig. 6.8 Backpressure model's performances costs as a function of the congestion depth (a) tandem-queues average-delay during 'burst' intervals (b) overhead of control packets

Fig. 6.9 Backpressure model's performances benefits as a function of the congestionduration (a)(b) throughput (c) tandem-queues' data packet drop rate 66

Fig. 6.10 Backpressure model's performances costs as a function of the congestion duration (a) tandem-queues average-delay during 'burst' intervals (b) overhead of control packets

Fig. 6.11 Comparison of backpressure model with nodal model of enlarged buffer size, function of congestion depth: (a) throughput (b) tandem-queues average-delay during 'burst' intervals.

Fig. 6.12 Comparison of backpressure model with nodal model of increased buffer size function of congestion duration: (a) throughput (b) tandem-queues averagedelay during 'burst' intervals (c) Tandem power $(=(a) /(b))$

Fig. 7.1 CDMA-2000 DiffServ Architecture with Feedback Flow-Control 73 
Fig. 7.2 Temporal PCF and PDSN queuing and backpressure dynamics (a) wireless link bandwidth and throughput (b) PCF and PDSN RED queues commutative drops (c) PCF RED queues occupancy dynamics

Fig. 7.3 QoS model performance as a function of the congestion depth (a)(b) system and class-based throughputs (c) average delay during congestion (d) percentage of data packet loss volume at the PDSN per traffic class (e) Data packet loss volume distribution between the PCF and the PDSN (f) backpressure control packet overhead

Fig. 7.4 QoS model performance as a function of the congestion duration (a)(b) system and class-based throughputs (c) average delay during congestion (d) percentage of data packet loss volume at the PDSN per traffic class (e) Data packet loss volume distribution between the PCF and the PDSN (f) backpressure control packet overhead

Fig. 7.5 QoS model performance as a function of the backpressure close-loop delay (a) system and class-based throughputs (b) average delay during congestion (c) percentage of data packet loss volume at the PDSN per traffic class (d) Data packet loss volume distribution between the PCF and the PDSN (e) backpressure control packet overhead

Fig. 7.6 QoS model performance as a function of the backpressure close-loop delay (a) system and class-based throughputs (b) average delay during congestion (c) percentage of data packet loss volume at the PDSN per traffic class (d) Data packet loss volume distribution between the PCF and the PDSN (e) backpressure control packet overhead 88

Fig. 7.7 QoS model's throughput as a function of the UDP application rate (a) PDSN with 2 distinct queues (b) nodal model of a single service level .89

Fig. 7.8 QoS model performance as a function of the UDP application rate (a) average delay during congestion (b) percentage of data packet loss volume at the 
PDSN per traffic class (c) Data packet loss volume distribution between the PCF and the PDSN (d) backpresure control packet overhead 90 


\section{List of Tables}

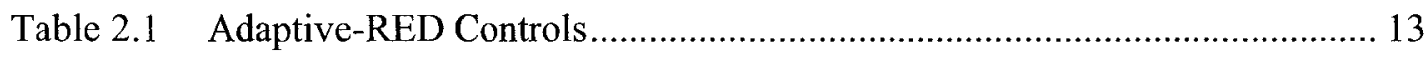

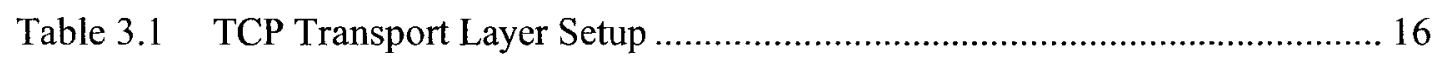

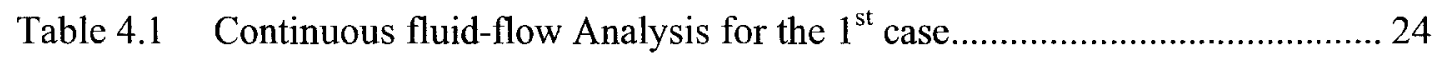

Table 5.1 RED Tune-Up Highest Results with TCP ........................................... 49

Table 5.2 RED Tune-Up Highest Results with Traffic Mix of TCP and UDP.............50 


\section{List of Abbreviations}

\begin{tabular}{ll} 
3G & Third Generation \\
3GPP2 & Third Generation Partnership Program 2 \\
ACK & Acknowledgement Packet \\
AQM & Active Queue Management \\
ARQ & Automatic Repeat Request \\
BA & Behavioral Aggregate \\
BHL & Back Haul Link \\
BP & Backpressure \\
BSC & Base Station Controller \\
BTS & Base Transceiver Subsystem \\
CBR & Constant Bit Rate \\
CDMA & Code Division Multiple Access \\
DBP & Delay Bandwidth Product \\
DiffServ & Differentiated Services \\
DS & DiffServ \\
DSCP & DiffServ Code Point \\
CWND & Congestion Window \\
EXP & Exponential \\
FCH & Fundamental Channel \\
FTP & File Transfer Protocol \\
GRE & Generic Routing Protocol \\
IETF & Internet Engineering Task Force \\
IP & Layer 1 \\
\hline L & Arotocol \\
\hline
\end{tabular}




\begin{tabular}{ll} 
Mbps & Mega Bit Per Second \\
MS & Mobile Station \\
MSS & Maximum Segment Size \\
NS2 & Network Simulator version 2 \\
Open-RP & Open RAN to PDSN protocol stack \\
PCF & Packet Control Function \\
PDSN & Packet Data Service Node \\
PHB & Per-Hop Behavior \\
PPP & Point to Point Protocol \\
QoS & Quality of Service \\
RAN & Radio Access Network \\
RED & Random Early Detection \\
RF & Radio Frequency \\
RLP & Radio Link Protocol \\
RS & Rate-Set \\
RTO & Retransmission Timeout \\
RTT & Round Trip Time \\
SACK & Selective ACK \\
SCH & Supplemental Channel \\
SIG & Signaling \\
SLA & Services Level Agreements \\
TCP & TCP Transmission Control Protocol \\
UDP & Uatagram Protocol \\
WR & Teund Robin \\
\hline
\end{tabular}




\section{List of Notations}

\section{Theoretical analysis of RED in CDMA-2000:}

$\begin{array}{lll}q_{\text {ave }} & \text { RED queue average size } & 11\end{array}$

$\begin{array}{lll}w_{q} & \text { RED queue averaging weight } & 11\end{array}$

$q_{\text {inst }} \quad$ Queue instantaneous size $\quad 11$

$\begin{array}{lll}n & \text { The number of packet arrival } & 11\end{array}$

$\begin{array}{ll}t h_{\min } & \text { RED lower threshold } \\ & 12\end{array}$

$t h_{\max } \quad$ RED upper threshold 12

$\begin{array}{lll}p_{b} & \text { RED initial probability of packet dropping } & 12\end{array}$

$\begin{array}{lll}p_{\max } & \text { RED maximum packet drop probability } & 12\end{array}$

$\begin{array}{lll}\text { count } & \text { Number of packets since the last drop }\end{array}$

$\begin{array}{lll}p_{a} & \text { RED final dropping probability } & 12\end{array}$

$q_{\text {trg }} \quad$ RED target queue size during congestion $\quad 13$

$\begin{array}{lll}C & \text { Link capacity } & 13\end{array}$

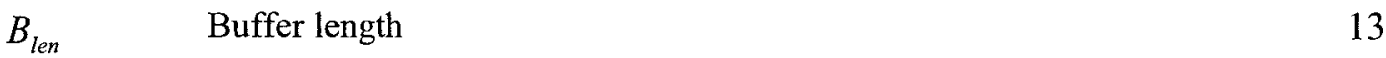

ths $s_{\text {ratio }} \quad$ The ratio between the upper and lower thresholds of RED 13

$\begin{array}{lll}r_{P C F} & \text { The PCF service rate } & 16\end{array}$

$\begin{array}{lll}\eta_{l l} & \mathrm{FCH} \text { rate } & 21\end{array}$

$\begin{array}{lll}\eta_{l h} & \mathrm{FCH} \text { and } \mathrm{SCH} \text { aggregated rate } & 21\end{array}$

$\begin{array}{lll}B_{s} & \text { The shared-queue buffer size } & 21\end{array}$

$\begin{array}{lll}\eta_{S} & \text { The shared-queue service-rate } & 21\end{array}$

$q_{i}^{L}(t) \quad$ The link-buffers' aggregated occupancy at time instance $i$

$q_{i}^{S}(t) \quad$ The PCF shared-buffer occupancy at time instance $i$ 
$\begin{array}{lll}B_{L} & \text { The size of each link queue's buffer } & 21\end{array}$

$\begin{array}{ll}t_{i} \quad \text { Time instance } i & 22\end{array}$

$\begin{array}{lll}W & \text { TCP congestion window size } & 22\end{array}$

$\begin{array}{lll}T & \text { Round trip time (excluding queuing delays) } & 22\end{array}$

$t_{\text {start }} \quad$ The time instance in which the leading edge of packets at higher rate reaches the shared buffer $\quad 22$

$t_{\text {end }} \quad$ The time instance in which the leading edge of packets at higher rate ends at the shared buffer $\quad 22$

$t_{\text {end }}^{I, I I I I} \quad$ The time instance in which the leading edge of packets at higher rate ends at the shared buffer for either case I, II, or III 22

$\mathrm{q}_{\mathrm{EDI}}^{\mathrm{s}} \quad$ The shared-queue occupancy at the time-instance in which the first probabilistic early-drop may occurs $\quad 22$

$\begin{array}{lll}E D & \text { The volume of early drops before the queue overflows } & 23\end{array}$

$H_{\text {ave/inst }}$ The transfer function of the RED queue averaging low-pass filter 26

$h_{\text {inst } / \text { in }} \quad$ The relationship between the shared-queue's inflow rate and its instantaneous size growth-rate 26

$\begin{array}{lll}U[n] & \text { Unit step function } & 26\end{array}$

$\Phi_{\text {trg }} \quad$ The RED queue's target occupancy during congestion, presented as a fraction of the RTT (i.e., $q_{t r g}=\Phi_{t r g} \cdot R T T$ ) 27

$p_{C F} \quad$ Continuous flow approximation to RED's packet drop probability 28

$n_{\text {end }} \quad$ The smallest number of packets in which the shared-queue overflows or the high-fluid inflow ceases $\quad 30$

$n_{\text {start }} \quad$ The largest number of packet arrivals at the shared-queue before the first possible packet drop $\quad 29$ The largest number of packet arrivals at the shared-queue before the average queue size crosses the lower threshold 
$n_{O F} \quad$ The number of packet arrivals at the shared-queue at the start of the overflow-based packet drops $\quad 30$

$R_{\text {OFdrop }}^{\text {measure }} \quad$ The estimate on the measured volume of the overflow-based packet drops during buffer overflow interval 31

$R_{\text {edrop }}^{\text {mease }} \quad$ The rate of early drops during overflow period at the shared-queue

$L_{V}^{E D @ O F} \quad$ The volume of early drops throughout the overflow period at the shared-queue

$n_{\text {end }}^{E D @ O F} \quad$ The number of packet arrivals at the shared-queue in which the fluid of packets at higher rate ceases $\quad 32$

$L_{V}^{\text {measure }} \quad$ An estimate on the volume of overflow-based packet losses at a single bandwidth-swing related burst of traffic in RED implementation at NS2

\section{RED tuning using simulations:}

Obj The objective function for RED tuning via simulations

\section{Backpressure feedback flow-control:}

$D_{\text {loop }} \quad$ The backpressure's feedback loop delay 54

$D_{\text {prop }} \quad$ The PCF to (and from) PDSN internodes propagation delay 54

$L_{c n t r} \quad$ The size of a backpressure's control packet 54

$\triangle Q_{P C F}^{X o f f} \quad$ The potential PCF queue occupancy increase after the backpressure's upper threshold was exceeded and an Xoff feedback indication was sent

$R_{\text {mismatch }}^{\text {Xon }} \quad$ The rate mismatch between the PCF's inflow and outflow during Xon interval at the PDSN

$\Delta Q_{P C F}^{X o n} \quad$ The potential PCF queue occupancy decrease after the

\section{XVIII}


backpressure's lower threshold was crossed down and an Xon

feedback indication was sent

$R_{\text {mismach }}^{\text {Xoff }} \quad$ The rate mismatch between the PCF's outflow and inflow during Xoff interval at the PDSN 55

$B_{P C F} \quad$ PCF's buffer size $\quad 55$

$r_{Q} \quad$ The PCF's occupancy smoothed growth-rate 56

$r \quad$ The PCF's occupancy instantaneous growth-rate 56

$q_{S}^{\text {inst }} \quad$ The PCF's buffer instantaneous occupancy 57

$t h_{\text {target }}^{\text {Xoff }} \quad$ The target maximum buffer fill at the PCF, enforced by the

$\begin{array}{ll}\text { backpressure } & 57\end{array}$

$t h_{\text {gen }}^{\text {Xof }}[i] \quad$ The backpressure's discrete time adaptive upper threshold (Xoff feedback indication generation) $\quad 57$

$t h_{t \text { arget }}^{\text {Xon }} \quad$ The target minimum buffer fill at the PCF, enforced by the $\begin{array}{ll}\text { backpressure } & 57\end{array}$

$t h_{\text {gen }}^{X o n} \quad$ The backpressure's lower threshold (Xon feedback indication generation) $\quad 57$

C The center of the backpressure-based queue occupancy variations $\quad 57$

$H \quad$ The size of the backpressure-based queue occupancy variations $\quad 57$

$U \quad$ The backpressure's control role at the feeding node (PDSN) 59

$\Delta t_{\text {PDSN }}^{\text {elapse }} \quad$ The Xoff elapse time at the feeding node (PDSN) 59

QoS model for service differentiations:

$B W_{e f f}^{Q}{ }^{Q} i \quad$ The effective rate of a particular class queue at a multi-queues DiffServ architecture with bandwidth scheduling at the PDSN $\quad 76$

$\begin{array}{ll}B W_{P C F} & \text { The PCF's out-link bandwidth }\end{array}$ 
$W R R_{w e i g h t}^{Q}{ }^{i} \quad$ The WRR weight associated with physical queue $i$ (at the PDSN)

$q_{t a r g e t}^{Q_{i} i} \quad$ RED's target queue occupancy during congestion for physical queue $i$ in multi-queues DiffServ architecture

$D_{t \text { arget }}^{Q_{i} i} \quad$ RED's target average delay during congestion for physical queue $i$ in multi-queues DiffServ architecture

$t h_{\min }^{Q_{-i}}$ RED's lower threshold for physical queue $i$ in multi-queues DiffServ architecture

$t h_{\max }^{Q_{-} i}$ RED's upper threshold for physical queue $i$ in multi-queues DiffServ architecture

ths $s_{\text {ratio }}^{Q_{i} i} \quad$ RED's thresholds ratio for physical queue $i$ in multi-queues DiffServ architecture

size $_{\text {buffer }}^{Q-i} \quad$ The buffer size of physical queue $i$ in multi-queues DiffServ architecture

$w_{q}^{Q_{-} i} \quad$ RED's queue averaging weight for physical queue $i$ in multiqueues DiffServ architecture 


\section{Introduction}

\subsection{Background and Motivation}

The CDMA-2000 cellular data-networks are featured with large delay and bandwidth variations over the wireless links. In the finite-burst mode wireless channel scheduling supplemental channel $(\mathrm{SCH})$ is periodically assigned for a user in addition to an already allocated fundamental channel (FCH) [2][3]. The vastly used transport-protocol in these data-networks, the TCP protocol, was designed for reliable data transmission across wired networks with rather fixed topology. This combination results in performance degrading effects at the wireless data-networks, which were identified to be an important factor in reducing the system performance.

Analytical studies of CDMA-2000 cellular rate increase with SCHs allocations were performed at [4] and [5] for the case of the simple Tail-Drop queue management discipline at the shared-queue. These studies showed ACK-compression effect at the linkbuffers and subsequent large burst of data-packets drops at the shared queue once SCHs are allocated closely in time. The extensive losses volume follows by system global synchronization where multiple TCP senders concurrently decrease their rate, and subsequent shared-queue occupancy instability and system underutilization periods. The popular Reno TCP version in particular was identified at [1] with an extremely low probability to recover by fast re-transmit after three subsequent packet losses, whereas 4 packet losses or more requires the costly RTO-based recovery with probability one. A network-based solution of ACK Regulator was suggested at [5] to mitigate the effect of rate variability. This solution showed an improvement in TCP throughput, however with the costs of increased round trip time and increased complexity.

The linear analysis for a Tail-Drop disciplined shared queue at CDMA-2000 bottleneck performed at [4] did not address the more commonly used and practical queue disciplines. On the other hand, analytical models proposed for RED [14][13] addressed a 
steady topology of the wire-Internet with a fixed and single bottleneck, where steady state analysis was largely analyzed. However, the excessive traffic bursts due to the ACKcompression phenomena and the short the SCH burst durations call for transient response analysis. In this study we provide a formal mean to theoretically evaluate the transientresponse of today's de-facto queue management, the RED AQM, as a function of its configuration space, at the context of the CDMA-2000 data-networks.

Today de-facto RED [6] active queue management mechanism for data-networks' gateways was designed to provide superior performance over the traditional Tail-Drop discipline. RED detects the inception of congestion and notifies it to the traffic sources to avoid serious congestion. However, its parameter setting has proved to largely affect it performance [11], specifically under the scenario of variable traffic loads. Analytical models of RED in the presence of TCP traffic for the wire-Internet with steady topology, fixed service-rate queues, and fixed system bottleneck, were introduced to provide some guidelines for setting RED parameters [14][13]. Adaptive-RED [10] was further proposed to allow an a-prior average delay at the queue with slow variability of traffic load. Tuning of RED discipline for the wired-Internet topology using simulation was performed at [11][15] [12].

At [4] simulation-based performance evaluation was done for a shared-node with RED queue to show the bandwidth-swings effects. However, the investigation there concluded the system behavior using a RED mechanism carrying a single set of configuration for all the simulations. This configuration followed the recommendations for the wired-Internet at the literature [6][10]. The literature setting guidelines assume a rather fixed networks topology resulting with a fixed bottleneck queue. Conversely, in the cellular domain the wireless links' frequent rate changes result with bottleneck shift. In this study we provide an evaluation via simulations of the RED mechanism effectiveness in the context of the CDMA-2000 data-networks as a function of its configuration space (i.e., RED controls).

The nodes with shared-queues at the CDMA-2000 data network are constructed with rate mismatch. The core node (the PCF) is processing power limited where additional 
processing load at the PCF is undesirable. The feeding node (the PDSN) has superior processing and buffering capabilities. Consecutively, a closed-loop inter-nodes feedback flow-control can provides a complement solution to the above AQM flow-control. In this approach a congested node informs its feeding node of the unavailability of buffering capacity and in effect stops it from forwarding more packets until enough buffer become available. For instance, a congested node can insist that no packets are dropped due to buffer overflow events.

Considering the rate mismatch and buffer size differences at the CDMA-2000 nodes, the use of an Xoff/Xon feedback flow control was proposed at the 3GPP2 [17][18] to protect the lower rate core node during wireless links events and traffic sources' load variability. The Xoff/Xon binary feedback flow-control was shown to act as a simple and efficient hop-by-hop backpressure [21][22] [26]. At the Xoff/Xon method, the congested node sends an 'off' signal to feeding nodes when the buffer reaches a high-watermark and sends an "on" signal subsequently when the buffer content has dropped below the low-water mark due to departing packets. When a source receives an "off" signal from the node, it stops transmitting jobs and later resumes transmission upon receiving an "on" signal.

Performance evaluation of tandem nodes backpressure in the context of CDMA-2000 that follows the $3 \mathrm{GPP} 2$ forum recommendations has yet to be performed to our knowledge. The above studies on feedback flow-control, as well as other papers (e.g., [19] [20] [23][24] [25]), introduced limited information, which constrain their applicability to the case of CDMA-2000. They considered either only one or two of today's common combination of flow-controls; namely, the end-to-end flow-control, the inter-nodes flow control, and a nodal active queue management (AQM). They mainly focus on the steady-state behavior of the network. Furthermore, they consider a fully hopby-hop feedback flow-control enabled network; hence introduce over-scaled solutions. In this study we propose and evaluate via simulations the benefits and costs of a tandem nodes backpressure for CDMA-2000 data-networks. 
New applications often require "better" service than single level of service provided by the current IP and wireless networks. This new requirement called for an architecture that can support multiple levels of service while preserving scalability and simplicity. The Differentiated-Services (DiffServ) architecture [29] has been proposed as a scalable solution. DiffServ provides different level of network services by employing a set of well-defined building blocks [30]. The mechanism uses a small label (the DSCP) in the IP layer to determine that a packet is to receive a particular PHB (Per-Hop Behavior) forwarding treatment at each network node. Routers at the domain boundaries enforce the services level agreements (SLAs) by including functionality such as traffic conditioning, monitoring and packet classification, in addition to providing the PHB requirements. Within the core of the network, packets are forwarded according to the PHB associated with the DSCP assigned by the edge router.

DiffServ architecture effectiveness and scalability properties were shown in the literature for the wired-Internet [31][32]. However, the CDMA-2000 cellular datanetwork presents a specific architecture and constraints. In particular, the shared queue at the PCF core node of the CDMA-2000 represents the bottleneck of the system during overload scenarios. Therefore, it is of interest for deploying Diffserv PHB building blocks. This core node is processing limited and its level of complexity is desired to maintain. In this study we propose and evaluate the effectiveness of applying a QoS model, and in particular the DiffServ architecture using backpressure, for CDMA-2000 data-networks under the system constraints.

Today's CDMA-2000 interconnections carry non-congestion-controlled data traffic (e.g., UDP) for a range of applications. The presence of the non-adaptive traffic together with adaptive traffic (e.g., TCP), and specifically with the current single level of handling different traffic types, the system relies on only the transport protocol to react. This scenario presents various negative impacts ranging from extreme unfairness against competing TCP traffic to the potential congestion collapse in the data system [9]. This is because TCP back off when their packets are dropped, whereas the UDP do not react to 
dropping of packets. In this study we evaluate the cross-effect between the TCP and UDP in CDMA-2000 with the various incorporated and proposed flow-controls.

\subsection{Objective of the Thesis}

This thesis provides the devising and performance evaluation of various flow-control solutions in CDMA-2000 data-networks for enhancing performances and providing QoS in the face of the bandwidth swings effects. The specific objectives of the thesis are:

- Tune-up and evaluating the RED performances with CDMA-2000 wireless links scheduling effects as a function of its controls' configuration space:

- By a mean of formal mathematical characterization

- By means of system simulations

- Evaluating the 3GPP2 proposal of backpressure in CDMA-2000 for single class of traffic

- Devise and propose a dedicated tandem nodes backpressure mechanism

- Model the backpressure mechanism in $\mathrm{C}++$ and $\mathrm{Tcl}$ using the NS-2 simulator

- Evaluate the backpressure mechanism's cost effectiveness and performances for protecting the bottleneck node during excessive congestion scenarios

- Evaluate the enhancement of CDMA-2000 data-networks with QoS, and in particular with the DiffServ architecture and backpressure

- Devise and propose a QoS mechanism with backpressure, which addresses the CDMA-2000 specific system architecture and constraints

- Model the QoS mechanism in $\mathrm{C}++$ and Tcl using the NS-2 simulator

- Evaluate the model's cost effectiveness and performances in providing service differentiation for multiple classes of traffic

\subsection{Thesis Outline}

The rest of the thesis document is organized as follows. 
Chapter 2 introduces the structure of the CDMA-2000 networks of high data-rates. It briefs the CDMA-2000 congestion and flow control elements of interest in the thesis.

Chapter 3 describes the simulation setups that are common for the different chapters in this thesis. It depicts the CDMA-2000 reference model, provides the used traffic sources and system setup, and details the performance metrics of interest.

Chapter 4 formally analyzes RED tune-up for mitigating the CDMA-2000 wireless links' rate variations impact. It evaluates the system-system dynamics at the face of the ACK-compression, and derives RED equations specific for our model. It presents a nonlinear expression for the losses-volume estimate, and demonstrates the applicability of the theoretical model with numerical solutions.

Chapter 5 presents performance evaluation of the CDMA-2000 as a function of the RED queue controls. It first evaluates the various TCP versions, as well as different transport layers, in the context of CDMA-2000 model. It then evaluates the CDMA-2000 RED shared-queue area of operation, and tune RED controls for high performance.

Chapter 6 evaluates the 3GPP2 proposal for feedback flow-control in CDMA-2000 data-networks. It first devises a dedicated backpressure mechanism, the AdaptiveXoff/Xon, describing its algorithms and setting criteria. It then presents experimental results of the backpressure in a CDMA-2000 reference model.

Chapter 7 proposes and evaluates a QoS model for service differentiation in CDMA2000. It first depicts the QoS models and its main components: the Adaptive-Xoff/Xon backpressure and the DiffServ architecture. It describes their interoperation and required settings. It then presents performance evaluation of the model using simulations.

Lastly, in section 8 we finalize with the study summary, discussion of the results, conclusions, and suggested directions for future study.

\subsection{Thesis Contributions}

The main contributions of the thesis are as follows: 
- An analytical model of the RED AQM in CDMA-2000 data-networks with finiteburst mode of wireless links scheduling is developed. The model quantifies the effect of the RED controls tuning for any given set of CDMA-2000 system parameters and traffic parameters. A set of non-linear equations is derived using continuous fluidflow approximation. An estimate on the volume of overflow-based burst of drops is presented.

- Numerical results for the estimated volume of overflow-based drop as a function of the RED controls are produced for a given set of system and traffic parameters. The results show the setting ranges for the RED controls that are not recommended, which are characterized with excessive packet loss volume. The results distinguish those from setting ranges for the RED controls that incur low (or non) packet losses. The numerical results are validated with simulations of an equivalent system.

- Analysis via simulations of the RED performances in CDMA-2000 data-networks is presented. In particular, the analysis compares the performance of the most common flavors of the TCP, and examines the cross effect between TCP and UDP traffic under the CDMA-2000 bandwidth swings.

- RED is examined via simulations as a function of controls tuning. The effect of its controls tuning is demonstrated with a range of system and queuing performances. The system performances boundaries and areas of operations for RED are identified. The superior tuning area of RED controls is deduced.

- An evaluation of the 3GPP2 proposal for Xon/Xoff backpressure in CDMA-2000 data-networks is presented. We propose an Adaptive-Xoff/Xon feedback flow-control model, which is based on the Xoff/Xon mechanism. The model aims to complement and assist the RED mechanism at the bottleneck queue during scenarios of high variability of the traversing traffic, such as during bandwidth-swings. A simulation model of the Adaptive-Xoff/Xon is implemented in C++ under the NS-2 simulator. 
- The simulation results of the Adaptive-Xoff/Xon backpressure model present the tradeoff between its benefits and costs. The model eliminates the occurrences overflow-based packet drops during the bandwidth swings, reduces the data packets drop rate, and improves the system throughput. Increased average delay is observed due to the use of free buffer space at the feeding node. The average delay increase is percentage-wise larger than the throughput improvement, which suggests that the 3GPP2 proposal degrades the combined set of delivered performances.

- We propose a QoS model for service differentiation in CDMA-2000 data-systems. The model adapts the DiffServ architecture to the CDMA-2000 architecture and constraints. It uses the Adaptive-Xoff/Xon backpressure to push congestion from the processing limited core node towards the edge node, where differentiated services algorithms can be carried out. The model provides service differentiation in term of the relative portion of access to the shared out-link, and the distinct handling of each traffic class at a dedicated physical RED queue. A simulation model of the QoS model is implemented in $\mathrm{C}+\mathrm{H}$, and incorporated in the NS-2 simulator.

- An evaluation of the QoS model is presented. The effectiveness of the QoS model is demonstrated with various overload levels, congestion depths and durations, traffic sources types, as well as topologies. The model shows to provide throughput and drop rate differentiation between multiple classes of traffic. Low volume of control packet is required to stabilize the core node. We further present an evaluation of the effect of concurrent TCP and UDP transport layers flowing through the system. The results show that the model mitigates the adverse cross effect between TCP and UDP traffic, allowing for a more fair bandwidth share for the TCP traffic. 


\section{Congestion Control in CDMA-2000 Data-Networks}

This chapter describes the high data rates CDMA-2000 network and its congestion and flow control essentials, which are of interest in the thesis.

\subsection{High Data-Rates in 3G Systems}

The higher data rates provided by $2.5 / 3 \mathrm{G}$ wireless systems are made possible by the provision of a new supplemental channel $(\mathrm{SCH})$ to each user in addition to the fundamental channel $(\mathrm{FCH})$. The SCH can provide a peak data rate of $153.6 \mathrm{kbps}$ (in CDMA-2000 Release 0) and 307.2 Kbps (in release A). The FCH typically carries control and signaling information, and can also carry the user data. The SCH, on the other hand, exclusively carries user data. The rate of the allocated SCH is presented as a multiplier of the $\mathrm{FCH}$ with discrete values of power of 2 ranging from $1 \times$ to $32 \times$, where the rate of base FCH is 9.6Kbps for RS (Rate-Set) 1, and 14.4Kbps for RS-2.

There are two types of SCH assignments: 'finite' and 'infinite' [3], which are to be referred as 'finite burst' and 'infinite burst', respectively. Infinite burst means that SCH can be used for transmitting data until a release command is issued. The time interval in which the SCH is allocated is referred to as burst-time, while the inter-bursts time interval between consecutive SCH allocations is referred to as delay-time. Finite burst mode of operation limits the $\mathrm{SCH}$ usage to one of fourteen finite time intervals between $20 \mathrm{msec}$ and 5.12 seconds before it must be released [3].

The CDMA-2000 data-networks structure in interest consists of the following set of nodes. Mobile-stations (MS's) are connected to a base transmitter station (BTS) through wireless links. The BTS provides the radio resources and maintain radio links to mobile station. Multiple BTSs are then communicating with a single base station controller (BSC) through dedicated RF links. The BSC, which provides control and management for one or more BTSs, is connected to a single packet control function (PCF). The PCF, 
contain the shared buffers. The PCFs is then communicating with a PDSN. The PDSN is the gateway between the cellular wireless system and the wired Internet network.

\subsection{Feedback Flow-Control in Open-RP Protocol Stack}

The CDMA-2000 data-network's protocol stack is the simple-IP Open-RP (RAN to PDSN) model. The application-layer and the transport-layer are terminated at the MS and at the Internet hosts. The IP layer is terminated at the MS, at the PDSN for routing, and at the hosts. The PPP, which is established at the initial stage of the connection set-up, provides mechanisms such as: header compression, payload compression and encryption. The RLP protocol comes to mitigate errors on the wireless medium by using fragmentation and reassembly of packets and automatic repeat request (ARQ) schemes. The R-P protocol layer is encapsulated using a GRE header [16], which constitutes an A10/A11 virtual connection per connection. The A10 carries the data (i.e., bearer), whereas the A11 carries the virtual-connections' signaling. Link and network layer frames are carried between the PCF and the PDSN in both directions encapsulated in GRE packets using the A10 connection.

\begin{tabular}{|c|}
\hline $\begin{array}{c}\text { FTP/Telnet/ } \\
\text { VBR }\end{array}$ \\
\hline TCP/UDP \\
\hline IP \\
\hline PPP \\
\hline RLP \\
\hline MAC \\
\hline CHAN \\
\hline
\end{tabular}

Mobile

\begin{tabular}{|c|c|}
\hline MAC & BHL \\
\hline CHAN & TI \\
\hline
\end{tabular}
BTS

\begin{tabular}{|c|c|}
\hline \multirow{2}{*}{$\mathrm{RLP}$} & $\mathrm{RP}$ \\
\cline { 2 - 2 } & $\mathrm{IP}$ \\
\hline $\mathrm{BHL}$ & Ethemet \\
\hline $\mathrm{Tl}$ & $\mathrm{Ll}$ \\
\hline
\end{tabular}

BSCPCF

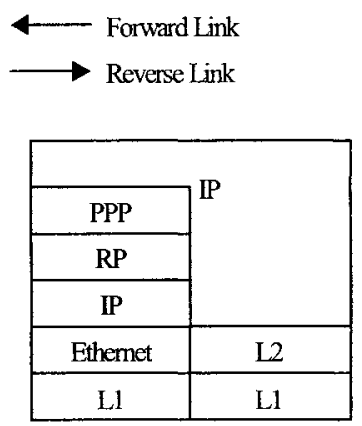

PDSN

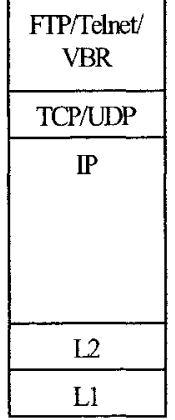

Host

Fig. 2.1 Simple-IP Open-RP Protocol Stack

The use of the GRE and RP protocol allows flow-control indications (e.g., backpressure) to be carried. It uses small number of additional fields incorporated at the GRE header shown at Fig. 2.2. A new GRE Protocol Type, denoted as '3GPP2', is identified for this purpose. The GRE packets' source and destination fields carry the PCF 
and PDSN addresses, and the key field carries the session identifier that indicates which bearer's connection a particular packet is belong to. The attribute fields is included if the protocol-type is set to $3 \mathrm{GPP} 2$ packet, with a length of zero or more octets, and contains feedback flow-control information specific to the attribute. The attribute's Type and Length fields determine the format and length of the Value field. Multiple attributes can be incorporated in a GRE packet, where the $E$ bit fields is set to one for the last attribute [17].

\begin{tabular}{|c|c|c|c|c|c|}
\hline$|\mathrm{C}||\mathrm{K}| \mathrm{S} \mid$ & Reserved & | Ver | & Protocol Type $=3 \mathrm{GPP} 2$ & 1 & \multirow{3}{*}{$3 G P P 2$ GRE R-P packet } \\
\hline \multicolumn{5}{|c|}{ Key } & \\
\hline \multicolumn{5}{|c|}{ Sequence Number (Optional) } & \\
\hline 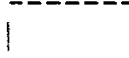 & & Attributes & & & \\
\hline
\end{tabular}

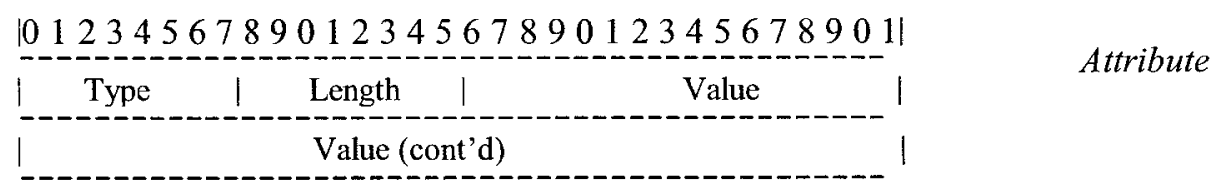

Fig. 2.2 GRE Header Extension for Feedback Flow-Control

\subsection{The RED AQM}

The RED algorithm presents an active queue management (AQM) for packet-switched networks [6]. It is commonly deployed in CDMA-2000 nodes with shared-queues for congestion control. Incipient congestion is detected using monitoring and computing of the queue's average occupancy. Upon congestion, the mechanism notifies connections of that condition by either dropping or marking (setting a bit at the packet's header) packets.

RED employs a low-pass filter to estimate the queue average-size. The calculation of the average queue size (denoted as $q_{\text {ave }}$ ) follows [6]:

$$
q_{\text {ave }}[n+1]=\left(1-w_{q}\right) \cdot q_{\text {ave }}[n]+w_{q} \cdot q_{\text {inst }}[n+1]
$$

where $q_{\text {inst }}$ is the instantaneous queue-size, and $w_{q} \in[0,1]$ is the averaging weight, and $n$ represents discrete sampling with each packet arrival. The average queue-size is 
compared to two thresholds: the lower threshold $t h_{\min }$ and the upper threshold $t h_{\max }$. No packets are dropped when the average queue-size is below $t h_{\min }$. Between the lower and upper thresholds packets are dropped with an initial probability $p_{b}$, which is a function of $q_{a v e}$. As the average queue-size move between $t h_{\max }$ and $2 \cdot t h_{\max }$ the packets dropping probability $p_{b}$ grows linearly between $p_{\max }$ and 1 (RED gentle mode [7]).

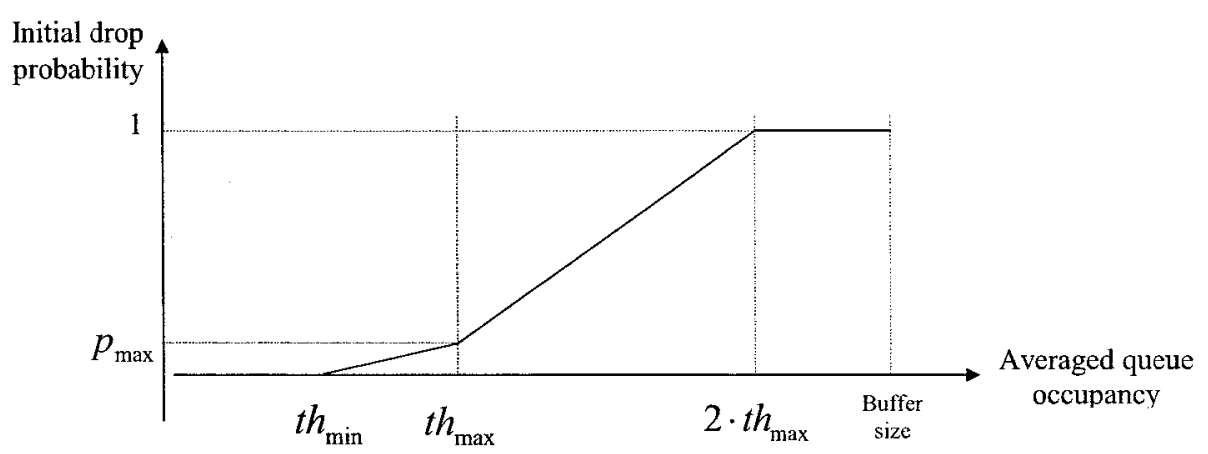

Fig. 2.3 RED Control Function

The relation between $P_{b}$ and $q_{\text {ave }}$ is formally defined by the following:

$$
p_{b}=\left\{\begin{array}{lr}
0, & q_{a v e} \leq t h_{\min } \\
a_{1}+b_{1} \cdot q_{a v e}, & t h_{\min }<q_{a v e} \leq t h_{\max } \\
a_{2}+b_{2} \cdot q_{a v e}, & t h_{\min }<q_{a v e} \leq 2 \cdot t h_{\max } \\
1, & q_{\text {ave }}>2 \cdot t h_{\max }
\end{array}\right.
$$

where the $p_{b}$ corresponding parameters are:

$$
a_{1}=-\frac{p_{\max } \cdot t h_{\max }}{t h_{\max }-t h_{\min }} \text {, and } b_{1}=\frac{p_{\max }}{t h_{\max }-t h_{\min }}
$$

for the area between the lower and upper thresholds in Fig. 2.3, and

$$
a_{2}=2 \cdot p_{\max }-1, \text { and } b_{2}=\frac{1-p_{\max }}{t h_{\max }}
$$

The final dropping probability is calculated using count of packets since the last drop:

$$
p_{a}=p_{b 1,2} /\left(1-\text { count } \times p_{b 1,2}\right)
$$


in-order to achieve a uniformly distribution and thus minimize the likelihood of bursts of consecutive drops. Let $\mathrm{X}$ be the number of packets arrivals from the last dropped packets (exclusive) to the next dropped packet (inclusive), and assuming a fixed average queuesize of $1 / p_{b 1,2}$ packets, then $\operatorname{Pr} o b[X=n]=p_{b 1,2}$ for $n \in\left[1,1 / p_{b 1,2}\right]$ and the mean value:

$$
E[X]=1 /\left(2 \cdot p_{b 1,2}\right)+0.5
$$

Several RED weaknesses were recognized (e.g., [11][14]) at the form of average delay and throughput sensitivity to the level of congestion and the parameter setting, which makes the queue behavior unpredictable a-priori. An Adaptive-RED was therefore devised [10]. Adaptive-RED is designed to automatically tune $p_{\max }$ to achieve target occupancy $q_{\operatorname{trg}}$ within $[0.4,0.6] \cdot\left(t h_{\max }-t h_{\min }\right) / 2$. The suggested Adaptive-RED further provides recommendation for setting $w_{q}$ as:

$$
w_{q}=1-\exp (-1 /(N \times R T T \times C))
$$

where $C$ is the link capacity in [packets/second], and the averaging-factor $N$ is the desired number of RTTs to average the queue-size to $63 \%$ of its instantaneous queue-size [10]. The target queue-size is set as a function of $t h_{\min }$ and $t h_{\max }$ levels:

$$
q_{\text {trg }}=\left(t h_{\max }+t h_{\min }\right) / 2
$$

with the recommended setting for $t h_{\max }$ as:

$$
t h_{\max }=3 \cdot t h_{\min }
$$

The controls of Adaptive-RED are summarized in Table 2.1:

\begin{tabular}{|l|l|}
\hline$B_{\text {len }}$ & Buffer length \\
\hline$w_{q}$ & Queue-size average weight \\
\hline$t_{\text {ratio }}$ & The ratio between max and min thresholds \\
\hline$q_{\text {rrg }}$ & The target queue fill during congestion \\
\hline
\end{tabular}

Table 2.1 Adaptive-RED Controls 


\section{Simulations Setup}

This section describes the simulation related setup and procedures common for the different chapters in this thesis.

\subsection{CDMA-2000 Reference Model}

The reference model of the wireless cellular system for the thesis focuses on the datanetwork interconnections. The involved components are the mobile-stations (MSs) that represent the data sources and sinks, the radio access network (RAN) that facilitates the transfer of the data over the wireless medium up to the Internet gateway, the Internet gateway Packet Data Service Node (PDSN), the Internet, and the Internet hosts. In the thesis we consider a single sided traffic, where the data flows from the Internet hosts towards the mobile nodes (forward link), and the ACKs flow in reverse link from the mobile hosts back to the Internet hosts (for the reliable TCP transport-layer traffic).

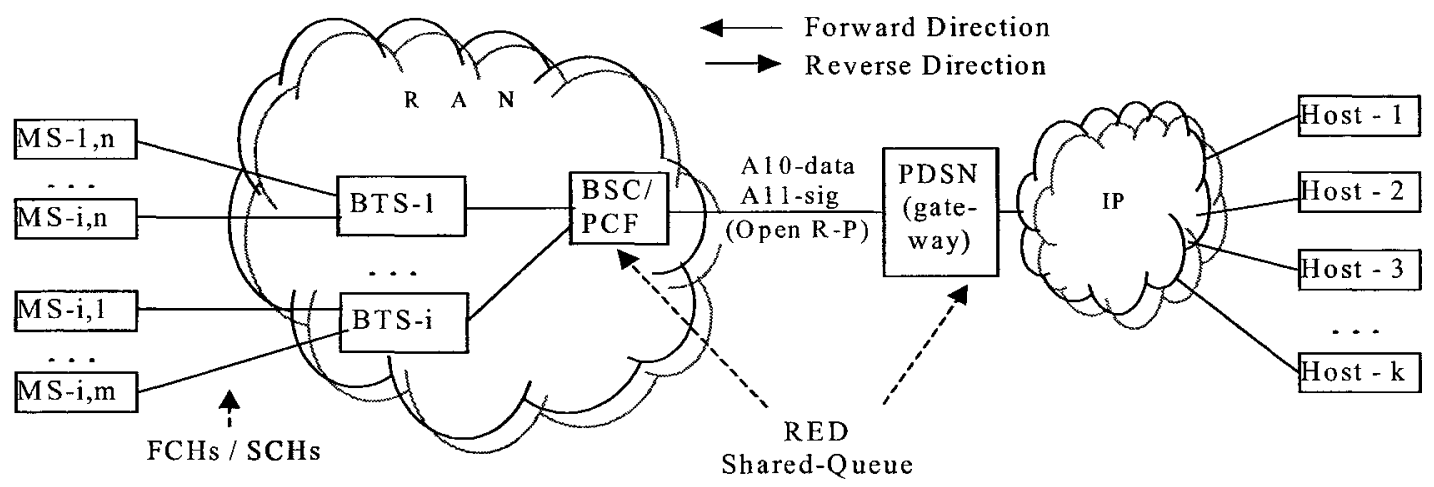

Fig. 3.1 CDMA-2000 Data-System Reference Model

\subsection{Traffic Sources}

This section decsribes the default trsffic setting. Specific test scenario may vary this setting. The simulations at the thesis selectively use four traffic source models over two transport layers. We do not claim to closely model the Internet traffic, but rather to provide a comprehensive performance evaluation and demonstrate the robustness of our proposed solution with various sources' scenarios and levels of burstiness. 
Two transport layers are used at the simulations: TCP and UDP. TCP Reno is used in the simulations with MSS of 576 bytes and maximum congestion window size of 10 packets for all flows. The CWND of all TCP source of $10 \mathrm{pkts} * 576 \mathrm{Bytes}$ is large such that it allows the system to limit the sources' rates. The TCP main parameters are summarized at Table 3.1. The UDP connections transmit packets with size of 576Bytes each.

The TCP connections may carry persistent sources, as well as interactive sources. The less bursty traffic is represented by persistent source in the form of infinite backlogged FTP source. The interactive traffic sources model generates the more bursty traffic at the form of a finite FTP with 'thinking' intervals. In the interactive traffic model the source sends files of fixed size, and after a file is transmitted successfully the source waits for a random period of time, uniformly distributed between 0 and twice the mean RTT (calculated excluding queuing delays), before transmitting the next file. This interactive source type resembles Web traffic while allowing us to focus on the data packets flow in the CDMA-2000 forward direction. All TCP sources are started randomly between 0 and $10 *$ RTT, with a uniform distribution.

The UDP connection may carry CBR or EXP application. A CBR application over the UDP is used with uniformly distributed random inter-packets interval over $\left[-0.5^{*} 1 /\right.$ rate, $+0.5 * 1 /$ rate], with a default mean rate that equals the 'TCP-friendly' rate [9]. We calculate the TCP-friendly rate as the bottleneck service rate divided by the total number of connections. The EXP application over UDP is set with an exponentially distributed source with $\mathrm{ON}: \mathrm{OFF}$ intervals. The mean values for the interval are $50 \mathrm{msec}: 100 \mathrm{msec}$, respectively. The rate during the $\mathrm{ON}$ interval has a mean value of double the TCPfriendly rate. Thus, the UDP rate is bursty, but on the average its rate is considered TCPfriendly. 


\begin{tabular}{|c|c|}
\hline Parameter & Value \\
\hline Version & Reno \\
\hline MSS & 576 \\
\hline $\begin{array}{c}\text { Maximum congestion- } \\
\text { window }\end{array}$ & 10 [packets] \\
\hline $\begin{array}{c}\text { Minimum RTO } \\
\text { Initial RTX }\end{array}$ & 0.4 \\
\hline TCP Segments per ACK & 3.0 \\
\hline
\end{tabular}

Table 3.1 TCP Transport Layer Setup

\subsection{System Setup}

This section describes the default system setups. These setups are uses in the proceeding system performances evaluation scenarios. In the various tests we may vary one or more of the setup's parameters from their default values.

The default CDMA-2000 system is constructed with two shared queues, one at the PCF and the other at the PDSN. 100 Internet-hosts communicating with 100 mobile stations, each using a unicast single-sided connection with traffic flowing from the Internet-hosts towards the mobile-stations at the forward direction (see Fig. 3.1). Each of the 100 active flows uses an RTT of $100 \mathrm{msec}$. The PCF to and from PDSN propagation delay is set to 5 [msec], which represents a backpressure close-loop delay of $10 \%$ of a flow's RTT (excluding queuing delays). The PCF shared-queue and the PDSN per class queues are constructed with RED AQM instance each. The PCF shared queue is constructed with service rate of $8 \mathrm{Mbps}$, whereas the PDSN queue with the superior characteristics is set with a multiplication factor of 3 to have a service-rate of $24 \mathrm{Mbps}$.

The target queue delay during congestion is set as a fraction of the end-to-end roundtrip time, and equals $0.5 \cdot R T T$ for both the PCF and PDSN queues. Consecutively, the PCF queue target queue occupancy during congestion is set to $0.5 \cdot R T T \cdot r_{P C F}$, where $r_{P C F}$ is the PCF service rate. The RED queues are constructed with buffer sizes to equal twice the specific RED instance's upper threshold. Consecutively, the buffer at the PCF 
shared queue equals $150 \mathrm{KByte}$, and the buffer at the PDSN shared-queue equals $450 \mathrm{KByte}$. The ratio between RED upper threshold and lower threshold is set to 3 . The queue averaging-weight $w_{q}$ is set to average the queue to $63 \%$ of its new value in 10 RTT . These RED queues' default controls setting follow the recommendations at [6] and [10] for the wired-Internet with the 'adaptive' and 'gentle' modes of operation.

A fundamental channel (FCH) is initially assigned for each of the wireless links. A corresponding supplemental-channel ( $\mathrm{SCH})$ is periodically assigned to each of the users in addition to FCH during the burst interval of the finite-burst mode, and de-assigned for the delay-interval. The FCH rate is $9.6 \mathrm{Kbps}$ each, whereas the $\mathrm{SCH}$ rate multiplication (multiplications of FCH) is 16 for each connection carrying infinite backlogged FTP, and 32 for each connection carrying interactive finite FTP traffic to allow greater traffic burstiness of the interactive finite FTP. The delay and burst intervals of the wireless links scheduling are 10 and $5.12 \mathrm{Sec}$, respectively. The rise and fall intervals are set to $6 \%$ of the 'burst' and 'delay' intervals, respectively. Each simulation's iteration lasts 200 seconds.

\subsection{System and Queuing Performance Metrics of Interest}

We characterize and evaluate the various flow-controls (RED, backpressure) and QoS mechanisms in the context of the CDMA-2000 as a function of temporal behavior, queuing system metrics, as well as backpressure specific metrics of interest. The term 'tandem queues' defines the pair of nodes, which are constructed of the PDSN as the feeding node and the PCF as the bottleneck. We define 'congestion period at the tandem queues' to be the time for which the aggregated wireless links rate is higher than the PCF service rate. This time interval represent potentially overloading inflow at the tandem queues ingress in which periods the PCF is the system bottleneck and is accordingly congested, and the PDSN is possibly congested subject to backpressure feedback indications. Measured metrics that require a mean of normalized values during 
congestion times utilize this 'congestion period' definition. We are interested in how the aggregate of multiple flows is affected by the flow-control and QoS mechanisms, rather than per flow performance.

The queuing system metrics of interest are as follows. In the context of the above definitions, throughput defines data throughput of the traffic aggregate as the bandwidth delivered to all receivers, excluding TCP duplicate packets and TCP or UDP traffic headers overhead. Normalized throughput values are normalizes each throughput reading with the sampled bottleneck bandwidth during congestion period at the PCF. Since the system bottleneck shifts between the link-queues and the PCF shared-queue during the burst interval, the sampled system bottleneck bandwidth may represent the PCF service rate, the PDSN service rate, or the wireless-links' aggregated service-rate, whichever is the lowest. This normalization aim to present the achieved throughput out of the system offered bandwidth (i.e., maximum achievable bandwidth) in the face of wireless links rate change. Delay examines the delay that packets experience while traversing a queue at the PCF, at the PDSN, or the tandem-queues, normalizes with the connections' mean RTT. Jitter measures the maximum variation in the PCF's shared-queue during congestion times, normalize with the connections' mean RTT, which is upper bounded by the queue's buffer-size. Data packet drop percentage is defined as the ratio between the drop packets volume to the volume of the total packets arriving at specific queue (at the PCF or the PDSN) throughout the simulation, presented as percentage. Overflow-based packet drops represent the volume of data packets dropped due to excessive congestion resulting with buffer overflowing condition, which is a portion of the Data packet drop metric.

The backpressure metrics of specific interest are as follows. Dedicated control packets represent the volume of control packets required for the backpressure mechanism to combat overflow events at the PCF queue or to control and stabilize the PCF. Control packets percentage is defined as the ratio between the dedicated control packets 
originated from the PCF towards the PDSN and the packets arrival volume at the PCF from the PDSN, in percentage. 


\section{Theoretical Analysis of RED in CDMA-2000}

A common mode of transmission in CDMA-2000, the 'finite-burst' mode, in which a supplemental channel ( $\mathrm{SCH})$ is periodically assigned for a user in addition to the already assigned fundamental channel (FCH), causes occupancy oscillations at the router's shared-queue and large overflow-based closely-clustered bursts of data-packets drops.

We formally investigate RED as a function of its controls' tuning space. The queuing transient response is derived using fluid-flow approximation with multi-sessions flowing through a shared queue. An estimate on the overflow-based drop burst volume at a RED shared queue is devised. Numerical solutions are provided and corresponding simulation results are presented as a function of variable RED controls, the CDMA-2000 system parameter-set, and the TCP connections' parameters.

The questions that this chapter targets are as follows. It questions whether a quantitative model of how to set the RED queue parameters as a function of the system parameters to avoid excessive spillover-based drops at the bottleneck queue can be derived for CDMA-2000 systems. It pursues the volume of the burst of overflow-based packet drop volume resulting from the wireless links' finite burst mode scheduling as a function of the RED controls and system parameters. It then questions: what are the preferred RED configurations for which the calculated losses-volume estimator is small such that it potentially allows the TCP senders to avoid the costly RTO based recovery? And, what are the setting-ranges for the RED controls to be avoided, which are characterized with excessive packets loss volume? Given the results space, it lastly questions the applicability of the RED mechanism to handle congestion at a CDMA-2000 shared-node.

We show that an overflow-based excessive packet discard volume is unavoidable with certain RED controls setting ranges. We show that the current literature recommendations for RED setting are inadequate for handling the finite-burst mode. We use the model to specify the preferred tuning areas for RED with low overflow-based drops. 


\subsection{Analysis of the CDMA-2000 Model}

The reference model of the wireless cellular system for the analysis is as depicted by Fig. 3.1. The system assumptions follow section 3. In particular, A fluid of $N$ long-lived persistent TCP connections with equal RTT operate in their congestion avoidance stage. The network has a single fixed service-rate bottleneck at the PCF. A unidirectional traffic at the forward direction is analyzed. RED concepts and controls are described at 2.2.

\subsubsection{Queuing Model}

Fig. 4.1 shows the referenced queuing model. The link buffers represent the radio-links' buffers. The service-rate for each link buffer is switched between $\eta_{l l}$ that corresponds to the base $\mathrm{FCH}$ rate, and $\eta_{l h}$ that equals the $\mathrm{FCH}$ and $\mathrm{SCH}$ aggregated rate. The finite shared-buffer available space and the service-rate are denoted by $B_{S}$ and $\eta_{S}$, respectively. We concentrate on the case where $N \cdot \eta_{l l} \leq \eta_{S} \leq N \cdot \eta_{l h}$ in which the queued workloads oscillate between shared-buffer and link-buffers as the links rates fluctuate. The link buffers' aggregated occupancy and the shared buffer's occupancy are denoted as $q_{i}^{L}(t)$ and $q_{i}^{S}(t)$, respectively, and $T$ is the round-trip propagation delay lumped between each source and sink.

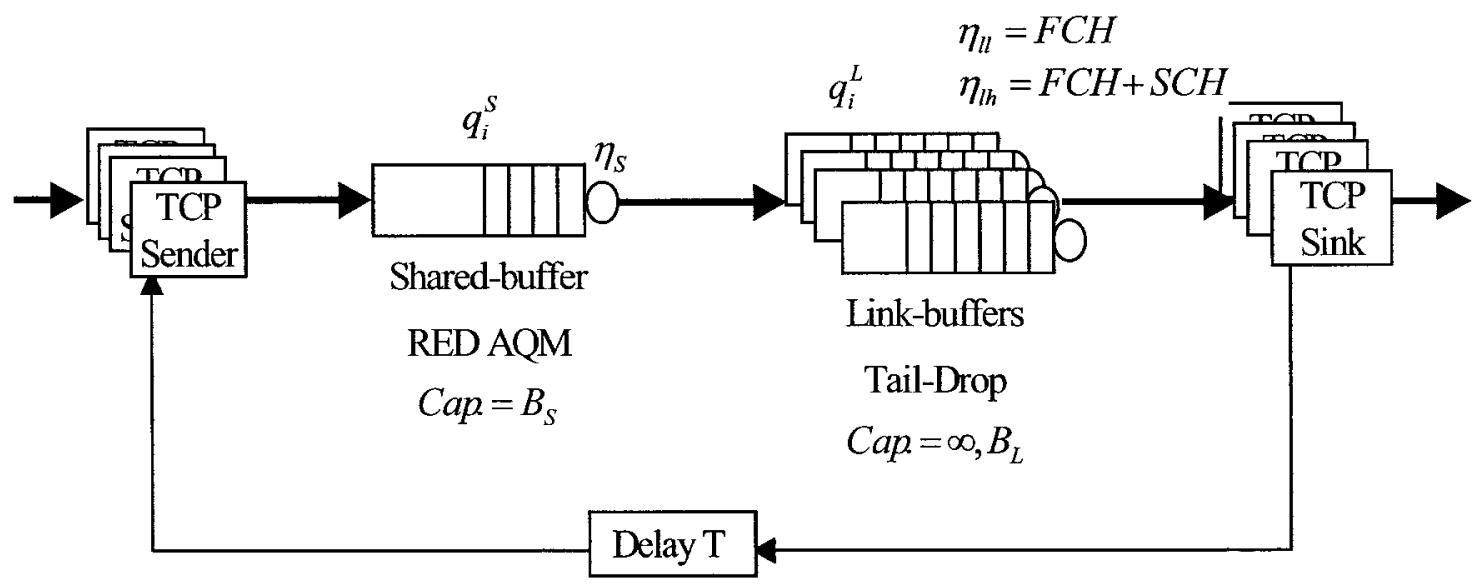

Fig. 4.1 Queuing Model for Packet-Losses at the Shared-Buffer 


\subsubsection{System Dynamics Analysis}

We analyze the impact of a single link-rate switching occurring simultaneously at all the wireless links. Let assume that $N$ homogeneous mobiles were operating each with a link rate of $\eta_{l l}$ for a long duration prior to time $t_{0}$ such that for each a steady-state TCP congestion window size of $W$ has reached. The shared-buffer service-rate is greater than the link-queues aggregated service-rate, thus the TCP traffic is shaped to the link-queues lower rates and the content of shared-buffer is $q_{0}^{s}(t)=0$. Consecutively, the content at each link-buffer is $q_{0}^{L}(t)=W-\eta_{l l} \cdot T$. At the instance $t_{0}$ each link's rate is switched to the higher value of $\eta_{l h}$, resulting with faster packet arrivals at the TCP sinks sourced from the link-buffer, faster generation of ACKs, and more traffic injected towards the shared-queue by the TCP senders. Thus, at this instance the queued workloads in link buffers begin to move towards the shared buffer.

The starting time instance and the ending instance of the queued workload transfer, during which the shared buffer begins to fill and possibly overflows, are of interest in the model analysis. The start time, when leading edge of packets at higher rate reaches the shared buffer, is $t_{\text {start }}=t_{0}+T$ since it takes a round-trip time for both the ACKs and the consequent data-packets to flow through the system. The end time instance $t_{\text {end }}$, when the high-rate packets flow stops at the shared-queue ingress, takes one of the following three possible cases.

In the $1^{\text {st }}$ case for $t_{\text {end }}$, the leading edge of packets fluid-flow at higher rate reaches the shared-buffer before the links buffers run empty, and the TCP senders become aware of a drop event in the shared-buffer before it stops receiving packets at higher rate. Let assume that when a TCP sender receives first trail of packet flow after a drop event, it stops sending packets at higher rate irrespective of ACK arrivals rate. In practice, it waits till it gets three duplicate ACKs, after which it retransmits the lost segment and halves its congestion window. Table 1 shows the time instances and the queues occupancies for 
each of the ruling events at the fluid-flow analysis for this case. Each of the link-buffers begins to empty at $t>t_{1}$ with a rate $d q^{L}(t) / d t=\eta_{l h}-\eta_{l l}$, and the shared-buffer begins to fill at $t>t_{2}$ with a rate $d q^{S}(t) / d t=N \cdot \eta_{l h}-\eta_{S}$. Let's denote $\mathrm{q}_{\mathrm{EDI}}^{\mathrm{s}}$ as the instantaneous shared-queue occupancy at the time-instance in which the first probabilistic early-drop occurs. It is upper-bounded by the buffer-size. Then, the first probabilistic early-drop at the shared-queue occurs after another $\min \left(\mathrm{q}_{\mathrm{ED} 1}^{\mathrm{s}}, \mathrm{Bs}\right) /\left(N \cdot \eta_{l h}-\eta_{S}\right)$ seconds. It takes additional $\min \left(q_{E D 1}^{\mathrm{S}}, \mathrm{Bs}\right) / \eta_{s}$ seconds for the leading edge of the trail behind first early-drop to leave the shared buffer. It takes this trail $q_{4}^{L} / \eta_{\text {th }}$ seconds to traverse a link-queue, an interval that represents the draining period of the remaining content in a particular linkqueue ahead of the trail. After additional $T$ seconds the first TCP sender senses a lost segment and stops further high-rate transmission. At this time, the shared-queue egress rate start to decrease till it potentially falls below $\eta_{S}$ as sufficient number of TCP senders sense subsequent probabilistic early-drops.

An estimate on the packet loss-value can be obtained by calculating the packets volume surplus at $t_{\text {end }}$ with respect to the shared-queue capacity; that is, $q_{6}^{S}-B_{S}$. The estimate represents a lower bound on the overflow-based packet-losses at the sharedqueue since a subsequent transient period in which the aggregated packet flow-rate decreases from $N \cdot \eta_{l h}$ to $\eta_{S}$ is anticipated. The governing condition for this case is obtained by condition the links queues to have sustainable workload throughout the above chain of events till the first TCP sender senses the early-drop indication; that is, $q_{5}^{L} \geq 0$. This leads to the condition $\eta_{l h} \leq W /\left(T+B_{S} / \eta_{S}\right)$. The estimate on the lossvolume at $t_{\text {end }}$ can therefore be written:

$L_{V}^{I} \geq \max \left(W \cdot\left(N-\frac{\eta_{S}}{\eta_{l h}}\right)+\min \left(q_{E D 1}^{S}, B_{S}\right)-L_{V}^{E D}-B_{S}, 0\right) \quad$ if $\quad \eta_{l h} \leq \frac{W}{T+\min \left(q_{E D 1}^{S}, B_{S}\right) / \eta_{S}}$ 
where $L_{V}^{E D}$ is early drops loss-volume before the queue overflows, for which a mathematical expression is derived at section 4.1.3.2. The early drops loss-volume is subtracted from the volume of the overflow drops since we are interested in evaluating the burst of clustered drops rather than estimating the packets that are properly dropped by the RED mechanism in a distributed and random manner.

\begin{tabular}{|c|c|c|c|c|}
\hline$i$ & $\begin{array}{c}\text { Time } \\
\left(t_{i}=t_{i-1}+\Delta t_{i}\right)\end{array}$ & $\begin{array}{c}\text { Link-queues' } \\
\text { aggregated } \\
\text { occupancy } q_{i}^{L}(t)\end{array}$ & $\begin{array}{c}\text { Shared-queue } \\
\text { instantaneous } \\
\text { occupancy } q_{i}^{S}(t)\end{array}$ & Event \\
\hline 1 & $t_{0}$ & $N \cdot\left(\overline{\left.W-\eta_{l l} \cdot T\right)}\right.$ & 0 & Steady-State \\
\hline 2 & $t_{1}+T$ & $q_{1}^{L}-\Delta_{2} \cdot N \cdot\left(\eta_{l h}-\eta_{l l}\right)$ & 0 & $\begin{array}{l}\text { High-rate flow leading edge } \\
\text { reaches shared-queue }\end{array}$ \\
\hline 3 & $t_{2}+\frac{q_{3}^{S}}{N \cdot \eta_{l h}-\eta_{S}}$ & $q_{2}^{L}-\Delta_{3} \cdot\left(N \cdot \eta_{l h}-\eta_{S}\right)$ & $\min \left(q_{\mathrm{ED} 1}^{\mathrm{s}}, \mathrm{Bs}\right)$ & $\begin{array}{l}\text { First early-drop at shared- } \\
\text { queue }\end{array}$ \\
\hline 4 & $t_{3}+\frac{q_{3}^{s}}{\eta_{s}}$ & $q_{3}^{L}-\Delta_{4} \cdot\left(N \cdot \eta_{l h}-\eta_{S}\right)$ & $q_{3}^{S}+\Delta_{4} \cdot\left(N \cdot \eta_{l h}-\eta_{S}\right)$ & $\begin{array}{l}\text { Trail behind first early-drop } \\
\text { leaves the shared-queue }\end{array}$ \\
\hline 5 & $t_{4}+\frac{q_{4}^{L} / N}{\eta_{l h}}$ & $q_{4}^{L}-\Delta_{5} \cdot\left(N \cdot \eta_{i h}-\eta_{S}\right)$ & $q_{4}^{S}+\Delta t_{5} \cdot\left(N \cdot \eta_{l h}-\eta_{S}\right)$ & $\begin{array}{l}\text { Trail behind first early-drop } \\
\text { leaves a link-queue }\end{array}$ \\
\hline 6 & $t_{5}+T=t_{\text {end }}^{I}$ & & $q_{5}^{S}+\Delta_{6} \cdot\left(N \cdot \eta_{l h}-\eta_{S}\right)$ & $\begin{array}{l}\text { High-rate flow start } \\
\text { decreasing at the shared- } \\
\text { queue's ingress }\end{array}$ \\
\hline
\end{tabular}

Table 4.1 Continuous fluid-flow Analysis for the $1^{\text {st }}$ case

A couple of specific cases in the system dynamics for $t_{\text {end }}$ and $L_{V}$ are as follows. In the first case, denoted as the $2^{\text {nd }}$ case for $t_{\text {end }}$ and $L_{V}$, the link buffers run empty before the leading edge of packet flow at higher rate reaches shared buffer. In the second case, denoted as the $3^{\text {rd }}$ case for $t_{\text {end }}$ and $L_{V}$, the leading edge of packet flow at higher rate does reaches the shared buffer before the links buffers run empty, however the TCP senders stop receiving packets at a higher-rate before they become aware of early-drops 
events in shared buffer. Once the link-queues are emptied, they resume the transmission upon packets arrivals at rate $\eta_{S}$. An early-drop at a RED queue is intended to discourage overflow through implicit congestion indications to the TCP senders, rather than physically throttling down the traffic. Even though early drops as well as overflow are possible for these two cases at the shared queue, an analysis of the model with earlydrops for these case is of minor interest since the TCP senders stop sending packets at higher rate due to the cease of arrivals at high-rate rather than the sensing of an earlydrop indication, hence the effect of an early-drop is low. Therefore, the shared-buffer content can be closely approximated with the results at [4] obtained for a single flow with a tail-drop discipline at the shared-queue. Adapting the result to fluid-flow of $N$ TCPs, accounting for the possible early-drops, and considering a complete queue assigned for the $N$ connections, an estimate on the loss-volume at $t_{\text {end }}$ can be written as:

$$
\begin{gathered}
L_{V}{ }^{I I} \geq \max \left(\frac{\left(W-\eta_{l l} \cdot T\right) \cdot\left(N \cdot \eta_{l h}-\eta_{S}\right)}{\eta_{l h}-\eta_{l l}}-L_{V}^{E D}-B_{S}, 0\right) \text { if } \eta_{l h} \geq \frac{W}{T} \\
t_{\text {end }}^{I I}=\frac{W-\eta_{l l} \cdot T}{\eta_{l h}-\eta_{l l}}+T
\end{gathered}
$$

and:

$$
\begin{gathered}
L_{V}{ }^{I I I} \geq \max \left(N \cdot W-\eta_{S} \cdot T-L_{V}^{E D}-B_{S}, 0\right) \text { if } \frac{W}{T+\min \left(q_{E D !}^{S}, B_{S}\right) / \eta_{S}}<\eta_{l h} \leq \frac{W}{T} \\
t_{\text {end }}^{I I I}=\frac{N \cdot\left(W-T \cdot \eta_{l h}\right)}{N \cdot \eta_{l h}-\eta_{S}}+T
\end{gathered}
$$

\subsubsection{RED Dynamics Analysis at the Context of CDMA-2000}

The variables $q_{E D 1}^{S}$ and $L_{V}^{E D}$ for the equations above are derived in sections 4.1.3.1 and 4.1.3.2, respectively. 


\subsubsection{Dynamics Before Early-Drops $\left(q_{a v e} \leq t h_{\min }\right)$}

Using the discrete time Eq.(2.1) and Z-transform, RED low-pass filter's transfer function can be rewritten as:

$$
H_{\text {ave/inst }}(z)=\frac{q_{\text {ave }}(z)}{q_{\text {inst }}(z)}=\frac{z \cdot w_{q}}{z-\left(1-w_{q}\right)}
$$

The grow rate of instantaneous PCF shared queue size is a linear increasing function and depends on the packet arrival-rate and the packets departure rate before any packets blocking events occur. The case presents the time interval after the SCHs allocations, before the queue is overflowed, and before the average queue-size exceeds the packetdrops lower threshold. The traffic from multiple TCP senders is aggregated at the RED shared-buffers ingress, and approximated as a continuous fluid-flow. As aforementioned, the system bottleneck is shifted to the shared-queue once SCHs are allocated to the mobile-users. At this time instance the fluid at the shared-queue's ingress is $N \cdot \eta_{l h}$ [packets/Sec], whereas the instantaneous queue-size grows with a rate of $N \cdot \eta_{l h}-\eta_{S}$ [packets/Sec]. Thus the relationship between the shared-queue inflow rate and its instantaneous size growth-rate during this time interval is:

$$
h_{\text {inst } / \text { in }}=\frac{N \cdot \eta_{l h}-\eta_{S}}{N \cdot \eta_{l h}}=1-\frac{\eta_{S}}{N \cdot \eta_{l h}}
$$

We are interested in obtaining a relationship between the queue internal metrics; i.e., the average queue-size that is directly related to the starting of early-drops, and the queue instantaneous size that is directly related to the overflow event. The instantaneous queuesize can be written as a ratio of the ingress packets function $n \cdot U[n]$ as:

$$
q_{\text {inst }}[n]=h_{\text {inst } / \text { in }} \cdot n \cdot U[n]
$$

and in Z-plane:

$$
q_{\text {inst }}(z)=h_{\text {inst } / \text { in }} \frac{z}{(z-1)^{2}}
$$


The RED average queue-size with the fluid-flow ingress can be calculated using Eq.(4.6) and Eq.(4.9):

$$
q_{\text {ave }}[n]=\mathrm{Z}^{-1}\left\{H_{\text {ave / inst }}(z) * q_{\text {inst }}(z)\right\}=h_{\text {inst } / \text { in }} \cdot\left(n+1+\frac{\left(1-w_{q}\right)^{n+1}-1}{w_{q}}\right)
$$

where $n$ presents packet-arrival instances. Assigning the inverse of Eq.(4.8) (i.e., presenting the incoming number of packets as a function of the instantaneous queue-size) in Eq.(4.10) yields:

$$
q_{\text {ave }}\left[\frac{q_{\text {inst }}}{h_{\text {inst } / \text { in }}}\right]=q_{\text {inst }}+h_{\text {inst /in }} \cdot\left(1+\frac{\left(1-w_{q}\right)^{\left(g_{\text {inst }} / h_{\text {ins } / \text { int }}\right)+1}-1}{w_{q}}\right) \quad \forall \quad \frac{q_{\text {inst }}}{R_{\text {inst }}} \in \mathrm{N}^{*}
$$

where the $q_{\text {inst }} / h_{\text {inst } t \text { in }}$ is a natural number (excluding the zero) that equals the number of incoming packets.

The average queue size is required to exceed the lower-threshold for initiating the forwarding of congestion indications to the TCP senders via early packets drops; that is:

$$
q_{\text {ave }}\left[q_{\text {inst }} / h_{\text {inst } / \text { in }}\right]>t h_{\min }
$$

The lower threshold can be presented as a function of the RED control parameters.

Combining Eq.(2.8) with the parameterized form of Eq.(2.9) as $t h_{\max }=t h s_{\text {ratio }} \cdot t h_{\min }$, we obtain:

$$
t h_{\min }=\frac{2 \cdot q_{\text {trg }}}{1+t h s_{\text {ratio }}}
$$

where $q_{t r g}$ is in packets. On the other hand, the target queue-delay is recommended at [10] to be a fraction of the round-trip time, or equivalently the target queue-size to be a fraction of the idle (i.e., no queuing) pipe-capacity:

$$
q_{\text {trg }}=\eta_{S} \cdot \Phi_{\text {trg }} \cdot T
$$

where $\Phi_{\text {trg }}$ is the queue target ratio. Combining Eq. (4.13) and Eq.(4.14), the lower threshold can be rewritten as: 


$$
t h_{\min }=\frac{2 \cdot \eta_{S} \cdot \Phi_{t r g} \cdot T}{1+t h s_{\text {ratio }}}
$$

Assigning Eq.(4.11) and Eq.(4.15) at Eq.(4.12), the first early drop $q_{E D 1}^{s}$ is obtained from the inequality for the instantaneous queue occupancy as a function of the RED controls for the transient response starting from the SCHs allocations and till the first early drop:

$$
\left\{q_{E D 1}^{S}=\min \left(q_{\text {inst }}^{S}\right): q_{\text {inst }}^{S}+R_{\text {inst }} \cdot\left(1+\frac{\left(1-w_{q}\right)^{\frac{q_{\text {instlin }}^{S}}{h_{\text {ins }}}-1}-1}{w_{q}}\right)>\frac{2 \cdot \eta_{S} \cdot \Phi_{\text {trg }} \cdot T}{1+t h S_{\text {ratio }}} \quad \forall \frac{q_{\text {inst }}^{S}}{h_{\text {inst in }}} \in \mathrm{N}^{*}\right\}
$$

where the operand $\{\operatorname{expr} 1: \operatorname{expr} 2\}$ means that exprl is true such that expr2 is satisfied.

\subsubsection{Early-Drops Volume Analysis Prior to Queue Overflowing}

We are interested in evaluating the closely clustered overflow-based packets drops, whereas RED's probabilistic early drops prior to the overflow occurrence are considered as proper measurement in congestion handling. Hence, this section formulates an estimate on the volume of the early-drops prior to the buffer overflowing event.

It is desired to approximate the RED actual drop probability to be a function of $q_{\text {ave }}^{s}$ alone for incorporating in our continuous flow model. We denote the approximated drop probability as $p_{C F}$. Let $Y$ be the number of dropped packets in $n$ Binomial trials (i.e., packet arrivals). Requiring the expected value $E[Y]=n \cdot p_{C F}$ to be exactly one dropped packet in $n=E[X]$ (Eq.(2.6)) number of arrivals, then the estimated drop probability can be written as:

$$
p_{C F}[n]=\frac{2 \cdot p_{b 1,2}[n]}{1+p_{b 1,2}[n]}
$$

The ratio between the shared-queue inflow rate and its instantaneous size increase at Eq. (4.7) needs to be adjusted with the eventual early-drops during the time-interval where $\mathrm{th}_{\min }<\mathrm{q}_{\mathrm{ave}}^{\mathrm{s}}[\mathrm{n}] \leq 2 \cdot \mathrm{th}_{\max }$. The shared-queue ingress rate is reduced by the blocking 
rate and can be written as $N \cdot \eta_{l h} \cdot\left(1-p_{C F}\right)-\eta_{S}$, while the egress rate sustains the rate of $N \cdot \eta_{l h}$. Therefore, the ratio can be rewritten as:

$$
h_{i n s t / \text { in }}[n]=1-p_{C F}[n]-\frac{\eta_{S}}{N \cdot \eta_{l h}}
$$

The shared queue instantaneous size can now be expressed as a function of the TCP senders' aggregated rate and RED's blocking rate using the following recursive form:

$$
q_{\text {inst }}^{S}[n]=q_{\text {inst }}^{S}[n-1]+h_{\text {inst /in }}[n]
$$

The shared queue RED characteristics depicted by Eq.(2.1) and Eq.(4.19) introduce a complex relationship between $q_{\text {inst }}^{S}$ and $q_{\text {ave }}^{S}$ for $h_{\text {inst } / \text { in }}$ at the form of Eq.(4.18), therefore we use these equations at their $n$ domain (recursive form) for the early-drop estimate in our model.

A couple of initial conditions are required in order to solve Eq.(4.19) (for $\left.q_{i n s t}^{S}\right)$ and Eq.(2.1) (for $q_{a v e}^{s}$ ) in the range of early drops $\left(\mathrm{th}_{\min }<\mathrm{q}_{\mathrm{avc}}^{\mathrm{s}}[\mathrm{n}] \leq 2 \cdot \mathrm{th}_{\max }\right)$. We first define the discrete time instance for the initial conditions as a function of the number of arrivals $n$. Letting $n=0$ be $t_{2}$ at Table 4.1 (in which the leading edge of the high rate flow reaches the shared-queue ingress), then the number of packet arrivals $n_{\text {start }}$ for the initial conditions equals the number of packet arrivals just before the first possible early-drops, bounded by the number of packets to completely fill the queue:

$$
n_{\text {start }}=\min \left(n_{\text {th }},\left\lfloor B_{S} / h_{\text {inst } / \text { in }}\left(p_{C F}=0\right)\right\rfloor\right)
$$

where $n_{t h_{\min }}$ is defined as:

$$
\left\{n_{t h_{\min }}=\max (n): \quad h_{i n s / / i n}\left(p_{C F}=0\right) \cdot\left(n+1+\frac{\left(1-w_{q}\right)^{n+1}-1}{w_{q}}\right) \leq t h_{\min }, \quad \forall n \in \mathrm{N}\right\}
$$

which uses Eq.(4.10), and $t h_{\min }$ as described at Eq.(4.13). The initial conditions can now be obtained as: 


$$
q_{\text {ave }}^{S}\left[n_{\text {start }}\right]=h_{\text {inst /in }}\left(p_{C F}=0\right) \cdot\left(n_{\text {start }}+1+\frac{\left(1-w_{q}\right)^{n_{\text {sarr }}+1}-1}{w_{q}} \quad \forall n \in \mathrm{N}\right)
$$

and:

$$
q_{\text {inst }}^{S}\left[n_{\text {start }}\right]=n_{\text {start }} \cdot h_{\text {inst in }}\left(p_{C F}=0\right)
$$

where $h_{\text {inst } / \text { in }}$ is used with no drops form due to the absence of any drops while RED's average queue-size is below the RED's lower threshold.

Focusing on estimating the early drops before the first buffer overflow event, the time instance in which the clustered overflow-based drops begins is required. Expressed as discrete time samples of the RED queue, the number of arrivals at this instance equals:

$$
n_{\text {end }}=\min \left(n_{O F},\left(t_{\text {end }}^{I, I I, I I I}-T\right) \cdot N \cdot \eta_{l h}\right)
$$

where the right term bounds $n_{\text {end }}$ with the end time of the high-rate inflow into the shared-queue due to empty link-buffers or early-drop notification as described at Table 4.1 (last row), Eq.(4.3), or Eq.(4.5), subject to the governing condition on $\eta_{l h}$. The left term in the min function, which described the start of the overflow-based drops, can be written as:

$$
\left\{n_{O F}=\max (n): \quad q_{\text {inst }}^{S}[n] \leq B_{S} \quad \text { and } \quad q_{\text {ave }}^{S}[n] \leq 2 \cdot t h_{\max }\right\}
$$

We calculate the expected number of early drops at the prescribed time interval. Let $S$ be the number of dropped packets considering Bernoulli trials for each arrival. Using the continuous flow approach we considering fractions of packet discards as well, and the expected value for each arrival is $E[S]=1 \cdot p_{b 1,2}+0 \cdot\left(1-p_{b 1,2}\right)$. Summing the packet loss for all $q_{a v e}^{s}[n]$ between $n_{\text {start }}$ and $n_{\text {end }}$, the early-drops packet losses volume before the buffer overflow event therefore can be written as:

$$
L_{V}^{E D}=\sum_{n_{\text {start }}+1}^{n_{\text {aqd }}}\left\{\begin{array}{lll}
p_{C F 1}\left(q_{\text {ave }}^{s}[n]\right), & \text { if } & t h_{\min }<q_{\text {ave }}^{s}[n] \leq t h_{\max } \\
p_{C F 2}\left(q_{\text {ave }}^{s}[n]\right), & \text { if } & t h_{\max }<q_{\text {ave }}^{s}[n] \leq 2 \cdot t h_{\max }
\end{array}\right.
$$


Note that floor value, as preset by RED adaptive mechanism, is assumed for the adaptive RED's parameter $p_{\max }$ at Eq.(2.3) and Eq.(2.4) due to the empty shared-queue prior to the SCHs allocations at the related bottleneck shift. Furthermore, $p_{\max }$ is assumed to be fix till the time instance $n_{\text {end }}$ under the assumption of either an adaptation interval of $p_{\max }$ larger than the elapsed time interval between $t_{2}$ at Table 4.1 and the $n_{\text {end }}$, or a negligible adaptation value.

\subsubsection{Early-Drops Volume Analysis During Queue Overflowing}

It is desired to compare our model with simulation results obtained with equivalent system. The operation of the queue averaging and the early-drop mechanisms persist during overflowing periods in RED implementation at NS2 [33]. This section deduces an estimate on the counted early-drops in RED implementations during the captured overflow time interval by our model due to packet departures from the shared queue amid consecutive overflow-based drops. We use it to decrease our overflow-based drops estimate $L_{V}$ stated at Eq.(4.1), Eq.(4.2), and Eq.(4.4).

Lets denote $R_{\text {OFdrop }}^{\text {measure }}$ as the estimate on the measured overflow-based drop rate (or equivalently the fluid-flow overflow-based measured dropped portion of each arrival) during buffer overflow interval. The expected value of the fluid-flow measured randomdrop for each arrival's remainder after the overflow-based drops effect during buffer overflow using Bernoulli distribution is $\left(1-R_{\text {OFdrop }}^{\text {measure }}[n]\right) \cdot p_{C F}[n]+0 \cdot\left(1-p_{C F}[n]\right)$. The sum of the two equals the theoretical overflow-based drop volume for each arrival at Eq.

$$
R_{\text {OFdrop }}^{\text {measure }}[n]+p_{C F}[n] \cdot\left(1-R_{O F \text { drop }}^{\text {measure }}[n]\right)=h_{\text {inst } / \text { in }}\left(p_{C F}=0\right)
$$

since on the average the queue equals its buffer size Bs during the overflow interval, which means that the total drop rate (the left term) equals the queue instantaneous fill potential grow rate without drops (the right term). 
The early-drop portion during the overflow period for each packet arrival (and equivalently, the dropping rate) can be written as: $R_{\text {edrop }}^{\text {measur }}[n]=h_{\text {inst } / \text { in }}\left(p_{C F}=0\right)-R_{\text {OFdrop }}^{\text {measur }}[n]$. Solving (4.27) for $R_{O F d r o p}^{\text {measure }}[n]$, and assign at $R_{\text {edrop }}^{\text {measure }}[n]$ we get:

$$
R_{\text {edrop }}^{\text {measure }}[n]=p_{C F 1,2}[n] \cdot \frac{1-h_{\text {inst } / \text { in }}\left(p_{C F}=0\right)}{1-p_{C F 1,2}[n]}
$$

and the early-drops volume throughout the overflow period, using the expected-value for each arrival as a Bernoulli trial as the discussed at section 4.1.3.2, can be written as:

$$
L_{V}^{E D @ O F} \geq \sum_{n=n_{O F}+1}^{n_{\text {end }}^{E D O F}}\left\{\begin{array}{cl}
p_{C F 1,2}[n] \cdot \frac{1-h_{\text {inst } t \text { in }}\left(p_{C F}=0\right)}{1-p_{C F 1,2}[n]} & \text {, if } t h_{\min }<q_{a v e}[n] \leq t h_{\max } \\
p_{C F 1,2}[n] \cdot \frac{1-h_{\text {inst } / \text { in }}\left(p_{C F}=0\right)}{1-p_{C F 1,2}[n]} & \text { if } t h_{\max }<q_{a v e}[n] \leq 2 \cdot t h_{\max } \\
0 & , \text { elsewhere }
\end{array}\right.
$$

where, $n_{O F}$ is given by Eq.(4.25), $n_{\text {end }}^{E D @ O F}=\left(t_{\text {end }}^{I, I I, I I I}-T\right) \cdot N \cdot \mu_{l h}$, and $t_{\text {end }}^{I, I I I I}$ is given by Eq.(4.3), Eq.(4.5), or Table 4.1, according to the governing condition on $\mu_{l h}$ there. For calculating $p_{C F 1,2}\left(q_{a v e}^{S}[n]\right)$, the instantaneous queue-fill $q_{\text {inst }}^{s}$ simply equals the buffersize $B_{S}$. Then the average queue-size using Eq. (2.1) equals $q_{a v}[n+1]=\left(1-w_{q}\right) \cdot q_{a v}[n]+w_{q} \cdot B_{S}$ and $p_{C F 1,2}[n]$ follows Eq.(4.17). All drops occurring during periods for which $q_{a v e}[n]>2 \cdot t h_{\max }$ are considered at the RED implementation as "forced" drops, hence no early-drops being counted at the $L_{V}^{E D @ O F}$ estimate. The two initial conditions $q_{\text {inst }}^{S}\left[n_{O F}\right]$ and $q_{\text {iave }}^{S}\left[n_{O F}\right]$ follow Eq.(4.22) and Eq.(4.23) by substituting $n_{\text {start }}$ with $n_{O F}$ for $n_{O F} \leq n_{t h_{\min }}$ (since there are no early-drops prior to $n_{O F}$ time instance), or equal the values of $q_{\text {inst }}^{S}\left[n_{\text {end }}\right]$ and $q_{\text {iave }}^{S}\left[n_{\text {end }}\right]$ as at the exit point of Eq.(4.26) recursion for $n_{O F}>n_{t h_{\min }}$. Lastly, the modified estimate for the purpose of the study-case and the pertaining numerical results validation with simulations is: 


$$
L_{V}^{\text {measure }}=L_{V}-L_{V}^{E D @ O F}
$$

where $L_{V}$, our theoretical overflow estimates are given by Eq.(4.1), Eq.(4.2), and Eq.(4.4), subject to their governing condition.

Note the inequality in Eq.(4.29). In practice, the early-drops effect during overflow intervals can be greater than $L_{V}^{E D @ O F}$ estimator's equality expression. Traffic burstiness may cause larger early-drops amid overflow due to small occupancy variation close to the buffer overflow condition. Such variations are not capture by our model, but were observed at system simulations.

\subsection{Application to CDMA-2000 System Parameters}

This section provides numerical solutions to the estimate on the overflow-based drops burst volume with varied RED controls to demonstrate the CDMA-2000 system behavior with a RED shared-queue. It further validates the numerical solutions with simulation results of an equivalent system.

The defaults system and traffic setup for this case study follows the defaults in chapter 3 with the following variation and additions. The target queue size during congestion is set as a fraction of the end-to-end round-trip time, and equals $0.75 \cdot R T T$. The queue buffer-size is set to 2.25 the link capacity (equals 3 times the average queue-size) in packets. The system carries 100 active flows, each using an infinite backlogged TCP connection. The steady-state TCP congestion-window reached before the FCH allocation for each of the connections is 10 packets. Each of the parameters is being varied from its initial setting to demonstrate its effect on the losses-volume estimate. The FCHs are concurrently allocated with finite-burst mode scheduling 'burst' interval of 2.56 seconds each [2][3].

The numerical results of the $L_{V}^{\text {measure }}$ estimator with comparison to the captured overflow-based drops from simulations are presented at Fig. 4.2. The measurements were performed using the NS-2 simulator. Our estimated overflow-based losses-volume shows 
to closely approximate the simulation results. The estimate $L v$ of the total overflow losses-volume (Eq.(4.1),(4.2),(4.4)) calls for losses-volume possibly larger than the indicated equality since in practice multiple drops are required to lower the shared-queue ingress rate to below its egress rate. On the other hand, the estimate for the early-drops losses-volume $L_{V}^{E D @ O F}$ during the overflow interval at Eq.(4.30), which is reduced from $L_{V}$ to obtain the measured overflow-based losses-volume, as explained earlier calls for possibly larger early-drops volume than its indicated equality. The combination of these two estimates at Eq.(4.30), for our tested parameters' ranges as presented Fig. 4.2 graphs, show to closely compensate each other.

(a)

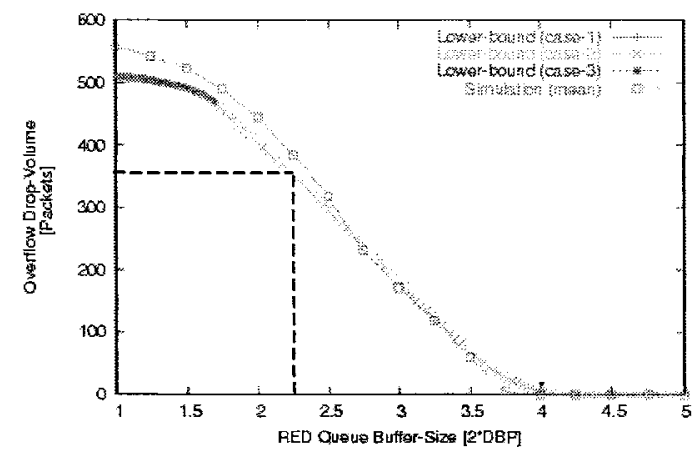

(c)

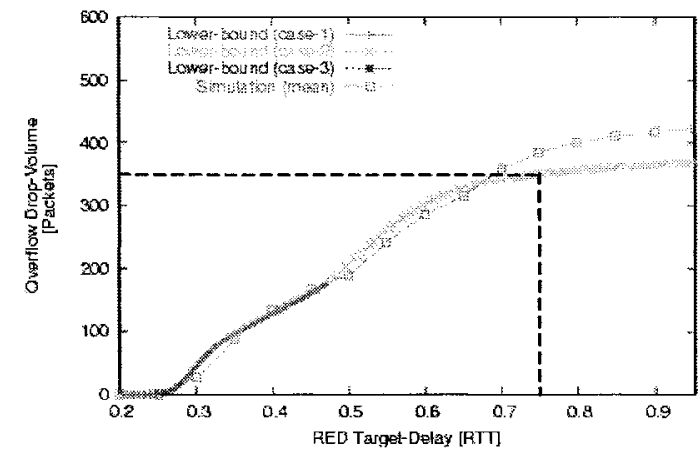

(b)

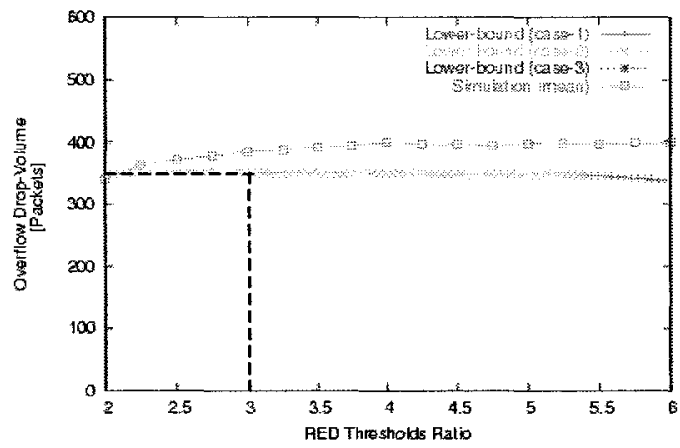

(d)

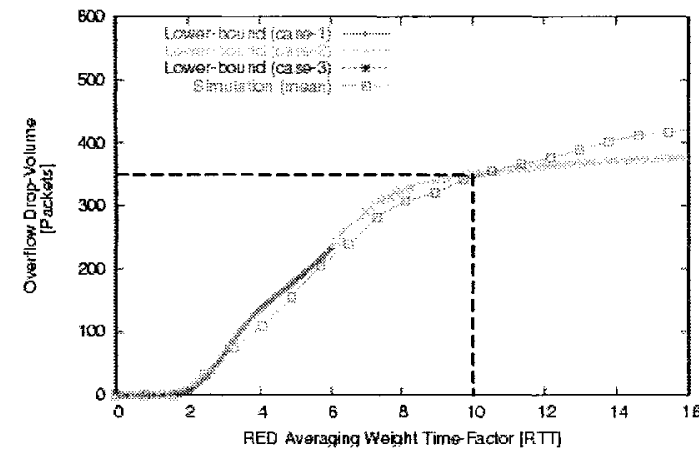

Fig. 4.2 Numerical Results for Overflow-based Drops Estimate $L_{V}^{\text {measure }}$ and Simulations Results vs. Varying: (a) Buffer-Size (b) Thresholds ratio (c) Target-Delay (d) Averaging Weight 


\subsection{Conclusions}

In this chapter we developed an analytical model for RED in CDMA-2000 data-networks with wireless links rate swings effects. A non-linear closed-form estimator on the volume of the data packet burst of losses due to buffer overflowing is derived from the model. The model allows us to reveal the deployed RED shared-queue's setting ranges for which performance-degrading effects are imminent, and distinguish those from preferred setting ranges.

Numerical solutions to the packet loss volume estimator are provided, and verified against simulations of equivalent systems. Low values for our estimator suggest preferred areas of operation for RED in which the calculated losses-volume estimator is small such that it possibly allows the TCP senders to avoid the costly RTO based recovery. These preferred tuning areas include: large queue buffer-sizes, low queue target-size values, and very large RED's averaging weight values. Furthermore, the numerical solutions show RED's inability to handle properly the transient congestion with random drops during the CDMA-2000 finite-burst mode when RED uses the literature tuning recommendations for wired-Internet.

In the next chapter we examine RED's performances as a function of its controls using a range of performance metrics. It demonstrates the range of effects of the different volumes of the closely clustered overflow-based drops. We then deduce final conclusions regarding the preferred tuning area for RED. 


\section{Simulation-Based RED Tune-Up for CDMA-2000}

A CDMA-2000 system constructed with a RED shared node and using the finite-burst mode of wireless links scheduling was shown in chapter 4 to be characterized with bandwidth variations causing large overflow-based bursts of data-packet drops.

In this chapter we extend the performance evaluation study of the sharp bandwidth increase effects via simulations at [4]. We test various TCP versions, present temporal system metrics, and allowing responsive (TCP) and unresponsive (UDP) traffic mix through the system. We show the boundaries of system performances, and identify distinct areas of operation as a function of the bottleneck queue's control parameters. We perform bottleneck RED queue tune-up.

The questions that this chapter targets are: What is the system response with different transport layers? What are the areas of operation for the bottleneck RED as a function of its controls? What are the corresponding system's performance boundaries? And lastly, what is the superior setting for the RED controls for high system performances?

We determine via simulation the best global area of operation in which there is a low sensitivity to the control parameters change. The superior solution at that area is suggested. Our tuned-up RED shows substantial improvement in the performance metrics compared to the wired-Internet tune-up recommendations at the literature. It shows an increase in throughput of up to $20 \%$ for backlogged FTP sources over TCP connections and tested system scenarios.

\subsection{Bandwidth Oscillations Extended Evaluation}

\subsubsection{Comparative Evaluation of TCP Versions}

In this section we consider various versions of TCP for performance analysis. We evaluate the most common deployed versions of TCP, namely: Tahoe, Reno, NewReno, 
Vegas, and the SACK extension. Aggregate normalized throughput is considered as the main performance metric (see section 3.4 for normalized throughput).

The Reno TCP achieves the lowest throughput, which is more than $25 \%$ lower than the other versions (see Fig. 5.1). The ACK compression based large burst of traffic results with large burst of data drops within a short interval at the PCF shared-queue. The Reno TCP connections suffer multiple packets losses at the same windows. Hence, the prolong timeout based recovery is required before restarting packets transmission [1].

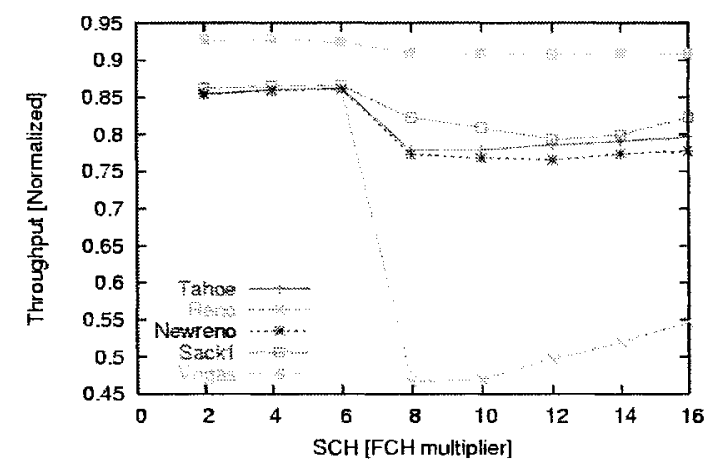

Fig. 5.1 TCP Versions Comparison

The Tahoe TCP-senders materialize the absence of the fast-recovery mechanism for re-initiate packets transmissions immediately after receiving 3 duplicated ACKs. By retuning to slow-start they "ignore" the large burst of drops, and despite the slower packets transmission rate they are able to maintain the flow of packets into the network after the SCHs allocations. The newReno, and SACK TCPs achieves high throughput comparing to Reno with our model. The newReno uses partial ACKs for clocking its Fast-Recovery and transmit new data, whereas SACK uses the explicit information in the ACKs to transmit the exact lost packets. The Vegas TCP implementation achieves the highest throughput in the face of the finite-burst mode rate-swing. Since it uses the RTT estimator to calculate the "precise" allowed transmission rate, its CWND does not depend on the incoming ACKs. Thus it avoids the ACK compression related adverse effects. 
As a conclusion, the commonly deployed Reno-TCP is the TCP version that is the most affected by the CDMA-2000's finite-burst, and therefore it is the focus of interest in this chapter in particular, and the thesis in general.

\subsubsection{System Throughput with Reno TCP}

The system's short-term normalized throughput is shown in Fig. 5.2. The simulation follows Fig. 3.1 structure with 100 long-lived Reno TCP connections each with FTP infinite greedy source. In-order to clearly present the AQM dynamics, burst interval of SCH allocation of 100 seconds to each MSs is used, and the inter bursts ('delay') interval is set to 50 seconds. The SCHs are concurrently allocated and de-allocated, which represents the worst-case scenario of wireless link scheduling. The PCF shared-queue is tuned according to $[6][10]$ recommendation.

A large period of close to 10 seconds of with virtually no new data delivery is observed closely after the SCHs allocations, proceeding with prolong throughput oscillations before the PCF queue is stabilized. It is a result of the ACK-compression based bandwidth-swings effects, as formalized at chapter 4 . These throughput effects become profound once shorter 'burst' intervals are assigned for the wireless links with the 'finite-burst' mode of scheduling.

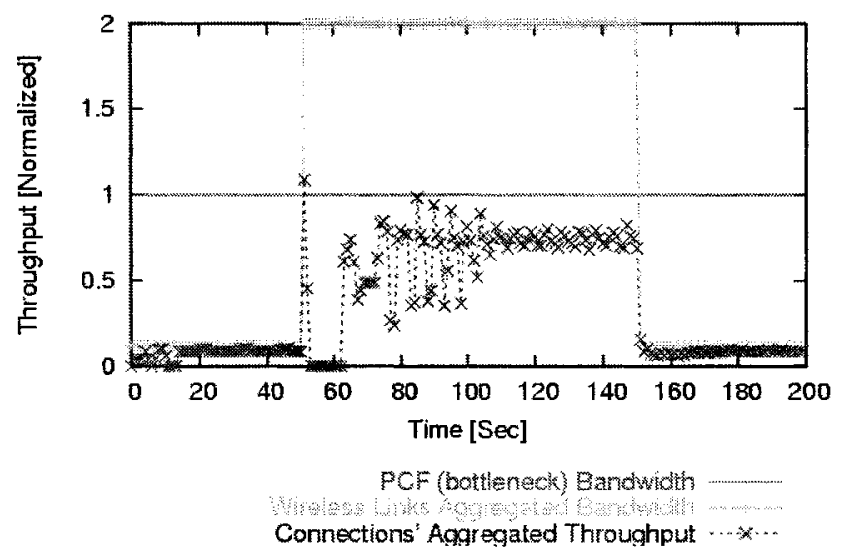

Fig. 5.2 System Throughput with ACK Compression in CDMA-2000 


\subsubsection{Unresponsive Traffic (UDP)}

The UDP lacks the TCP flow-control algorithms of ACK based and packets-loss based rate adjustments. It keeps transmitting at its full rate to meets its application rate requirement without adjusting the rate to the networks condition. At the CDMA-2000 context, the UDP traffic need to be policed at the PDSN using an upper bound of $\mathrm{FCH}+\mathrm{SCH}$ per connection in-order to avoid excessive UDP connections rate. Larger injected rate cannot be accommodated by the standardized wireless links bandwidth. It is further desired at a router to have a TCP-friendly UDP, at which the UDP application tunes its offered-load to match this of an equivalent TCP connection under the same system's congestion level [9]. As this may be the ultimate goal, currently large number of applications over UDP does not comply with this strategy. Thus, the PCF RED queue, which relies on packet-drops to covey the congestion indication for stabilizing the queue, can be largely affected by the UDP burstiness. On the other hand, for UDP connection an SCH allocation merely allows the link-queue to flush its buffered packets in a higher rate towards the sink, achieving higher throughput, with no ACK compression based degrading effects.

We explore the performance of CDMA-2000 finite burst mode with a mix of TCP Reno traffic and UDP traffic. The finite burst scheduling is set to delay interval of 10Sec, and burst interval of 5.2Sec. A CBR application over the UDP is used with uniformly distributed random inter-packets interval over $[-0.5 * 1 /$ rate, $+0.5 * 1 /$ rate $]$, with a mean rate of double the TCP-friendly rate.

The normalized throughput of the TCP traffic and UDP traffic is shown at Fig. 5.3. While the TCP connection's aggregated throughput exponentially decays with its percentage-wise number of connections, the UDP aggregated throughput grows exponentially. At small levels of aggregated UDP offered-loads, the UDP traffic materializes the TCP senders' back-offs closely after the SCHs allocations for occupying un-proportional space at the bottleneck-queue. Once the UDP aggregated offered-load 
become large enough compared to the TCP's, it practically inhibits the TCP senders' slow-start phase by aggressively occupying the shared-queue. Note that the total throughput slowly grows with the percentage of UDP connections since the UDP do not re-transmit packets upon their drops, which incurs lower data overheads.

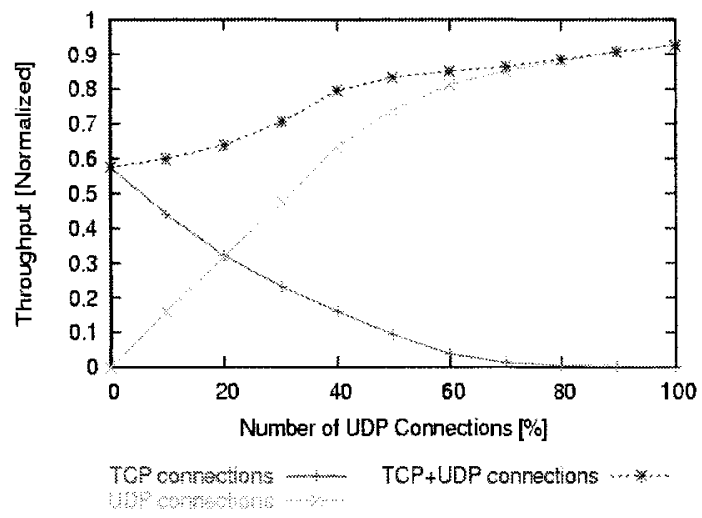

Fig. 5.3 Throughput vs. UDP-to-TCP Connections Ratio

\subsection{RED Tune-Up Evaluation}

In this chapter we perform RED bottleneck queuing tune-up via simulation in the context of CDMA-2000 SCH allocation related bandwidth swings. First, we perform extensive simulations to identify the PCF's RED queue performance boundaries. Then, we tune RED for the area of operation that achieves the best performance using a devised cost function.

The simulated CDMA-2000 reference model follows the system buffer scheme at Fig. 3.1 and the system setups at chapter 3 , with the following variations. The CDMA-2000 finite burst-mode repeatedly schedules 2.56 seconds of burst-time, and 10 seconds of delay-time. Three types of network traffic are analyzed. $80 \%$ of infinite backlogged longlived TCP connections are used with $20 \%$ of interactive TCP connections. We use interactive TCP connection to represent the more bursty traffic (see chapter 3.2 ). This allows us to examine the AQM characteristic of bias against bursty traffic. A mix of $80 \%$ long-lived TCP connections and 20\% UDP traffic is used as well. The UDP application is set with the EXP source (see chapter 3). The PCF's RED controls are listed at Table 3.1. 
Initial tests-set was executed to determine the performance metrics sensitivity to the RED controls, and their effective range. The queue averaging-factor $N$ (Eq. 2.7) was observed to have a profound impact on the RED queue behavior. We consider a range of values for $N$, starting from large number of RTTs for queue-averaging in which the queue was observed to fall-back to tail-drop behavior (i.e., the average queue size hardly ever reaches $t h_{\min }$, resulting with no early drops), and up-to zero number of RTTs for queue-averaging in which the RED queue practically acts upon its instantaneous occupancy. The ratio between $t h_{\max }$ and $t h_{\min }$ determines the desired range for the queue occupancy in which RED probabilistically drops packages. A too small gap can produces

oscillation at the queue where the average-queue goes from below $t h_{\min }$ (i.e., no drops) to above $t h_{\max }$. In particular, in Adaptive-RED a large enough thresholds ratio is required to avoid oscillations during $P_{\max }$ adaptation. The recommendation for the ratio is at least 2 [10] and typically 3 [8]. We vary this value from 2 to 4 . The buffer-size of the PCF queue, which represents the maximum packets capacity and ultimately bounds the maximum delay jitter at the queuing, is commonly expressed as a function (typically multiples) of the link capacity. In the simulation we present the buffer-size as a function of the target queue-size. This reflects its relation to the link-capacity while also account for the difference in the queue occupancy variations with varying target queue occupancy values. The RED's target average queue size sets the desired average delay at the queue during congestion. It represents a tradeoff for the system operator between throughput and delay, and is commonly set to be a fraction of the end-to-end round-trip time [6]. The following graphs focus on the results with queue target delay of 0.75 the RTT.

\subsubsection{System Boundaries and Areas of Operations}

The main performance metric of interest for the areas of operation is the data throughput curve in Fig. 5.4. It shows three main areas of operation of high performance with TCP 
traffic. These are described in the following. Note the viewpoint rotation around the $z-$ axis at the throughput performance graph.

The first area of high throughput is identified with large averaging factors $(>20 \mathrm{x}$ RTTs). However, in this area since the $q_{\text {ave }}$ only slowly adapts to $q_{\text {inst }}$ changes, the queue exhibits tail-drop dynamics after the SCHs allocations. The observed transient oscillations in the queue are large and persist throughout the burst time-interval. Thus, this area is susceptible to known tail-drop disadvantages of jitter as large as the queue's buffer-size (Fig. 5.4c) and large average-delay close to half the buffer-size regardless of the pre-configured target queue size (Fig. 5.1b). The various thresholds ratios in this area show un-significant change as the average queue-size only un-frequently exceeds $t h_{\min }$.

The second area of operation of high data through is identified with small queue averaging factors, ranging from 1 RTT down to 0 . This area was observed with the fastest response to the wireless-links aggregated rate increase and to the related ACKs compression based TCP senders' rate acceleration. The response to the rapid congestion build-up is at the form of rate throttling using probabilistic early drops sufficiently before the queue overflows, resulting with the smallest volume of overflow based deterministic drops. This in turn allows the TCP senders' to use their fast-recovery and gradually lower their transmission rates even at the face of continuing arrivals of compressed ACKs. The resulting average delay is observed to not exceed the pre-configured target, and low jitter at the queue is achieved. Thresholds ratio of at least 3 is sufficient for preventing overshooting $t h_{\max }$ or undershooting $t h_{\min }$ during most of the burst interval. The cost however of fast queue averaging is at the form of more restrictive allowable burst-size in the queue for those bursts that are desired to be accommodated at the queue (i.e., moderate bursts that does not overflow the queue).

The third and thin area of high throughput is identified with the smallest queue buffersize, close to twice the RED target fill $\left(=2 \cdot q_{t \arg e t}=2 \cdot(0.75 \cdot 2 \cdot D B P)=3 \cdot D B P\right)$ in between the two aforementioned queue-averaging factors areas, and ranging from 1RTT 
to 20RTT. This area is characterized with high data throughput with larger ratios for the RED thresholds. Low delay jitter is achieved subject to the small queue buffer size.

However, the largest number of queue overflowing intervals is experienced at this area.

The oscillations at the queue are as large as the queue size and persist throughout the burst interval. Hence the queue behaves as a tail-drop discipline, incurring the same disadvantages as described at the first area of operation.

The complementing area of operation to the above three, with substantially degraded throughput, is identified by queue averaging factor close to 10RTT, queue-size larger than twice RED upper threshold (i.e, about three times the target queue occupancy at the plots), and for all tested thresholds ratios. The queue's control parameters tune-up at this area closely represent the recommendations for the wired-Internet [6][10].
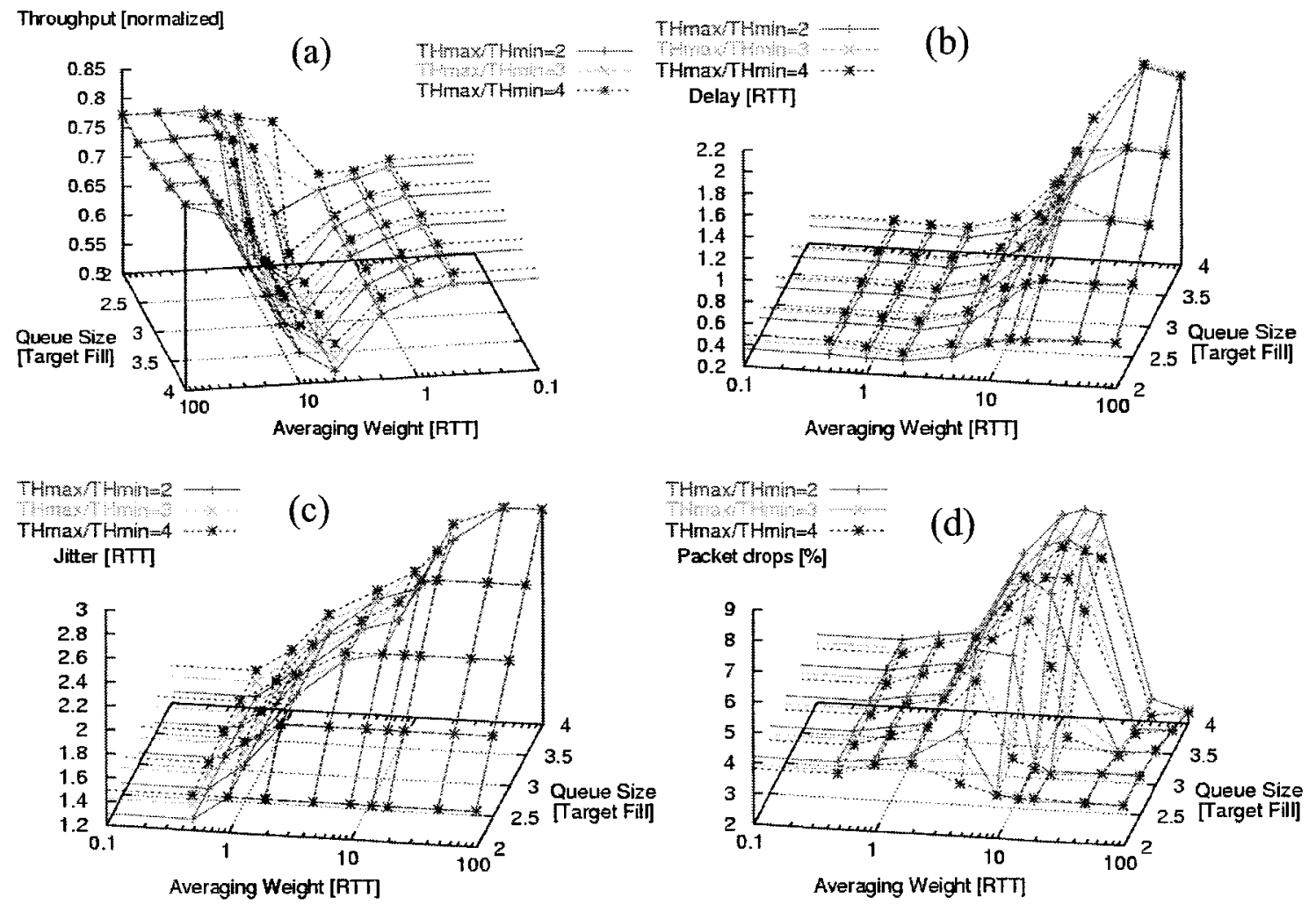


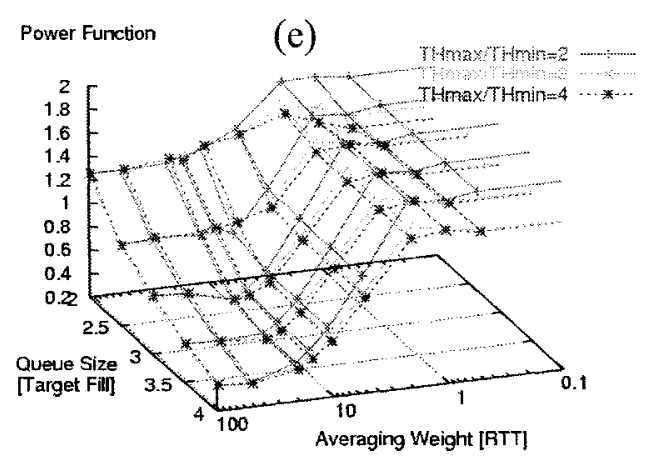

Fig. 5.4 TCP Performances vs. Bottleneck Queue's Controls (a) Throughput (b) Delay (c) Jitter (d) Data Packet Drops (e) Power Function

One goal of RED is avoid bias against bursty traffic [6]. In queue disciplines, such TailDrop, the more bursty the traffic from a particular connection, the more likely it is that the gateway queue will overflow when packets from that connection arrive at the gateway [34], hence penalizing the bursty connections with larger drop rates. In Fig. 5.5 we examine the aggregated throughput achieved by the group of interactive TCP connections, which represents the more bursty traffic flowing through the system. Fig. $5.5 \mathrm{~b}$ presents the throughput of the bursty group, whereas Fig. 5.5a shows the bursty group throughput as a percentage of total throughput. The areas of high throughput for the bursty group at Fig. 5.5b shows to match the three areas of high performance indicated above. We observe that RED tuned according to recommendation at [10] complement these areas and allow smaller throughput for the bursty group at the case of CDMA-2000 bandwidth swings.
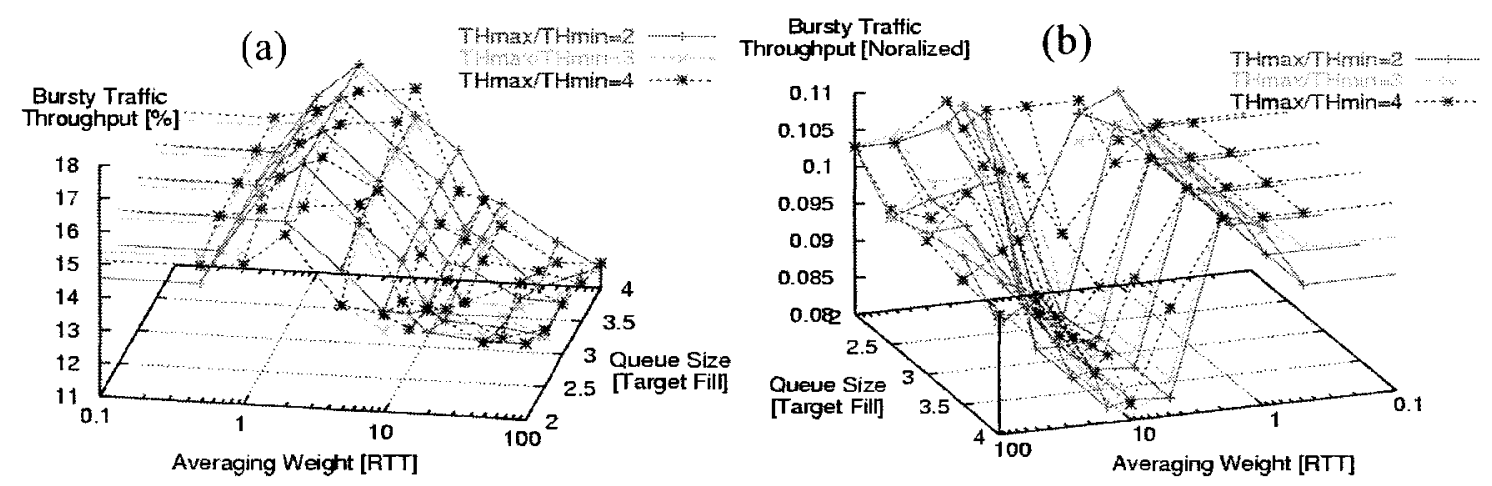

Fig. 5.5 Bias against Bursty TCP Traffic vs. Bottleneck Queue's Controls (a) Throughput percentage (b) Normalized throughput 
The data throughput curve in Fig. 5.6 shows the areas of operation of high performance with the aforementioned mix of TCP and UDP traffic. The areas of operations for highest performance follow those of the whole TCP traffic with the following variations. Higher throughput values are observed in all areas resulting from the UDP traffic sources' persistent sending rate and the UDP sinks acceptance of all arriving packets, both regardless of packet drops. Larger jitter and larger average delay values are observed. It results from the increased instability in the queue occupancy due to the UDP traffic sources burtiness and UDP unresponsiveness to congestion indications (i.e., packets drops).
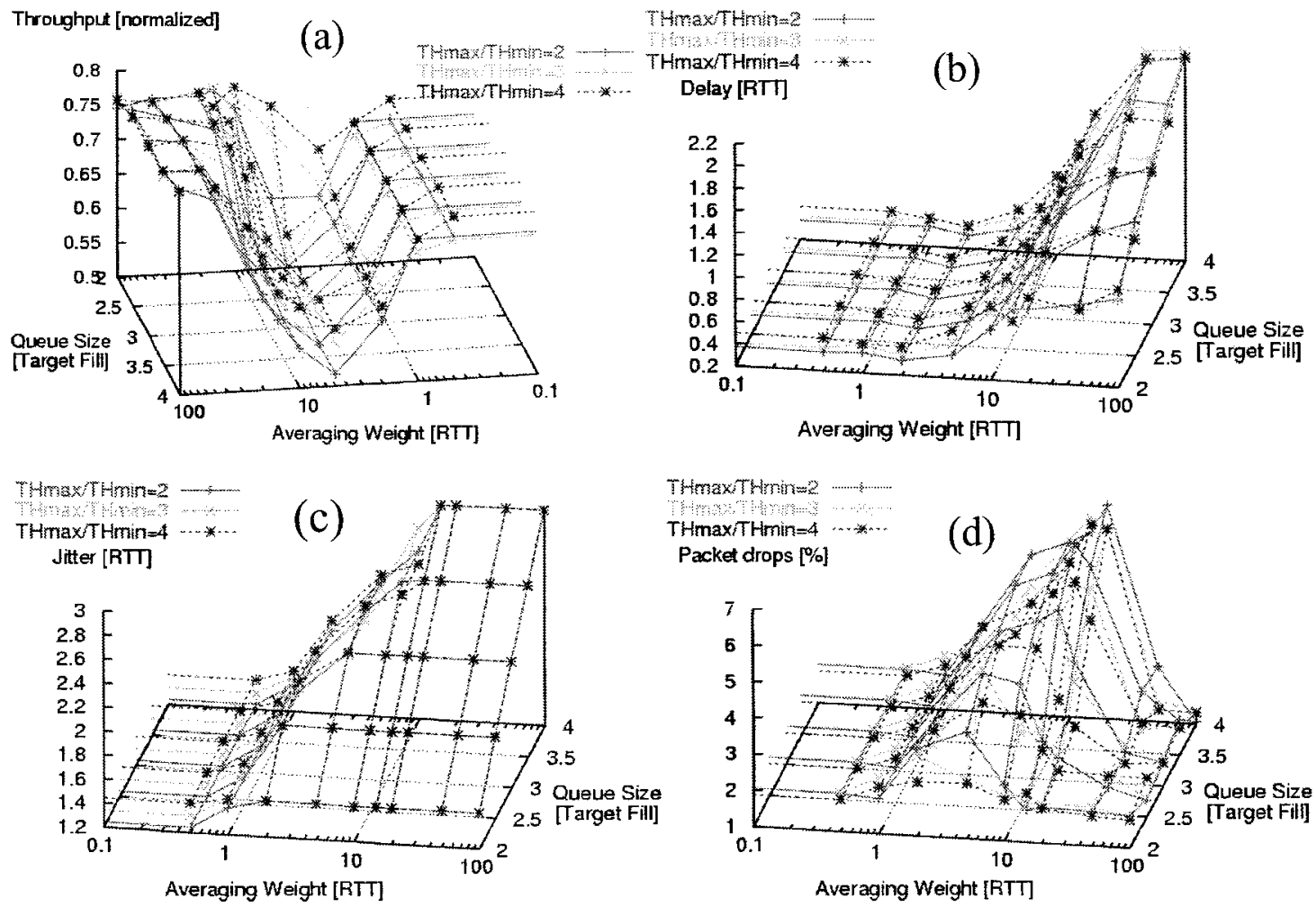


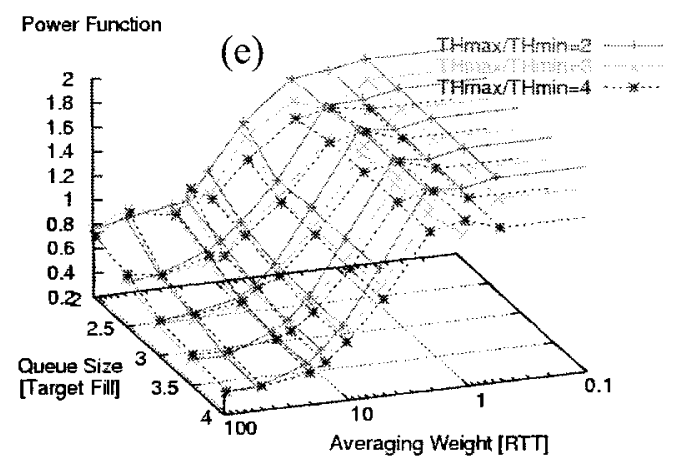

Fig. 5.6 Throughput of TCP and UDP Mix vs. Bottleneck Queue's Controls (a) Throughput (b) Delay (c) Jitter (d) Data Packet Drops (e) Power Function

\subsubsection{RED Tune-up}

This section presents a RED tune-up via simulation for CDMA-2000 data-networks with TCP connections, as well as with a mix of TCP and UDP connections. The objective is to identify the RED controls-set for best system performances. In-order to provide robust behavior, the tune-up first consider the global area of operation that maximizes performances with low sensitivity to controls changes, and then suggests the best RED controls-set at this area. Network simulation techniques are used to formulate the optimal configuration of RED as a black-box tune-up problem, considering the aforementioned parameters ranges.

For our CDMA-2000 model, there are numerous performance metrics to consider as inputs to the PCF's RED tune-up objective function. In particular, it is desired to maintain a high throughput while keeping the average queue size low and stable with using the lowest possible drops for throttling down the traffic. However, tuning-up for one performance metrics may compromise the other, and therefore an appropriate tradeoff has to be made. Essentially the multi-objective tune-up problem needs to be converted into a tractable single objective function. For converting multi-objective tuneup problem into a tractable single objective function, we propose an objective function that implicitly assigns weighted tradeoffs between the most important metrics: throughput, delay, and jitter. Tuning-up according to our objective function causes RED 
to settle in equilibrium status achieving high utilization, low queuing delays, and low jitter.

During the CDMA-2000 links' burst intervals, the average queue size of RED attempts to stabilize the congested queue around its target queue size. When the average queue-size overshoots the control of RED beyond $t h_{\max }$ the queue becomes unstable and oscillates between full and empty, incurring high jitter and underutilization periods. Thus, keeping the queue-size near its target-size is important for high throughput, as well as low delay and jitter. Such equilibrium also provides fewer overflow-based drops bursts, and thus greater fairness between flows. Hence, we define an objective function that quantifies the RED ability to retain the queue target size with the follows:

$$
O b j=\frac{\sum_{i=1}^{n}\left(q_{i n s t}^{i}-q_{t r g}\right)^{2}}{(n-1)}
$$

where $n$ is the number of samples of instantaneous queue fill $q_{\text {inst }}$ throughout the simulation. This essentially represents a loose term for stability at the queue at the form of the sampled variance for $q_{\text {inst }}$ with respect to the expected value $q_{t r g}$. For equilibrium level far from $q_{\text {trg }}$, which represents either excessive delays due to an under-dump condition with a too low drop rate, or an over-dump condition with excessive drop rate and lower throughput, the $O b j$ will be low. Likewise, if the equilibrium cannot be reached, the queue size will largely oscillate, again resulting in a low $O b j$. Thus, the $O b j$ function is a cost that needs to be minimized.

For the considered tuning problem of RED in this chapter, the desired tune-up algorithm is expected to converge to the global area of operation in which the sensitivity to changes in the controls is small. Moreover, high efficiency is required to allow large number of iterations with complex simulations. Taking the above features into account, and utilizing our system boundaries simulations, we choose a deterministic graphical tune-up technique, which is comprised of two stages: exploration, and exploitation. At 
the exploration stage the macroscopic features of the objective function is examined by iterating through large number of combinations of the parameters' value ranges, identifying promising areas of high stability in the parameter space. At the exploitation stage we exploit local information to improve the simulation resolution at the promising local areas, providing the best solution found.

The tune-up results for the objective function with TCP traffic shows a single area of operation with high stability. This area resides within the combination of averaging-factor values of 0.8 RTT down to 0 , and throughout all queue buffer-size values and thresholds ratios with insignificant influence of these two RED controls at that area. The small number of RTT required to average the queue suggest that a fast reaction to the wireless links' rate increase is needed in-order to stabilize the queue using mainly probabilistic drops. Indeed, the observed queuing dynamics in this area show that the PCF queue is stabilized using its RED mechanism, avoiding excessive queue overflow-based drops. It is also the reason for the wide range of queue buffer-sizes with maximized profit function. The tune-up results for the TCP traffic concluded a single area of operation with high stability. Fig. 5.7 shows the objective function's exploitation phase results constrained to the lowermost $10 \%$ values of the exploration phase $O b j$ results. Table 5.1 shows the tune-up results best setting for RED.

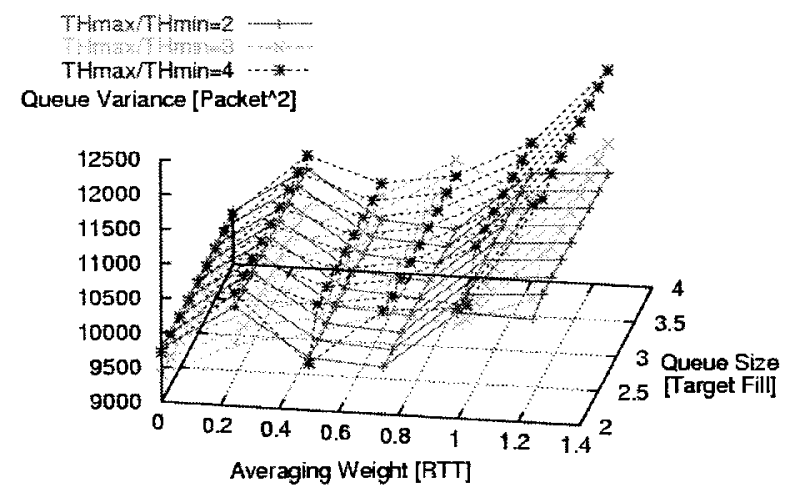

Fig. 5.7 RED Tune-up Objective Function with TCP (Exploitation Phase) 


\begin{tabular}{|l|l|l|l|l|l|l|}
\hline Control & $\mathbf{1}^{\text {st }}$ & $\mathbf{2}^{\text {nd }}$ & $\mathbf{3}^{\text {rd }}$ & $\mathbf{4}^{\text {th }}$ & $\mathbf{5}^{\text {th }}$ & Units \\
\hline Averaging factor & 0.5 & 0 & 0 & 0 & 0 & {$[$ RTT $]$} \\
\hline $\begin{array}{l}\text { buffer size } \\
\text { arget occupancy }\end{array}$ & 1.75 & 2.75 & 2.25 & 3.5 & 2 & \\
\hline$t h_{\max } / t_{\min }$ & 3 & 3 & 3 & 3 & 3 & \\
\hline Throughput & 0.674 & 0.677 & 0.677 & 0.677 & 0.677 & {$[$ Norm] } \\
\hline Average Delay & 45.613 & 43.667 & 43.671 & 43.665 & 43.660 & {$[\mathrm{msec}]$} \\
\hline Delay Jitter & 1.307 & 1.29 & 1.28 & 1.29 & 1.29 & {$[\mathrm{RTT}]$} \\
\hline Packet drop rate & 3.920 & 3.751 & 3.752 & 3.750 & 3.760 & {$[\%]$} \\
\hline
\end{tabular}

Table 5.1 RED Tune-Up Highest Results with TCP

The tune-up results for the mix of TCP and UDP traffic concluded a single area of operation with high stability. This area is also located at low averaging factor values of 1 RTT down to 0 , through all the queue buffer-size values, and mostly with larger values of thresholds ratios (Fig. 5.8). As in the whole TCP case above, the queue is stabilized better with small averaging factors for all tested buffer lengths. However, here the UDP sources sustain their transmission rate regardless of congestion notifications while the TCP senders back off closely after the SCHs allocations. Thus, higher throughput values are achieved. However the level of queue occupancy variations are subject to the UDP sources level of burstiness, where an increased UDP burstiness negatively affects the RED queue stability.

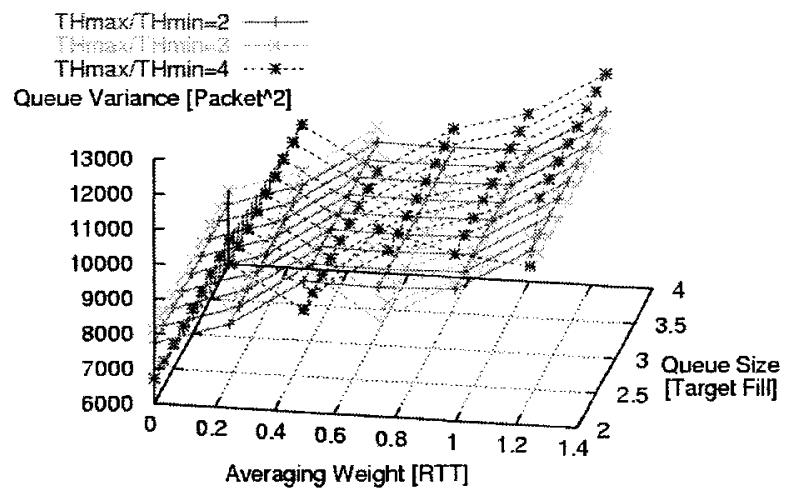

Fig. 5.8 RED Tune-up Objective Function with Traffic Mix of TCP and UDP (Exploitation Phase) 


\begin{tabular}{|l|l|l|l|l|l|l|}
\hline Control & $\mathbf{1}^{\text {st }}$ & $\mathbf{2}^{\text {nd }}$ & $\mathbf{3}^{\text {rd }}$ & $\mathbf{4}^{\text {th }}$ & $\mathbf{5}^{\text {th }}$ & Units \\
\hline Averaging factor & 0 & 0 & 0 & 0 & 0 & {$[$ RTT] } \\
\hline$\frac{\text { buffer size }}{\text { target occupancy }}$ & 1.75 & 4 & 3.5 & 2.75 & 3.25 & \\
\hline$t h_{\max } / t_{\min }$ & 4 & 4 & 4 & 4 & 4 & \\
\hline Throughput & 0.749 & 0.748 & 0.748 & 0.748 & 0.748 & {$[$ Norm] } \\
\hline Average Delay & 62.327 & 62.373 & 62.375 & 62.375 & 62.376 & [msec] \\
\hline Delay Jitter & 1.307 & 1.307 & 1.308 & 1.308 & 1.308 & {$[$ RTT] } \\
\hline
\end{tabular}

Table 5.2 RED Tune-Up Highest Results with Traffic Mix of TCP and UDP

\subsection{Conclusions}

In this chapter we targeted the evaluation via simulations of the RED mechanism as a function of its controls at the context of the CDMA-2000 bandwidth-swings.

We presented a comparative evaluation of the most commonly deployed versions of TCP. Reno TCP most under-performs causing large system under-utilization periods at the system. We presented simulation results of TCP and UDP concurrently flowing through the system. Adverse performance effects due to the interaction between the TCP connections and the non-responsive UDP connections in CDMA-2000 data-networks are shown.

Three areas of operation were identified with highest throughput as the main performance metric. The RED tuning recommendations for the wired-Internet reside in the complementing area with the lowest throughput and highest packer drop rate. The superior tuning area of RED was derived using an objective functions that combines various performance metrics of our interest. This tuning area is associated with very high queue-averaging weights. This area has negligible sensitivity to the buffer size and to RED's thresholds ratio values.

In the next chapter we introduce an alternative solution to mitigate the bandwidth swings effects at the CDMA-2000 networks. It was shown that RED's areas of operation of high 
performances also incurred pertaining costs. Therefore, the next chapter's solution attempts to maintain RED's tuning recommendation of Internet, while harnessing the buffer at the feeding node (i.e. the PDSN) to accommodate traffic during excessive congestion at the PCF. 


\section{Backpressure Feedback Flow-Control}

A common 'finite-burst' mode of wireless links scheduling in CDMA-2000 was shown to cause occupancy oscillations at the bottleneck shared-queue. The CDMA-2000 gateway (PDSN) node is constructed with superior service-rate compared to its downstream core node (PCF). The rate mismatch further allows for traffic load variations and subsequent congestion at the core node. The use of a Xoff/Xon feedback flow control in CDMA2000 was proposed at the $3 \mathrm{GPP} 2[17][18]$.

We evaluate the 3GPP2 backpressure proposal for protecting the bottleneck queue during excessive congestion conditions. We devise an Adaptive-Xoff/Xon for tandem queues, which extends the traditional Xoff/Xon [20][19] to provide threshold adaptation according to overflow prediction. The Adaptive-Xoff/Xon supplements the RED AQM at the bottleneck node, creating a hybrid flow-control model for tandem-nodes. It assists the RED mechanism during excessive congestion by eliminating the closely clustered overflow-based drops, while retaining the benefits of RED tuned with queue size averaging [10].

The questions that this chapter targets are: Can a feedback flow-control supplement a bottleneck CDMA-2000 router's AQM nodal flow-control for overflow protection and mitigation? What are the costs and benefits of such flow-control architecture? Furthermore, it questions whether a hybrid flow-control mechanism, which consists of backpressure flow control and an AQM at the bottleneck queue, is more effective in controlling network traffic than a nodal flow-control mechanism (i.e. the AQM) alone.

Experimental results show that the hybrid flow-control model eliminates overflowbased packet discards at the bottleneck queue, improves throughput, and lowers the data packets drop volume. Packets show to not experience backpressure-based delay variation while traversing the tandem queues. An associated cost is low volume of feedback control packets. Larger tandem-queues' average-delays are observed, which result with 
lower power-function. Hence, degraded combined performances are delivered by the 3GPP2 proposal for backpressure.

\subsection{Description of the Feedback Flow-Control Model}

Fig. 6.1 depicts a schematic view of the backpressure model. Data packets, flowing from the Internet hosts towards the mobile stations (MSs), traverse a system built of tandem queues at the CDMA-2000 system domain. The feedback flow-control intent to assist the RED mechanism in the PCF in confronting the phenomena of high volume of overflowbased burst of drops at the PCF after burst interval of the finite-burst scheduling mode at CDMA-2000. The backpressure thresholds are tuned in this chapter to be above the RED thresholds. The backpressure initiator communicates rate-throttling requests to the responder during such excessive transient congestions at the PCF queue. Excess traffic load is temporarily accommodated at the PDSN spare buffer space. Concurrently, the PCF RED mechanism generates rate-throttling requests to the traffic sources using packet-drops, targeting the steady-state convergence at the queue. Once sufficient convergence the PCF queue has been identified by the feedback flow-control mechanism such that no overflow events predicted, it retains a quiescence state. In quiescence state no control packets and no PDSN buffers due to backpressure-based rate throttling are required.

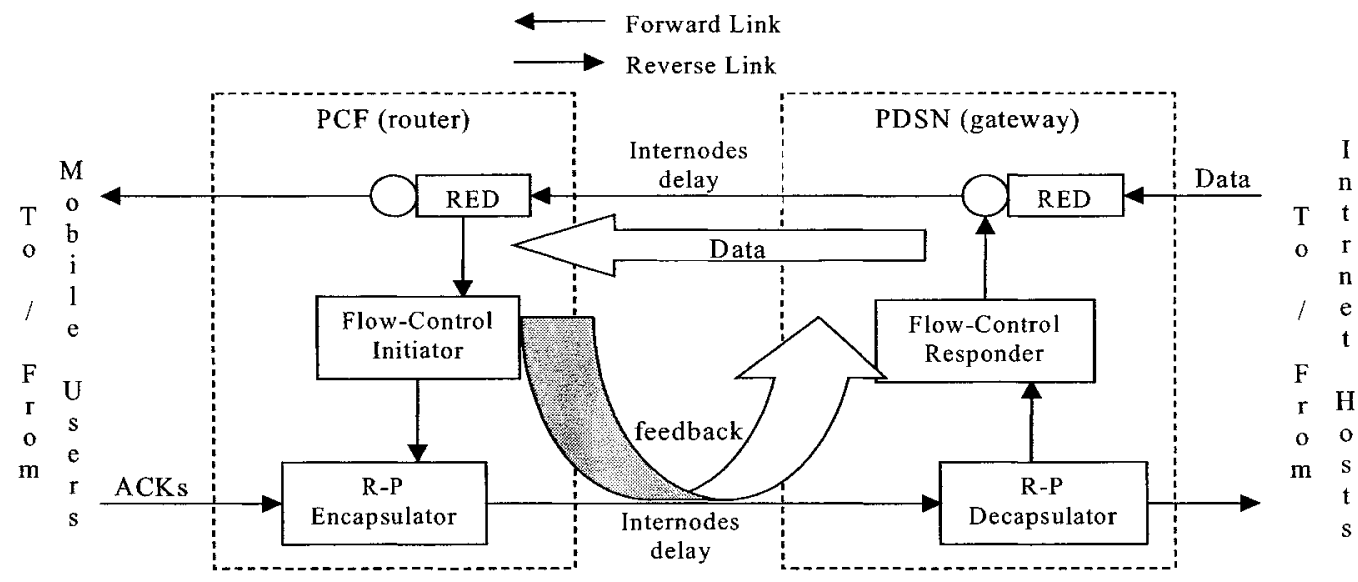

Fig. 6.1 Architectural View of the Tandem Queues Closed-Loop Feedback FlowControl for Single Sided Traffic 
The case of CDMA-2000 data-system introduces a specific set of topology, constraints, and assumptions for our backpressure mechanism. As mentioned, the PDSN gateway has superior service-rate. It is undesirable to load the PCF with additional processing, such as per flow state computation. Thus, in the context of the tandem queues we constraint the mechanism to react to aggregate of loads. Both involved nodes at the backpressure, the congested PCF and the feeding PDSN, are part of the same CDMA2000 cellular system. Thus, the parameters and characterization of the two nodes are mutually known, and these are programmed once at system setup time for the tandem queues feedback flow-control to later utilize. The stationary architectural topology presents fixed PCF to and from PDSN internodes propagation delay. Also, we assume negligible queuing delays for the backpressure's control-packets arriving at the PDSN. It is feasible due to a low volume of small sized control packets at our scheme, and the single sided-traffic that is largely characterizing today's $3 \mathrm{G}$ cellular networks.

Three components can be identified at the proposed Adaptive-Xoff/Xon flow-control mechanism: detection, notification and action. These are discussed below.

\subsubsection{Congestion Detection}

The PCF congested node implements a monitoring mechanism, which detects and signals the occurrence as well as the end of excessive congestion. This mechanism is based on the buffer occupancy at the PCF shared-queue, and a dual thresholds adaptive scheme.

The feedback loop delay interval is a function of the internodes propagation delay, and the control packets transmission time interval:

$$
D_{\text {loop }}=2 \cdot D_{\text {prop }}+\frac{L_{c n t r}}{r_{P C F}}
$$

where $D_{\text {prop }}$ is the PDSN to/from PCF propagation delay, $L_{c n t r}$ is the size of a dedicated control packet, and $r_{P C F}$ is the service rate of the PCF. We assumed identical PCF service-rates at the forward and reverse directions. 
Once either an Xon or an Xoff indication was dispatched from the PCF toward the PDSN, the PCF queue occupancy trend may persist before the control action affects the traffic at the PCF's ingress. The potential PCF's queue occupancy increase after the backpressure upper threshold was exceeded and an Xoff feedback indication was sent is:

$$
\Delta Q_{P C F}^{\text {Xoff }}=D_{\text {loop }} \cdot R_{\text {mismatch }}^{\text {Xon }}
$$

where $R_{\text {mismatch }}^{\text {Xon }}$ is the rate mismatch between the PCF's inflow to outflow during Xon interval at the PDSN. Equivalently, the potential PCF's queue occupancy decrease after the backpressure lower threshold was crossed down and an Xon feedback indication was sent is:

$$
\Delta Q_{P C F}^{\text {Xon }}=D_{\text {loop }} \cdot R_{\text {mismatch }}^{\text {Xoff }}
$$

where $R_{\text {mismatch }}^{\text {Xoff }}$ is the rate mismatch between the PCF outflow to inflow during Xoff interval at the PDSN.

The backpressure's thresholds should at the least be tune to attempt and protect the PCF queue from overflow and underflow scenarios during congestion. Consecutively, the following necessary condition needs to be satisfied:

$$
\Delta Q_{P C F}^{X o f f}+\Delta Q_{P C F}^{X o n} \leq B_{P C F}
$$

where $B_{P C F}$ is the PCF buffer size. Considering variable levels of traffic loads, with the using fixed thresholds for the backpressure $R_{\text {mismatch }}^{\text {Xon }}$ needs to represent the worst-case scenario, which is the service-rate mismatch between the PCF and the (un-throttled) PDSN: $R_{\text {mismatch }}^{\text {Xon }}=R_{P D S N}-R_{P C F}$. Under the assumption of sufficient load at the PDSN and neglecting probabilistic early drops at the PCF queues, $R_{\text {mismatch }}^{\text {Xon }}$ represents the worst-case PCF queue fill growth rate after an Xoff was sent. However, with common PDSN to PCF service rate mismatches and bounded PCF buffer sizes, the resulting backpressure upper threshold requires very low values. The potential increase in the PCF's buffer occupancy between the time of an Xoff generation and the rate reduction at the PCF ingress can be 
as large as the total PCF's buffer size, or even greater. Hence, it is impractical to set both thresholds to satisfy Eq.(6.4). Note that $\Delta Q_{P C F}^{X o n}$, on the other hand is independent of the PDSN full rate, and for practical PCF rates and buffer sizes it is substantially smaller than $\Delta Q_{P C F}^{\text {Xoff }}$

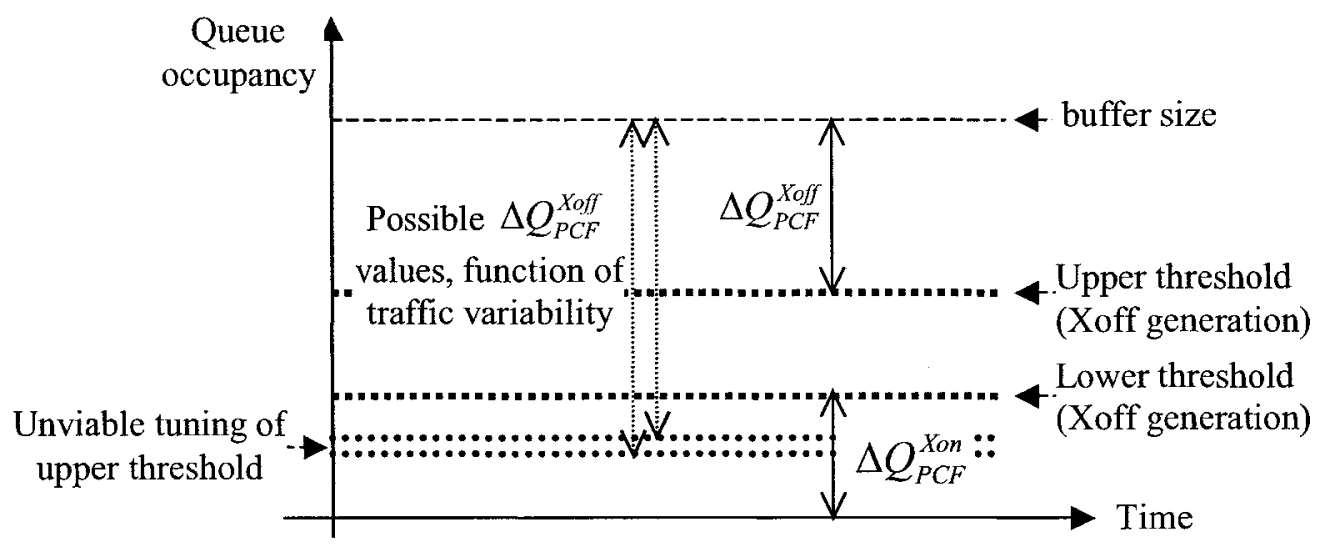

Fig. 6.2 Difficulty in Tuning Xoff/Xoff Backpressure's Fixed Thresholds

Our Adaptive-Xoff/Xon includes a mechanism at the PCF to predict queue overflow events and send feedback notifications in advance. The mechanism addresses the impractical tuning of fixed thresholds at the traditional Xoff/Xon while allowing for minimizing the overflow events at the PCF even during excessive congestion (e.g., CDMA-2000 bandwidth-swings). Consecutively, the upper threshold is not fixed, but rather a variable and continuously adapted threshold with (discrete) time. An estimator on the smoothed queue growth-rate is calculated using a low-pass filter on the queue's instantaneous growth-rate. The calculations take place at any packets departure from the PCF. It can be written as:

$$
r_{Q}[i+1]=w \cdot r_{Q}[i]+(1-w) \cdot r[i+1]
$$

where $r_{Q}$ is the smoothed growth-rate, $w$ is a smoothing weight, and $r$ is the instantaneous growth-rate between two subsequent packets departures. An overflow event is predicted (for an assumed sustained arrivals rate till overflow) if the following inequality is satisfied: 


$$
r_{Q}[i] \cdot D_{\text {loop }}+q_{S}^{\text {inst }}[i]>t h_{t \text { arget }}^{\text {Xoff }}
$$

where $q_{S}^{\text {inst }}$ is the PCF buffer's instantaneous occupancy, and $t h_{t \text { arget }}^{\text {Xoff }}$ is the desired maximum buffer fill. Therefore, the adaptive variable (with discrete time) upper threshold $t h_{\text {gen }}^{X o f}[i]$ can now be written as:

$$
t h_{\text {gen }}^{\text {Xoff }}[i]=\min \left(t h_{t \text { arget }}^{\text {Xoff }}-r_{Q}[i] \cdot D_{\text {loop }}, \quad t h_{t \text { arget }}^{\text {Xoff }}\right)
$$

The lower target queue fill can equivalently be:

$$
t h_{\text {gen }}^{\text {Xon }}=t h_{t \text { arget }}^{\text {Xon }}+R_{\text {mismatch }}^{X o f f} \cdot D_{\text {loop }}
$$

Consecutively, the system operator needs only to provide the target levels $t h_{t \text { arget }}^{\text {Xoff }}$ and $t h_{t \text { arg } e t}^{X o n}$ for the backpressure mechanism to attempt to be maintained. In the simulations, we express the target upper and lower thresholds through the terms: hysteresis-center and hysteresis-size, denoted as $C$, and $H$, respectively. The relationships are:

$$
\begin{aligned}
& t h_{t \text { arget }}^{\text {Xon }}=C-\frac{H}{2} \\
& t h_{\text {target }}^{\text {Xoff }}=C+\frac{H}{2}
\end{aligned}
$$

The decision scheme for sending Xoff and Xon indications is visualized at Fig. 6.3. The lower threshold is calculated a priori using Eq.(6.8), whereas the upper threshold is adaptively calculated using Eq.(6.7).

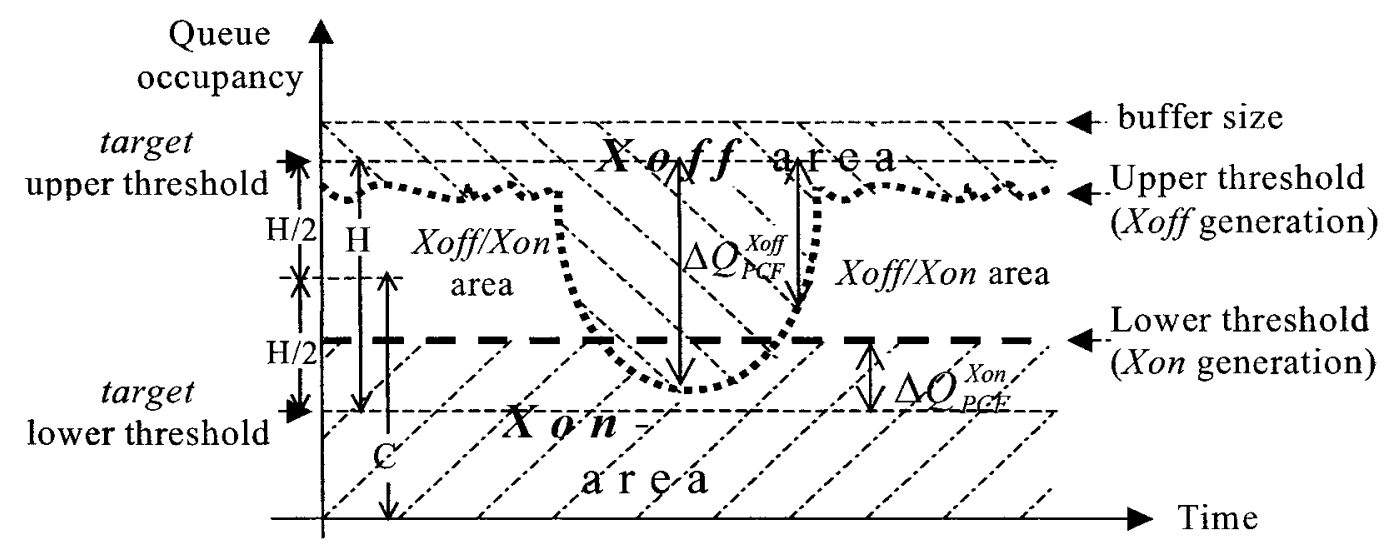


Fig. 6.3 Upper and Lower Thresholds Interaction in Adaptive-Xoff/Xon Scheme

The Xoff area includes any buffer fill values for which the queue fill is larger or equal the upper threshold, whereas the Xon area is restricted to be smaller than both the lower the upper threshold. The reminder area (blank) is considered an Xon or Xoff area if the most recent control-packet carried an Xon or Xoff indication, respectively. Note that subject to the upper threshold's adaptive change, a scenario in which the upper threshold falls below the lower threshold may occur.

Let's define Xoff (Xon) state at the PCF if the most recent control-packet carried an Xoff (Xon) indication. Then, the decision process for the feedback indication follows a simple routine:

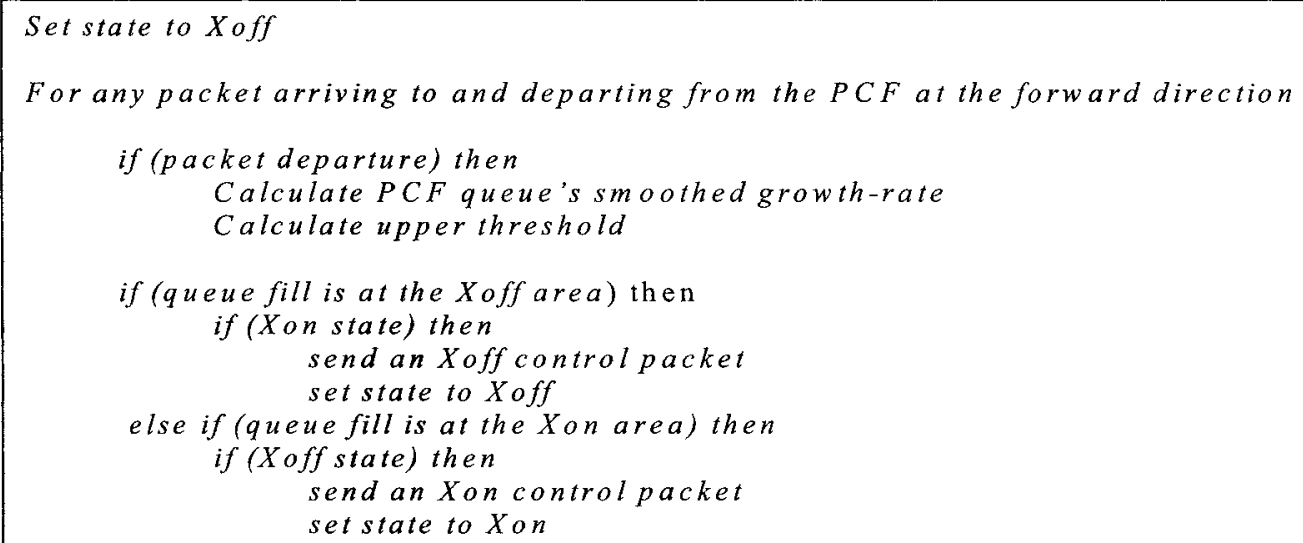

Fig. 6.4 Adaptive-Xoff/Xon Control Packet Submission Decision Routine

\subsubsection{Notification}

This section depicts the choices of notification delivery methods made by the AdaptiveXoff/Xon mechanism.

The Adaptive-Xoff/Xon backpressure uses the A10 bearer path between the PCF and the PDSN to covey the feedback control packets. The A10 data bearer channel is assigned with higher processing priority (compared to A11 signaling channel), hence minimizes the control loop latency. The Adaptive-Xoff/Xon distributes the feedback telemetry overhead between the active connections by transmitting a control packet to the PDSN on the A10 virtual channel of the most recent arriving packet to the PCF. 
The single bit control indication may be carried by GRE encapsulation either within a dedicated control packet, or an ACK (or data packet for dual-sided traffic), flowing at the reverse direction. Our study of the backpressure concentrates on delivering the feedback indication using dedicated packets.

\subsubsection{Control Actions}

When the feeding PDSN node is notified of congestion occurring (or ending) at the PCF it has to perform control actions that would alleviate the congestion (or proceed to reverse the control actions taken previously).

In our study, we consider a scheme where the control actions and reverse actions are transmission throttling-up/down, respectively. The control role $u$ at the PDSN is:

$$
u= \begin{cases}\text { Throttle down to Xoff rate, } & \text { if bit is set in GRE feedback attribute } \\ \text { Throttle up to Xon rate, } & \text { if bit is not set in GRE feedback attribute } \\ \text { Maintain rate, } & \text { if GRE feedback attribute is not encapsulated }\end{cases}
$$

A 'Xon rate' allows the PDSN to transmitted up to its full service-rate. An 'Xoff rate' instructs the PDSN to throttle down its transmission rate to a pre-programmed value. The PDSN uses the knowledge of the PCF rate and determine the throttle down value to be a fraction of the PCF service rate. Any such fraction value eventually causes the PCF buffer occupancy to decline..

The Adaptive-Xoff/Xon scheme utilize a timer at the PDSN for timing-out Xoff related control actions in the rare event of an Xon control packet loss or corruption. The PDSN implicitly determine the Xoff elapse time once at setup time to equal the longest time interval that takes the PCF queue to empty a full queue (i.e., assuming target upper threshold level equals the PCF buffer size and sustainable arriving traffic load from the PDSN after an Xoff indication reception):

$$
\Delta t_{P D S N}^{\text {elapse }}=\frac{B_{P C F}}{R_{\text {mismatch }}^{\text {Xoff }}}
$$


These quantities are of fixed values, and are to be input to the PDSN once during the system setup time.

\subsection{Simulation Results and Discussion}

In this section we evaluate the hybrid flow-control using simulations. $\mathrm{C}++$ models of our hybrid flow-control are used and exercised under the popular NS-2 [33] simulator.

\subsubsection{Simulations Setup}

This section describes the default traffic and system setups in addition to the described at chapter 3 . These setups are used in the proceeding system performances evaluation scenarios. In the various tests we may vary one or more of the setup's parameters.

The system carries 100 active flows, each using an infinite backlogged FTP source over TCP connection. Referring to CDMA-2000 reference model (Fig. 3.1), the PDSN may be congested only upon backpressure's rate throttling. The PDSN's service-rate can be approximated with the PCF service-rate during excessive PCF congestion before the PCF queue is stabilized below the backpressure thresholds to its target occupancy by the RED mechanism. During this interval, the backpressure mechanism stabilizes the PCF queue in-between the backpressure's thresholds for avoiding overflow events by matching the PDSN outflow rate to the PCF out-link rate. Hence, to avoid excessive delays and jitters at the PDSN queue during its congestion interval, the PDSN RED and buffering characteristics are set as a function of the approximated service-rate, and therefore equals those of the PCF's.

The parameters and characterization of the two involved nodes at the tandem-queues backpressure are mutually known. These two nodes are considered to be under the same cellular service provider, which enable to program the required parameters for the backpressure at setup time. In particular, the PCF is being preprogrammed with the PCF to PDSN internodes propagation delay, as well as the Xoff service-rate at the PDSN. The PDSN is preprogrammed with the PCF service-rate, the Xoff service-rate value, and the 
PCF buffer size. These parameters are then utilized to determine the fixed metrics once at setup time, as well as the adaptive metric. The fixed metrics includes the lower threshold level at the PCF, and the implicit timeout timer initial value at the PDSN. The adaptive metrics includes the discrete time upper threshold level at the PCF. In accordance, these parameters are presented for the PCF and the PDSN at each test, and are modified for each simulation's iteration as noted at the proceeding text or graphs.

An Xoff indication rate instructs the PDSN to throttle down its transmission rate. Slower occupancy declining at the PCF means smaller number and less sharp buffer's occupancy oscillation at the PCF till the queue is stabilized by the RED mechanism. Therefore, we choose a rate fraction of 0.9 the PCF rate.

We ran experiments with the weight of the smoothed queue-fill growth-rate $(w)$ in the value range of $[0.1,0.01]$. Negligible performance differences were observed for the backpressure' thresholds overshooting and overflow-based packet drop volume. Therefore, we set $w$ at the following simulations to a fix value of 0.1 .

The backpressure thresholds are set to be above the target congestion at the PCF queue. This way, the backpressure operation results with no congestion and delays at the PDSN queue and no control packets overhead once the PCF queue has been sufficiently stabilized by RED to not exceed the backpressure upper threshold. The backpressure's upper threshold is set close to the PCF buffer size while allowing for small occupancy margin from a full buffer size. This way, the PCF is protected from overflowing while maintaining large enough hysteresis to reduce the volume of control-packets. Accordingly, the hysteresis-center $(C)$ and hysteresis-size $(H)$ are set for the backpressure to operate above the RED upper threshold, with values of $70 \%$ and $40 \%$ of the PCF buffer size, respectively. 


\subsubsection{Temporal Queues and System Dynamics}

This section presents the backpressure model's temporal dynamics. The simulation system in this test scenario is constructed with 140 TCP connections and a burst interval of 30 seconds to clearly demonstrate the queuing dynamics.

The simulation results are shown in Fig. 6.5. Fig. 6.5a,b show the occupancy levels at the PCF and PDSN queues. The backpressure metrics are labeled with 'BP'. Note the coinciding of the backpressure's lower threshold with RED's upper threshold. The backpressure protects the PCF queue from overflowing in each of the observed finiteburst mode related bandwidth swings. Concurrently, RED dumps the queuing occupancy oscillations through early drops (also Fig. 6.5d). The backpressure predicts an overshooting of its upper threshold by using the calculation of the PCF queue smoothed growth-rate (Fig. 6.5c), and adapting the upper threshold accordingly. The smoothed rate calculation shows both positive and negative values, which corresponds to occupancy increase and decline, respectively. The positive rates and zeroed negative rates are considered for adapting the upper threshold. The backpressure-based queue occupancy variations shown to be bounded between the backpressure upper and lower thresholds, above the RED upper threshold.

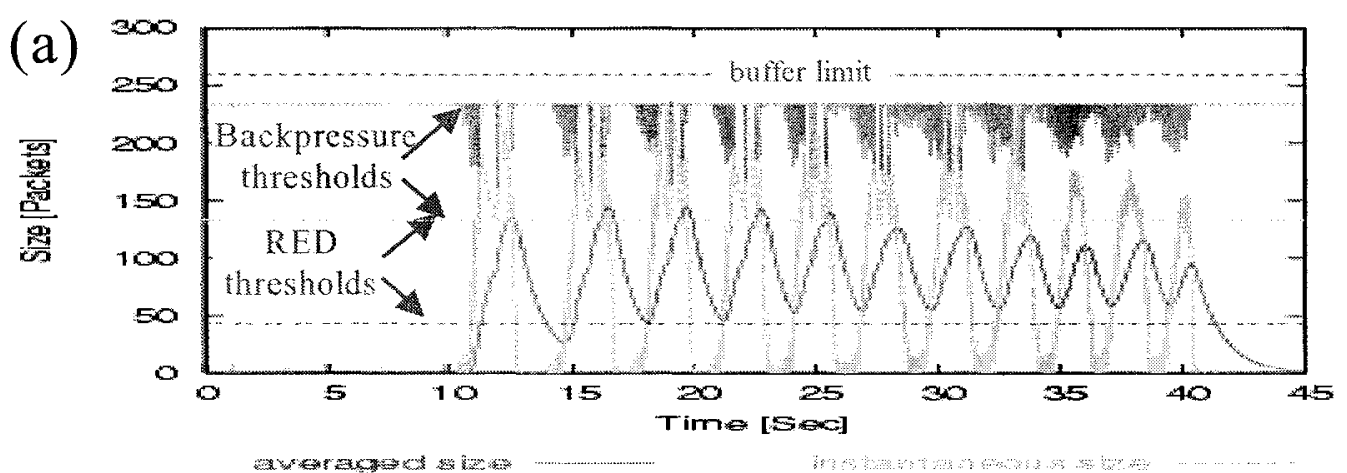




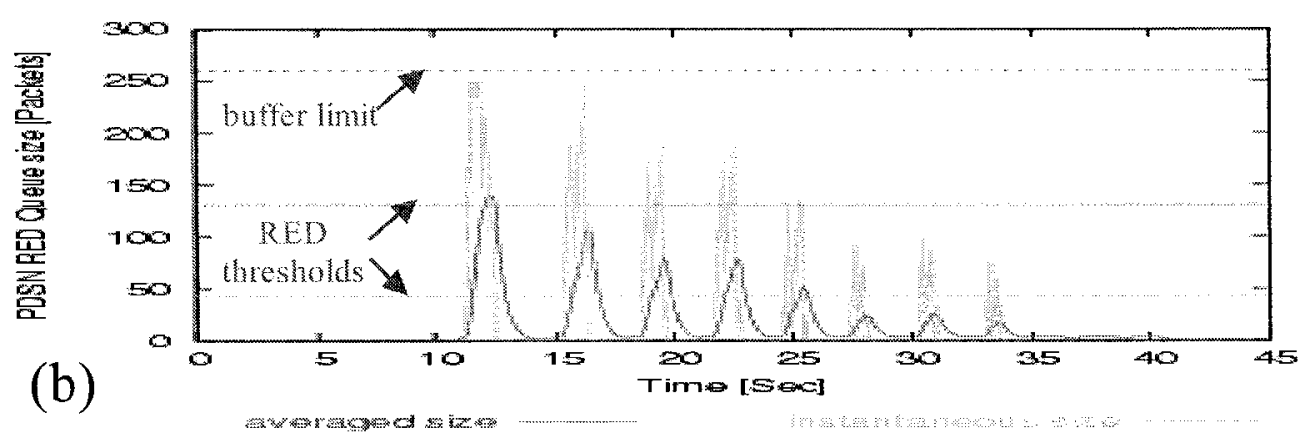

(c)

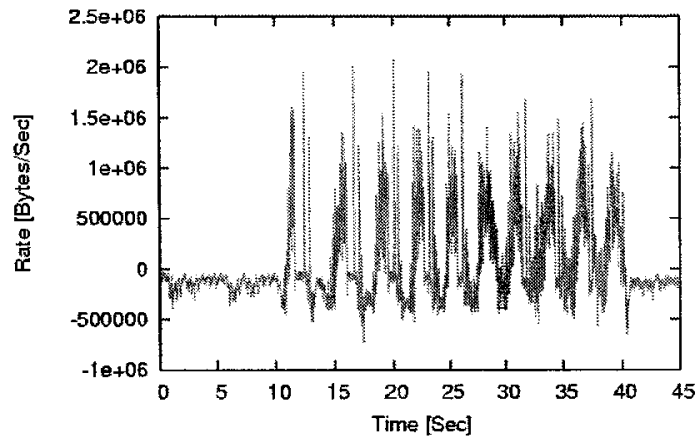

(d)

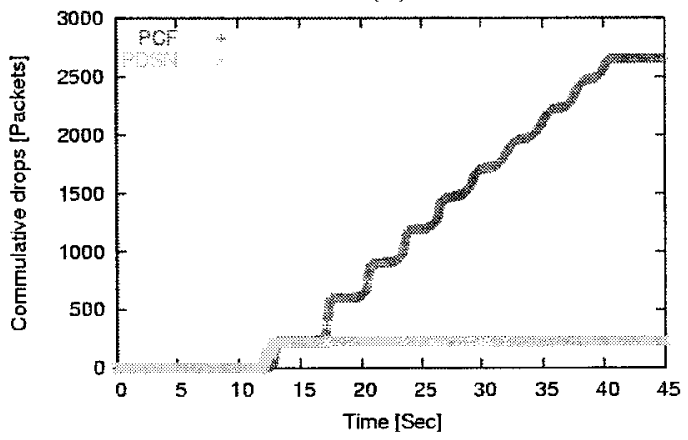

Fig. 6.5 Temporal queuing and backpressure dynamics (a) PCF thresholds and occupancy levels (b) PDSN thresholds and occupancy levels (c) calculated occupancy smoothed rate (d) cumulative data packet drops

The temporal queuing delays in the tandem queues are shown at Fig. 6.6. The results demonstrate that for CDMA-2000 case of tandem queues, the occupancy oscillations do not translate into delay oscillations. That is, the PCF and PDSN queuing delays sum to a delay envelope, which is governed rather by the bandwidth swings effects. Backpressure based delay variations were observed only during harsh bursts such that the PDSN is overflowed. Note the moderate occupancy slope during an Xoff state compared to the slope during an Xon state. This results from the Xoff rate factor of 0.9 , and the rate mismatch factor of 3 . 


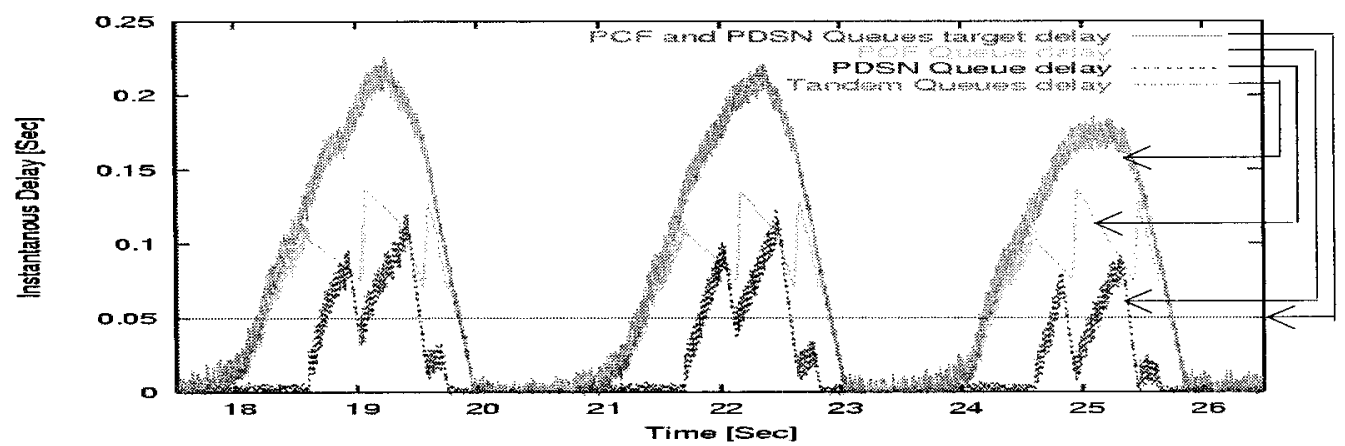

Fig. 6.6 Temporal queuing delays

\subsubsection{The Effect of Congestion Depth}

We evaluate the hybrid flow-control model with number of connections ranging from 30 to 150 active connections. This represents varied PCF overload levels and consequent congestion depths (defined as the ratio between the PCF ingress and egress rates). We compare the hybrid model with a nodal flow-control. The nodal model is constructed with a PCF RED queue without backpressure.

Fig. 6.7 demonstrates several performance benefits of the hybrid flow-control. Improved aggregated throughput is observed once the queues are congested. Less data packets are dropped in the tandem queues, while no overflow-based drops are experienced at the PCF with all traffic load levels.

Fig. 6.7 demonstrates several performance benefits of the hybrid flow-control. We observe the congestion built-up with less than 40 users, the congestion knee point at 40 users, and the congestion collapse with more than 40 users. Improved aggregated throughput is observed once the queues are congested. Less data packets are dropped in the tandem queues, while no overflow-based drops are experienced at the PCF with all traffic load levels. 
(a)

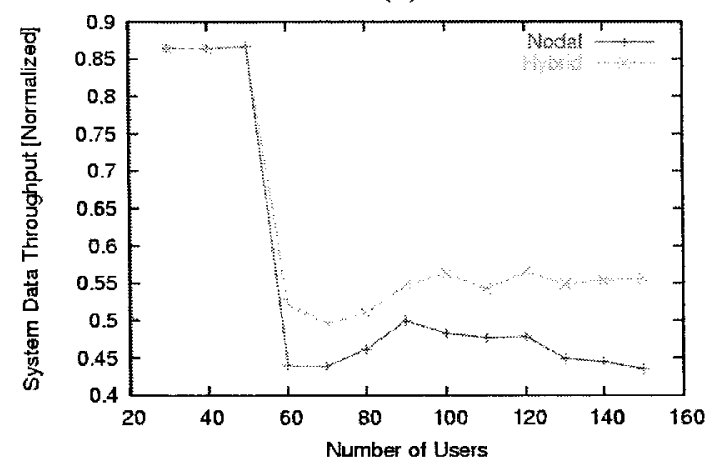

(b)

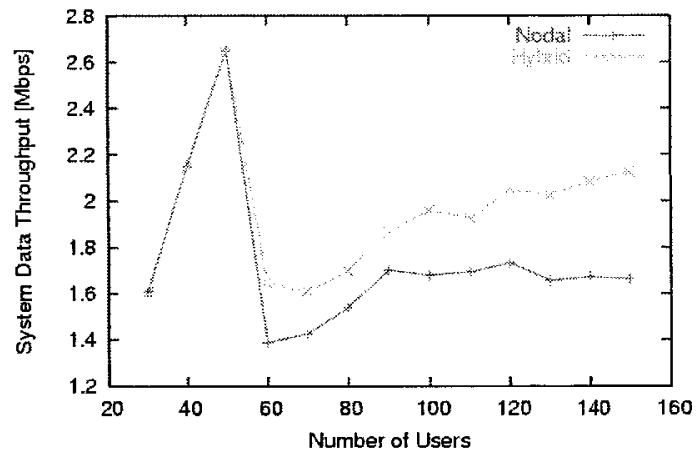

(c)

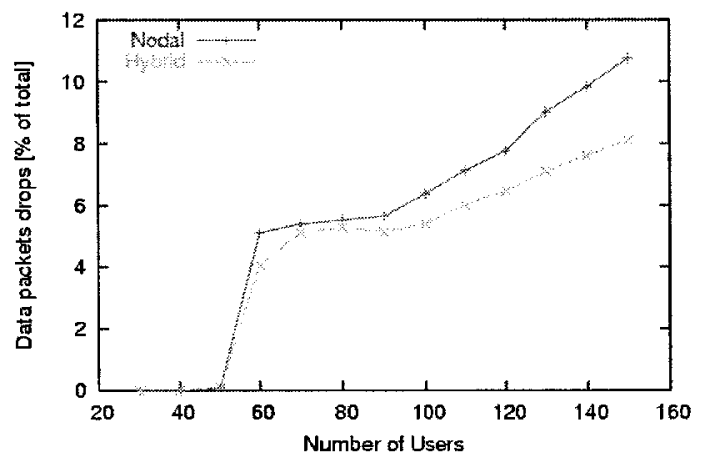

Fig. 6.7 Backpressure model's performances benefits as a function of the congestiondepth (a)(b) throughput (c) tandem-queues' data packet drop rate

Fig. 6.8 shows the performance costs associated with the hybrid flow-control. Low percentage of control packets is observed, compared to the volume of data packet that it supports. The backpressure uses PDSN free buffer space to accommodate excessive traffic loads. It results with larger average delays measured during 'burst' intervals at the tandem queues.

(a)

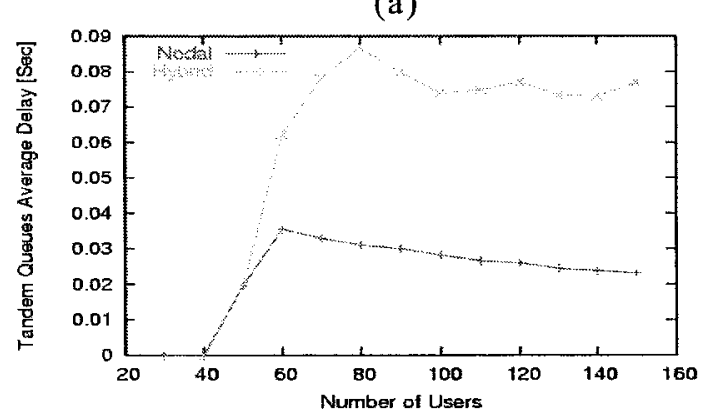

(b)

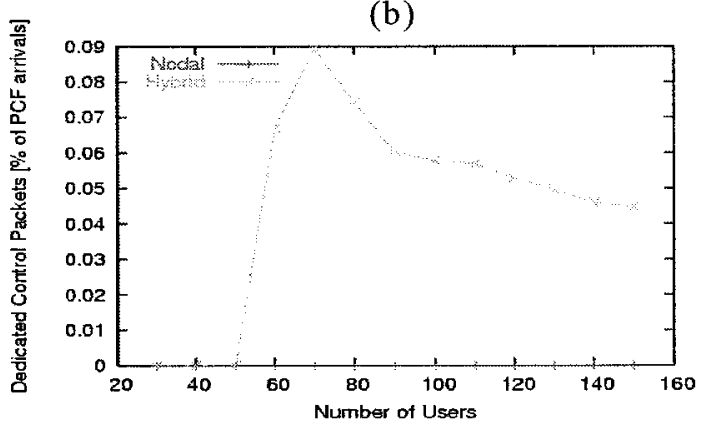

Fig. 6.8 Backpressure model's performances costs as a function of the congestion depth (a) tandem-queues average-delay during 'burst' intervals (b) overhead of control packets 


\subsubsection{The Effect of Congestion Duration}

We evaluate the hybrid flow-control model with various congestion durations, using a range of SCH allocation intervals. We use a set of values to represent both 'finite-burst' and 'infinite-burst' wireless link scheduling modes (see chapter 2.1). The values set used for the burst time interval is: $0.16,0.32,0.64,1.28,2.56,5.12,10.24,20.48,40.96,81.92$, and 163.84 [seconds]. The first 6 values $(0.16$ - 5.12) are taken from the CDMA-2000 standard for 'finite-burst', and the rest are arbitrarily chosen in the 'infinite-burst' range. We compare the hybrid model with a nodal flow-control. The nodal model is constructed with a PCF RED queue without backpressure.

Fig. 6.9 demonstrates several performance benefits of the hybrid flow-control. Improved aggregated throughput is observed for all burst intervals. Less data packets are dropped in the tandem queues, while no overflow-based drop events are experienced at the PCF with all the 'burst' intervals.

(a)

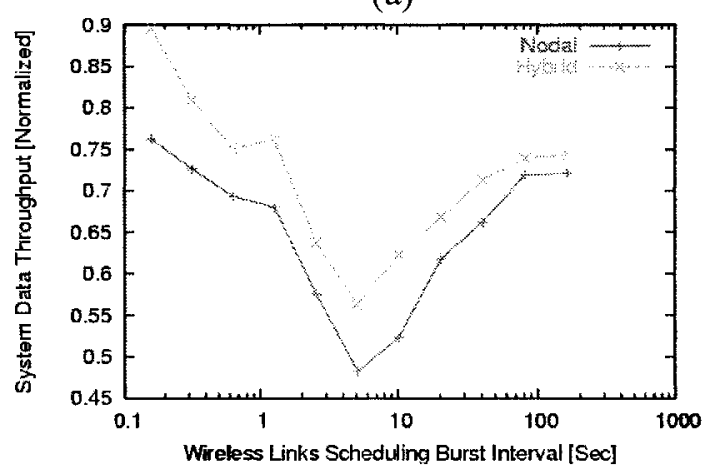

(b)

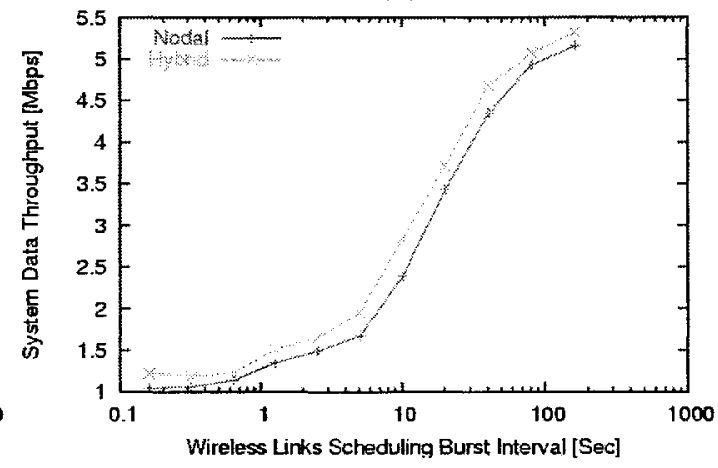

(c)

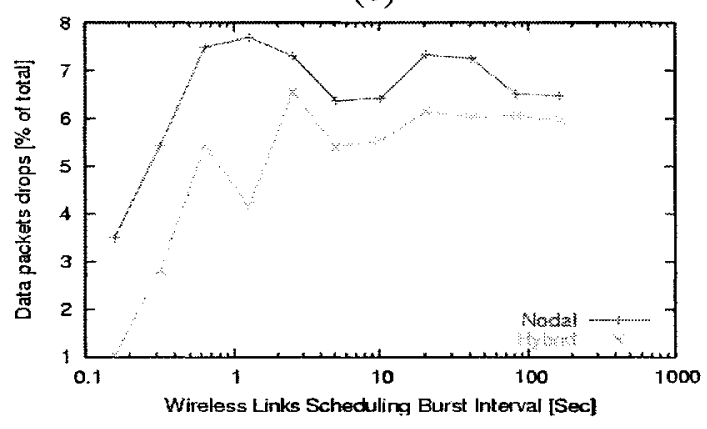

Fig. 6.9 Backpressure model's performances benefits as a function of the congestionduration (a)(b) throughput (c) tandem-queues' data packet drop rate 
Fig. 6.10 shows the performance costs associated with the hybrid flow-control. Low percentage of control packets is observed, compared to the volume of data packet that it supports. Larger average delays are observed at the tandem queues for the reasons explained at 6.2.3. However, the average delays show to converge to the PCF target delay for large 'infinite-burst' intervals. It is achieved for intervals in which the period of dumped bandwidth-swings (and the backpressure reaching quiescence) dominates the average delay.

(a)

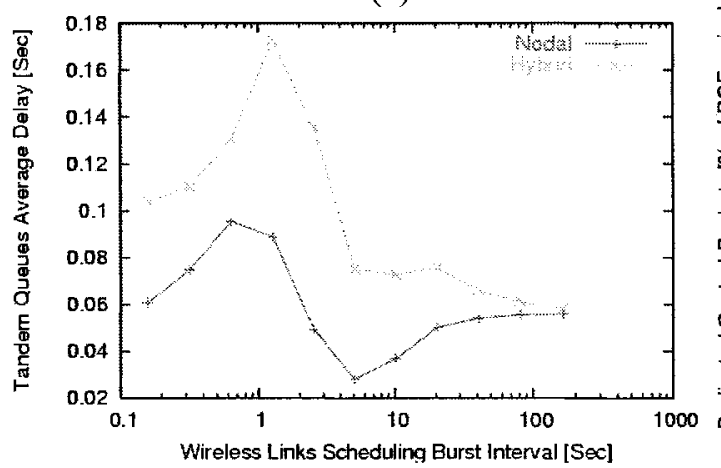

(b)

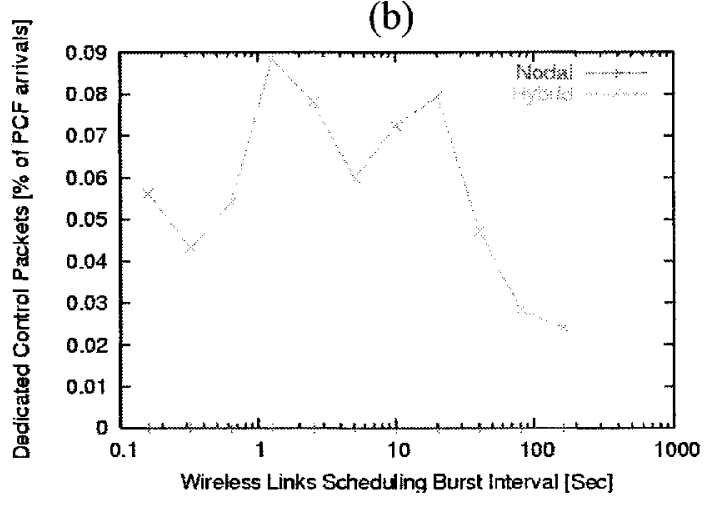

Fig. 6.10 Backpressure model's performances costs as a function of the congestion duration (a) tandem-queues average-delay during 'burst' intervals (b) overhead of control packets

\subsubsection{Comparison with a Nodal Model of Enlarged Buffer}

We compare the hybrid flow-control model with the alternative of nodal model with buffer increased. The nodal model is constructed with a PCF RED queue without backpressure. The PCF buffer size equals the size of the utilized buffers with backpressure, that is, the sum of the PDSN buffer and the PCF buffer. Accordingly, we adjust the PCF's RED thresholds at the nodal model to achieve a target delay that equals the sum of the target delays of the PCF and the PDSN queues.

Fig. 6.11 compares the two models with varied number of active users. RED at the enlarged PCF utilizes the increased buffer-space for accommodating the CDMA-2000 bandwidth swings based large bursts of traffic. However, the incurred overflow-based 
closely clustered drops once traffic loads are heavy results with lower throughput than this of the hybrid mode.

(a)

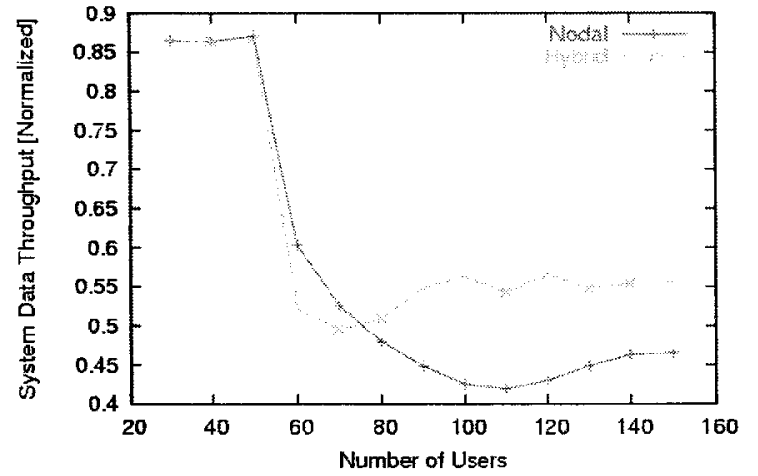

(b)

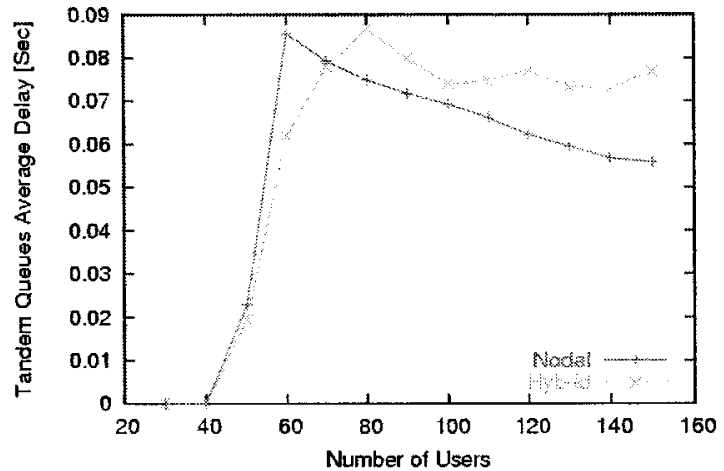

Fig. 6.11 Comparison of backpressure model with nodal model of enlarged buffer size, function of congestion depth: (a) throughput (b) tandem-queues average-delay during 'burst' intervals

Fig. 6.12 compares the two models with varied 'burst' interval. Once the 'burst' interval is long such that the PCF's RED stabilizes the queue to its steady-state target average delay, the average-delay at the tandem queues converge to the target delay of the PCF $(0.5 \cdot R T T)$ at the hybrid model. However, the cost of large incurred average-delays can only persist for the nodal model, and converge to the enlarged PCF's target-delay of $1 \cdot R T T$. The throughput of the hybrid model can only partially surpluses this of the enlarged nodal model. Very short 'burst' intervals present a single traffic burst at the PCF queue, which accommodated with lower drop probabilities at the enlarged nodal model resulting with higher throughputs. Very long 'burst' intervals present higher values of average-delay convergence for the enlarged PCF, similarly resulting with lower drop probabilities and higher throughput. 
(a)
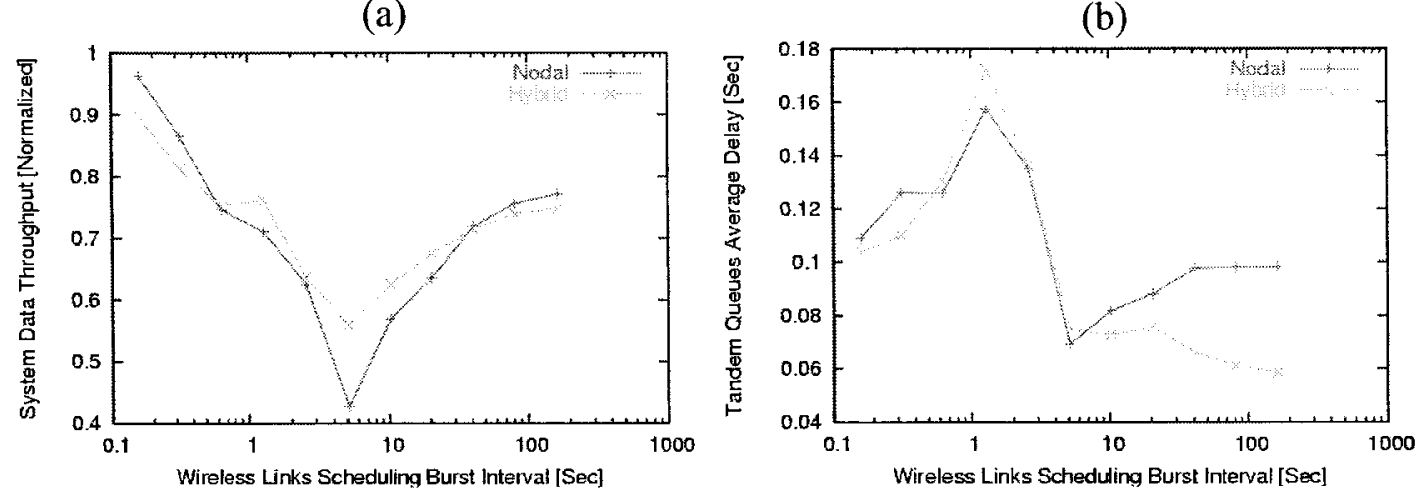

(c)

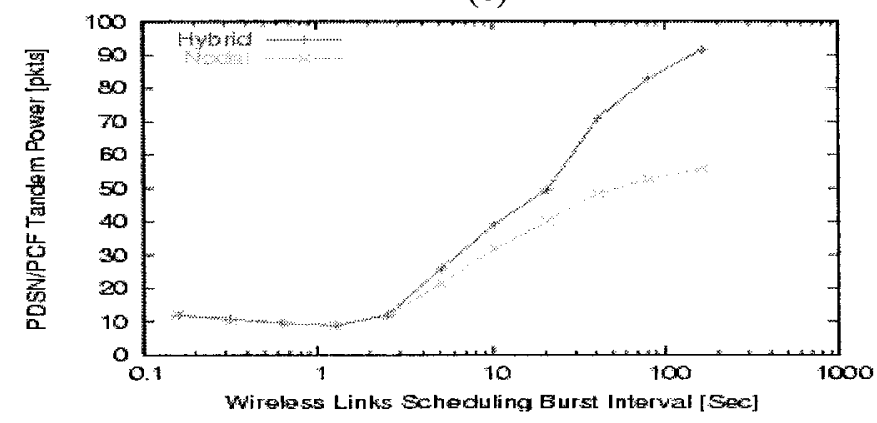

Fig. 6.12 Comparison of backpressure model with nodal model of increased buffer size function of congestion duration: (a) throughput (b) tandem-queues average-delay during 'burst' intervals (c) Tandem power $(=(a) /(b))$

\subsection{Conclusions}

In this chapter we evaluated the 3GPP2 proposal for protecting the bottleneck node by using a backpressure mechanism. It controls the packet transmission rate of the gateway node during excessive congestion at the PCF to protect it from overflow. We propose a backpressure mechanism, the Adaptive-Xoff/Xon, which is capable to adapt to the high variability of the incoming traffic, as common during the bandwidth-swings. The thresholds of the Adaptive-Xoff/Xon at the congested bottleneck node are tuned above the RED's thresholds to cerate a hybrid flow-control model in which the backpressure supplements the nodal RED mechanism.

We analyzed the backpressure model performances, and compare it against a nodal model. The model shows higher system throughput, lower packet drop rate, and absence of overflow-based drops. The observed pertaining costs are: low volume of control 
packets, and higher average delay at the tandem queues. Lower power function values are observed, which suggests that the 3GPP2 proposal for backpressure in CDMA-2000 brings combined performances that are degraded compared to the nodal model.

In the next chapter we explore the possible benefits in harnessing the backpressure mechanism's ability to push congestion towards the feeding node for enhancing the CDMA-2000 network with QoS capabilities. 


\section{QoS Model for Service Differentiation}

The nodes with shared-queues at the CDMA-2000 data network have inherent rate mismatch, which allows for traffic variations and subsequent congestion. It is constructed with a PCF node that is processing limited, and a PDSN feeding node with a superior processing and service rate metrics. The PCF represents the system bottleneck during traffic overload scenarios, whereas the PDSN is largely underutilized. A close-loop backpressure model, an Adaptive-Xoff/Xon, was proposed at chapter 6 to efficiently protect and control the PCF queue by utilizing PDSN spare buffer space during PCF congestion. The CDMA-2000 data network offers a single level of service through the PCF and the PDSN nodes. This cannot differentiate between today's applications' distinct QoS requirements. Differentiated services (DiffServ) have been designed for Internet to provide multiple levels of network services while maintaining a good scalability property through handling aggregates of traffic per hope.

We propose a model for providing service differentiation at the CDMA-2000 data networks. The model aims to provide service differentiations comparable to the traditional DiffServ model. The proposed improvement is in providing those services under the CDMA-2000 structure of tandem nodes with large rate mismatch and a constraint of maintaining the complexity level of the processing limited core node (the PCF). The model uses a combination of backpressure and DiffServ techniques. An instance of the Adaptive-Xoff/Xon backpressure mechanism is used to push congestion conditions from the PCF to the PDSN where superior treatment can be provided to the traffic, hence alleviating the processing consumption at the PCF while still allowing for service differentiation. The lossless PCF shared-queue is further protected with a RED mechanism for failsafe in rare multifunction scenarios. The PDSN is constructed as a DiffServ edge node with multiple physical queues. It is capable of carrying per flow treatment as well as per traffic behavioral-aggregate (BA) handling. Our backpressure enabled QoS model differentiates services in terms of the relative portion of access to 
network shared resources and distinct handling at the multiple RED physical queues. It enables differentiation in terms of the achieved relative throughput, packet loss rate, average delay, and delay jitter, subject to the model's settings.

The questions that this chapter targets are as follows: Is the Adaptive-Xoff/Xon backpressure mechanism capable of conveying packet drops from the bottleneck core node towards the gateway node while allowing for QoS based packet processing there? Can a QoS model for service differentiation, based on the DiffServ architecture, be incorporated in the CDMA-2000 system under the system constraints, and what is the effectiveness of DiffServ model built of multi-queues? In particular, it queries: What are the devised QoS model's performances in handling multiple classes of TCP flows? Can the DiffServ model mitigate the adverse interaction between the multi-transport layers (TCP, and UDP) at the context of a CDMA-2000 system? And, what is the affect of the QoS model on the ACK-compression based bandwidth swings?

We verify our model and demonstrate its robustness with various traffic scenarios and system topologies using simulations under the common NS-2 simulator. We show that the backpressure mechanism performs an efficient congestion control at the PCF node via the control the PDSN transmission rate. In particular, it stabilizes the PCF queue and achieves low occupancy levels and low variability with no packet drops. We show that our architecture is effective in providing bandwidth differentiation using scheduling of multiple physical queues. We further show that our model with the backpressure and single deployed DiffServ node provides throughput, packet drop rate, and average delay preferences.

\subsection{Description of the QoS Model}

The two shared-queues at the system, at the PCF and the PDSN, are of interest for facilitating service differentiation. Therefore, the devised QoS model operates as a special DiffServ domain build of tandem nodes. Fig. 7.1 brings an overview of the QoS model. It is constructed with a DiffServ edge node and a close-loop backpressure. 


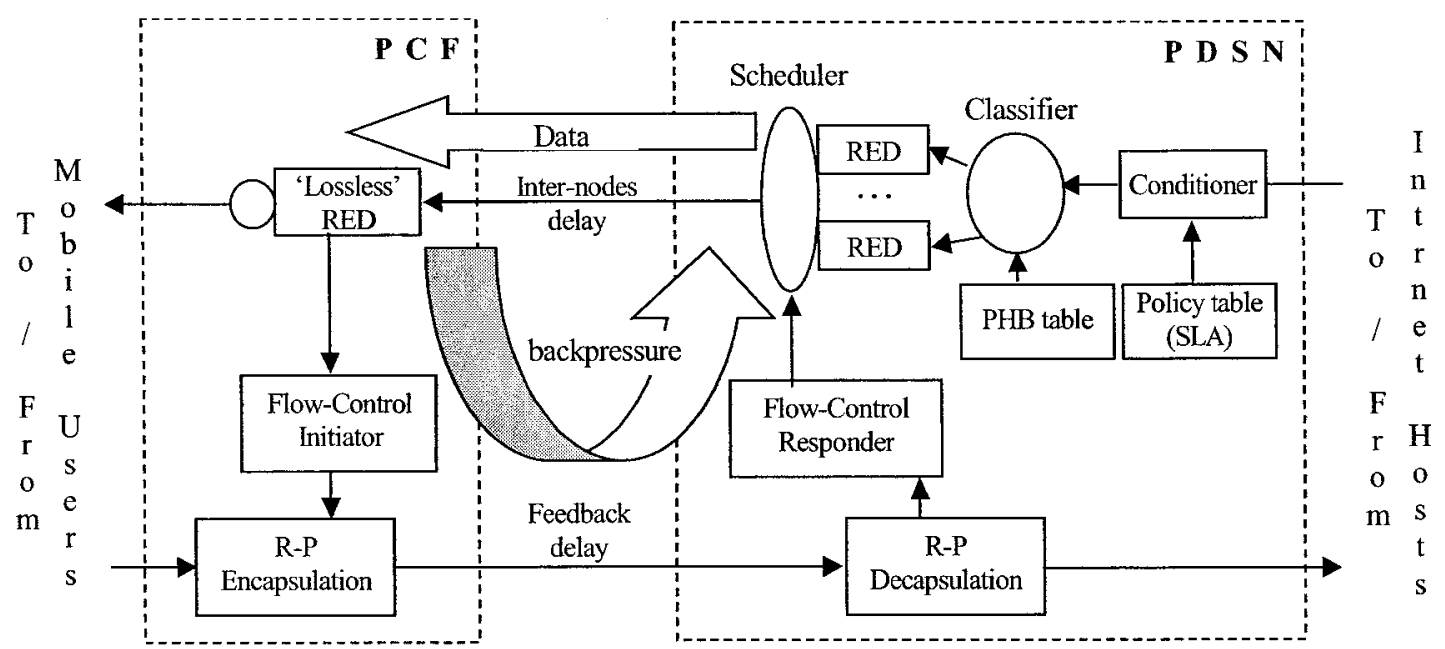

Fig. 7.1 CDMA-2000 DiffServ Architecture with Feedback Flow-Control

The case of CDMA-2000 data system introduces a specific set of constraints and assumptions for our QoS model. As common at $3 \mathrm{G}$ networks, single sided traffic is flowing at the CDMA-2000 forward direction, from the Internet hosts to the mobile users. The PCF has substantially lesser processing and buffering capabilities than at the PDSN. Adding computation and processing load, as well as extending the architectural complexity at the PCF, is undesired. Lastly, backpressure specific constrains and assumptions noted at chapter 6 holds for the QoS model as well.

\subsubsection{The PCF Node}

The PCF node due to the limited processing power does not provide PHB behavior, but is rather designed to push congestion to the PDSN while having little influence on the system level service differentiation. It presents a 'lossless' node with a single queue where the congestion level and average queue-size are controlled by the feeding queue using feedback flow-control. Drops at the PCF are considered as 'noise' drops on top of the differentiated drops at the DiffServ edge node (the superior PDSN). Therefore, packet drops at the PCF are desired to minimize, or better eliminated.

The PCF node employs the congestion detection and notification facilities of the Adaptive-Xoff/Xon backpressure. The Adaptive-Xoff/Xon's a priori average delay (the 
hysteresis-center) and jitter (the hysteresis-size) are essential for our QoS model for providing predicted PCF delays and negligible drop volumes, thus allowing for service differentiations on the CDMA-2000 system level.

A RED AQM with a sufficient queue size is still desired at the PCF for a failsafe in rare cases of non-cooperative PDSN. While this can provide an efficient congestion handling protection fallback for the PCF node, the service differentiation are subject to performance degradation as a function of the ratio between the congestion levels at the PDSN and the PCF (or alternatively their packet drop rates ratio).

\subsubsection{The PDSN}

The PDSN performs the control actions of the backpressure mechanism, as well as carrying out the service differentiation procedures.

Backpressure control actions are performed as a response to incoming control indications from the PCF and related PDSN reaction. The PDSN uses the knowledge of the PCF rate and determine the throttle down value to be a fraction of the PCF service rate. It is chosen to be 0.9 of the PCF service-rate at this paper in order to reduce the PCF queue oscillations and volume of required control packets. The PDSN queue may be congested at times subject to the incoming traffic characteristics, during which intervals traffic class-based differentiation is performed via proper congestion handling.

The QoS architecture at the PDSN is based on a DiffServ edge node model [27]. Traffic entering a network is identified to belong to a certain behavior aggregate according to its service agreement with the network. It is marked with an associated single differentiated services codepoint (DSCP), subject to a possible conditioning. It is then enqueued and forwarded according to the per-hop behavior associated with the DS codepoint by using multiple physical queues and scheduling entity. The key components of the deployed differentiated services components at the PDSN are explained below.

The SLA specifies packets marking rules allowing the classification to different behavioral aggregates (BAs), and may also specify traffic profiles for the use of the 
traffic conditioner. In this paper we are interested in investigating service differentiation provided by the combination of the backpressure, multiple physical queue at the PDSN, and the scheduling procedures. Therefore, the conditioning functionality is bounded to adjusting the arrivals' DS codepoints to their initial DS codepoint at the SLA.

The classifier is responsible for forwarding packets to their class-based queue for further behavioral aggregate treatment. It acts upon the conditioner assigned DS codepoints and uses a PHB table to map a code point to a particular physical queue.

The DiffServ architecture at the PDSN supports multiple classes of traffic, where packets belonging to a single class of traffic are enqueued into one corresponding physical RED queue. Each of the queues is independently tuned to achieve performance specific to the traffic class (or BA), such as: drop-rate, delay jitter, and average delay to throughput tradeoff. The QoS architectural depicted at Fig.2 can be constructed with number of physical queues up to the supported number by the DSCP field [28].

A scheduler is instrumented in order to assign relative portions of the out-link bandwidth to the various behavioral aggregates. It differentiates the link bandwidth using pre-programmed fixed weights that are associated with the behavioral aggregates. The deployed scheduler for the study in this chapter follows the weighted round robin (WRR) scheme. The rate adjustments of the backpressure control the scheduler's aggregated rate. This way the ratio of bandwidth sharing between the classes is maintain for variable levels of congestion, excessive delays per class are avoided (i.e., live-locks), and traffic classes are guaranteed with at least their assigned bandwidth portion during congestion.

\subsubsection{Parameters Setting}

This section presents considerations in setting the RED queues and system parameters. The following presents for simplicity the tune up recommendation for RED for achieving equal average-delays at the PDSN queues during congestion. These basic concepts can then be easily manipolated to create delay differenting system. The effectiveness of the proposed tunings is demontrated by our simulation results at 7.2. 
The multiple RED queues at the PDSN maintain their settings in accordance with the recommendations at [6][10]. In the context of our QoS model, the service rate of a specific PDSN queue is subject to the backpressure operation and bandwidth differentiated allocation enforced by the scheduler. The PDSN queues are only congested while responding to backpressure indications from a congested PCF. The maximum rate of the PDSN out-link rate is considered as multiples of the rate of PCF out link, however the effective PDSN out-link rate during PCF congestion matches this of the PCF rate on the average due to the backpressure operation. This is achieved while the PCF queue occupancy is stabilized to incur no (or negligible) drops. Furthermore, the presence of a bandwidth scheduler that arbitrates between the PDSN's queues enforces additional rate division of the PDSN out-link effective rate, as viewed by a particular queue at the PDSN. Hence, the affective link rate for such a particular queue at a multi-queues architecture with bandwidth scheduling at the PDSN is:

$$
B W_{e f f}^{Q_{-} i}=B W_{P C F} \cdot\left(W R R_{\text {weight }}^{Q_{-i}} / \sum_{k=1}^{n} W R R_{\text {weight }}^{Q_{-k}}\right)
$$

where $n$ physical queues are available at the PDSN, $B W_{P C F}$ is the PCF out-link rate, and $W R R_{\text {wieght }}^{Q_{-}^{i}}$ is the WRR weight associated with physical queue $i$ at the PDSN. For physical queue $i$, the desired target queue fill $q_{t \text { arget }}^{Q_{-} i}$ is determined to allow for a target average delay $D_{t \text { arget }}^{Q i}$ during congestion:

$$
q_{t \text { arget }}^{Q_{-} i}=D_{t \text { arget }}^{Q_{-} i} / B W_{e f f}^{Q_{-} i}
$$

The RED queue lower and upper thresholds, and buffer limit can now be rewritten as:

$$
\begin{aligned}
& t h_{\min }^{Q_{-i}}=2 \cdot q_{\text {targ et }}^{Q_{-i}} /\left(1+t h s_{\text {ratio }}^{Q_{-i}^{i}}\right) \\
& t h_{\max }^{Q_{-} i}=3 \cdot t h_{\min }^{Q_{-i}^{i}} \text { (i.e., } t h s_{\text {ratio }}^{Q_{-} i}=t h_{\min }^{Q_{-}^{i}} / t h_{\max }^{Q_{-}^{i}}=3 \text { ) } \\
& \operatorname{size}_{\text {buffer }}^{Q \rightarrow i} \geq 2 \cdot t h_{\max }^{Q-i}
\end{aligned}
$$


Lastly, the averaging weight for each physical queue at the PDSN is derived by assigning $B W_{\text {eff }}^{Q}{ }^{i}$ to Eq. (2.7):

$$
w_{q}^{Q_{-} i}=1-\exp \left(-1 /\left(N \cdot R T T \cdot B W_{e f f}^{Q_{-i}}\right)\right)
$$

where the averaging-factor $N$ is the desired number of RTTs for the RED to average the queue-size to $63 \%$ of its instantaneous queue-size, and the effective bandwidth of queue $i$ at the PDSN, $B W_{e f f}^{Q_{-}{ }^{i}}$, is in [packets/second]. Note that the above equations consider the worst-case service rate per queue for an assumed PDSN with concurrent congested queues. This is done to assure minimum set of delivered performances for a specific behavioral aggregate.

The backpressure hysteresis-center is required to be tuned to be below the RED lower threshold, thus avoiding packets drops at the PCF for most overload scenarios. The backpressure hysteresis-size is required to be tuned with low values to keep the backpressure based occupancy variations at the PCF below RED lower threshold, and on the other hand avoiding queue underutilization for low hysteresis-size values due the backpressure-based occupancy variations.

With the backpressure in place, the desired average-delay during congestion for a specific traffic behavioral aggregate should now consider the PCF and the PDSN delays. For instance, a desired $0.65 *$ RTT delay for a specific traffic class may be constructed with $0.15^{*}$ RTT at the PCF and $0.5^{*}$ RTT per queue at the PDSN. The lower target-queue size is required to be set at the PCF. It is required for limiting the effect of the PCF single delay value on the multi traffic aggregates. It is also for increasing the system's utilization since for persistent traffic overloads the PCF is expected to become empty only after the feeding queue (PDSN) become empty.

\subsection{Simulations Results and Discussion}

In this section we evaluate the QoS model using simulations. $\mathrm{A} \mathrm{C}++$ model is constructed and exercised under the popular NS-2 simulator [33]. We explore wide range of traffic 
and system scenarios to investigate the sensitivity of our devised QoS model to the system parameters, verify its robustness, and assess the achieved differentiated services.

\subsubsection{Simulation Setup}

This section describes the default traffic and system setups in addition to the described at chapter 3 . These setups are used in the subsequent performance evaluation case studies. In the various tests we may vary one or more of the setup's parameters.

The setup of performance evaluation simulations follows few assumptions and constraints. First, flows within a single physical queue at the PDSN are identical, hence they are considered to require an equal profile. Second, the number of active connections for all traffic classes is set to be equal to allow for the service grade between traffic classes to be experienced on the basis of each flow.

Three traffic sources models are used in the simulation: infinite FTP source over TCP, interactive finite FTP source with 'thinking' intervals over TCP, and CBR source with random inter packets intervals. The CWND of all TCP source of $10 \mathrm{pkts}$ is large such that it allows the DiffServ system to limit the sources' rates, and monitor the system response, rather than differentiating the sources rates in the first place. See chapter 3 for details.

The CDMA-2000 system is constructed with a single shared queue at the PCF, and a DiffServ architecture with 2 physical queues at the PDSN. Traffic destined to queues number 0 is marked with DSCP of 0 , whereas traffic destined to queue number 1 is marked with DSCP of 4 . At each of the two traffic classes, 50 Internet-hosts communicating with 50 mobile stations, each using a unicast single-sided traffic flowing from the Internet-hosts towards the mobile-stations at the CDMA-2000 forward direction (see Fig. 3.1). Each of the PDSN queues' target occupancy follows Eq.(7.2). The buffers at the PDSN queues follow Eq.(7.5) derived for WRR weights of 1 and 2 to be 100KB and 50KB, respectively. The PDSN queues are tuned to comparable characteristics by means of average delay and jitter using Eq.(7.3), Eq.(7.4), and the equality of Eq.(7.5). 
That is, the target average delay of each queue is equal, regardless of its assigned WRR weight.

The SCH assignment procedure interleaves the SCHs allocation (and de-allocations) between the DS groups to allow comparable bandwidth allocation and de-allocation intervals for the different traffic classes.

The backpressure set-up follows the directions at chapter 6 and at 7.1.3. In our simulations the hyteresis-center is tuned to $15 \%$ of the PCF queue, which is below the RED lower threshold. The hyteresis-size is tuned to $10 \%$ of the PCF queue allowing low occupancy variations at the PCF shared queue while maintaining a large enough hysteresis to reduce the volume of control packets.

\subsubsection{Temporal Queue and System Dynamics}

This section presents the QoS model's temporal dynamics. At the simulations, the burst interval for this test scenario was set to 20 seconds to clearly demonstrate the queuing dynamics. The results are shown in Fig. 7.2 and interpreted below.

Throughput differentiation is shown by Fig. 7.2a. The effect of the ACK-compression based bandwidth swings are observed at the figure. The sum of the two traffic-classes throughputs is shown to increase and approach the PCF bottleneck bandwidth as the queues oscillations dump towards the end of the burst interval. Packet drop-based differentiation is shown by Fig. 7.2b. Flows that traverse queue number 0 with the assigned smaller portion of the bandwidth suffer from higher packet drop volume compared to flows with higher priority that use queue 1 . The backpressure is observed at Fig. 7.2c to control the PCF queues fill below the PCF RED lower threshold, hence avoiding PCF drops and incurring low queuing delays. 
(a)

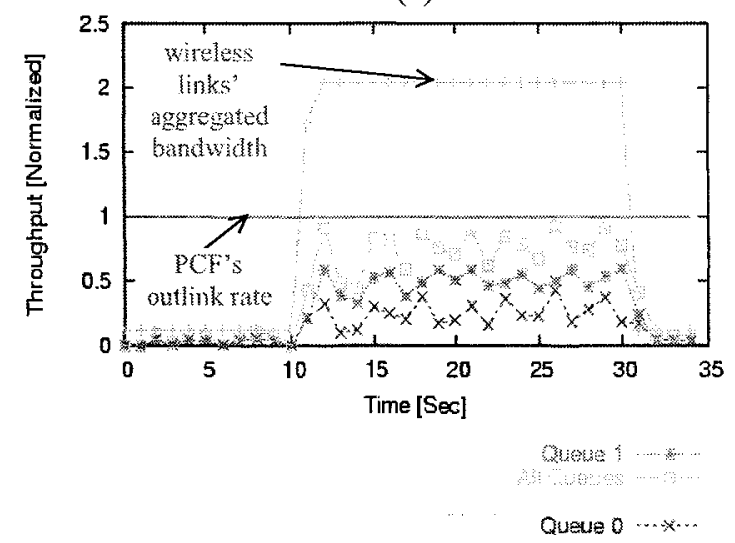

(b)

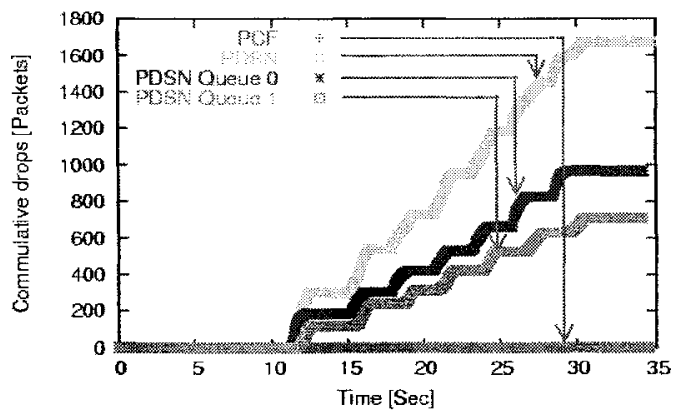

(c)

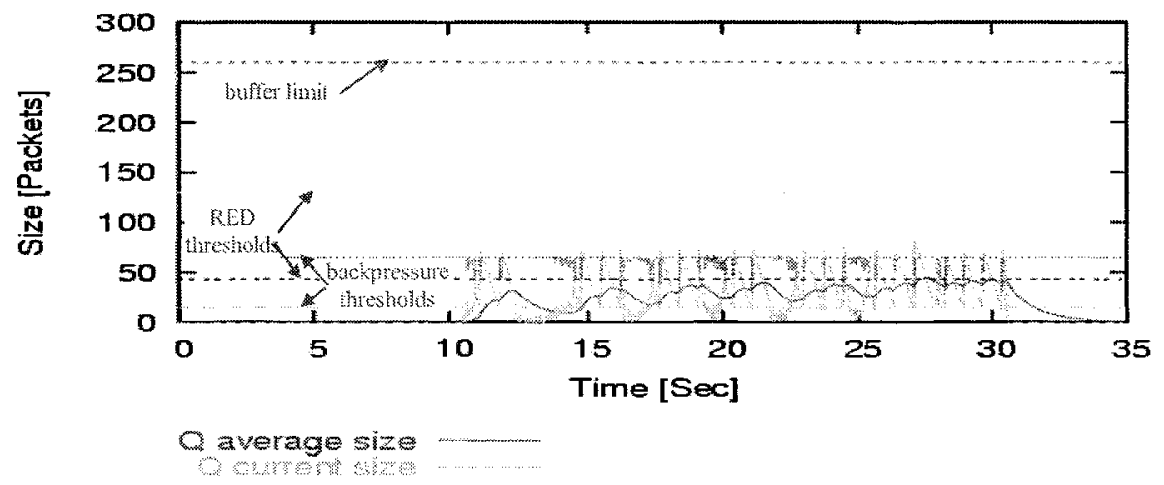

Fig. 7.2 Temporal PCF and PDSN queuing and backpressure dynamics (a) wireless link bandwidth and throughput (b) PCF and PDSN RED queues commutative drops (c) PCF RED queues occupancy dynamics

\subsubsection{Effect of Congestion Depth}

We evaluate the QoS model with number of connections ranging from 10 to 170 active connections. This represents varied PCF overload levels and consequent congestion depths (defined as the ratio between the PCF ingress and egress rates). The results are shown in Fig. 7.3. 
(a)

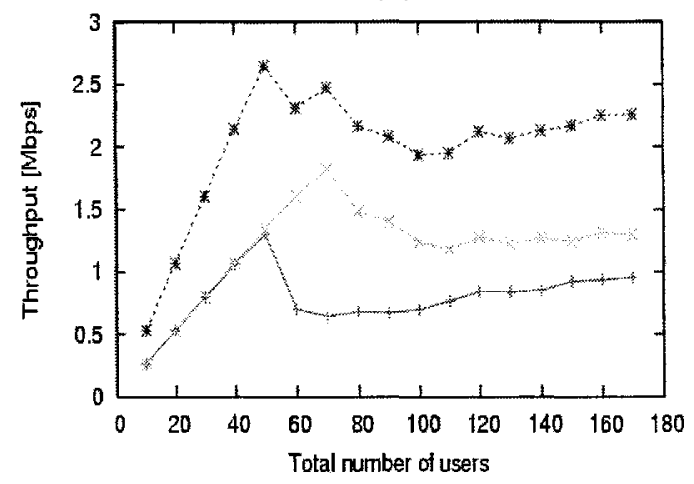

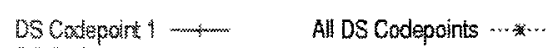
mont?

(c)

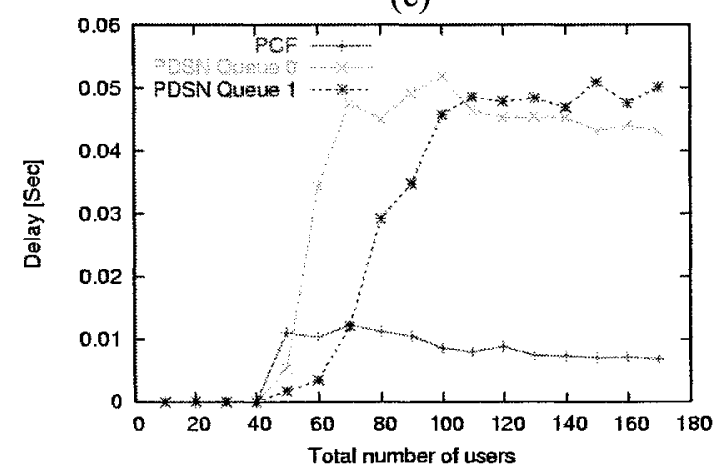

(e)

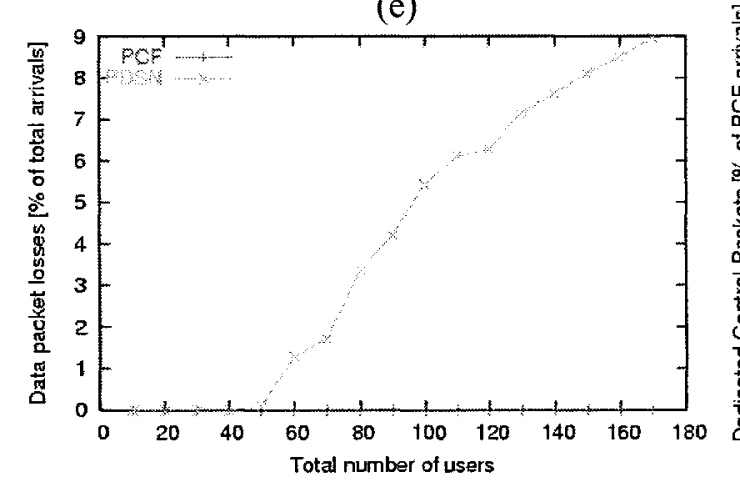

(b)

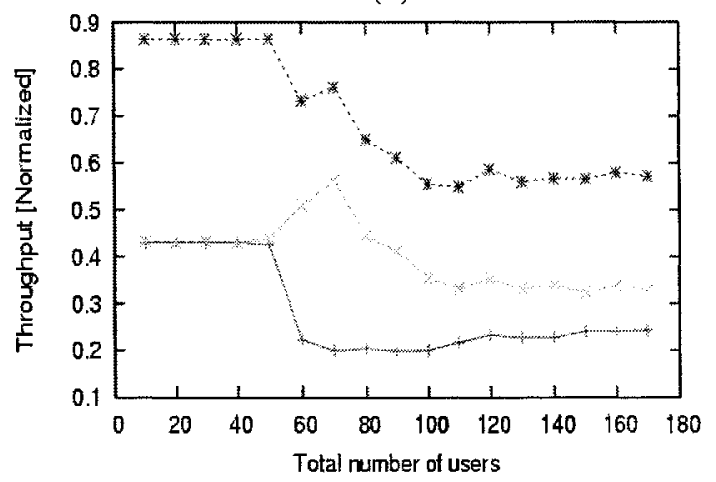

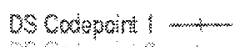

Al DS Codepoints …….

(d)

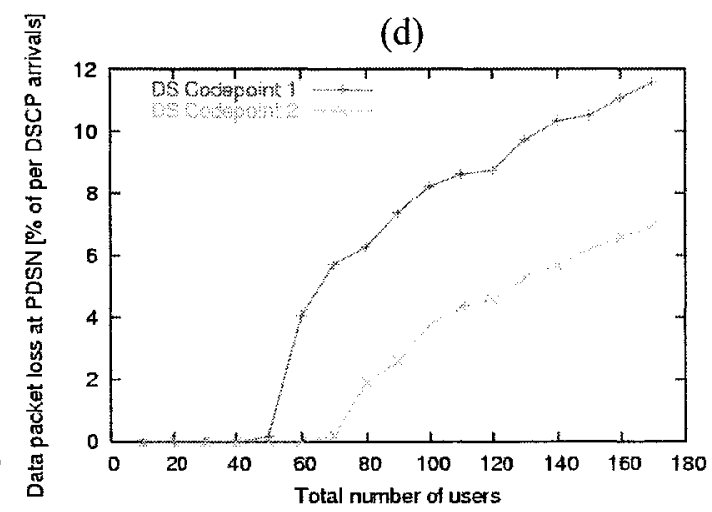

(f)

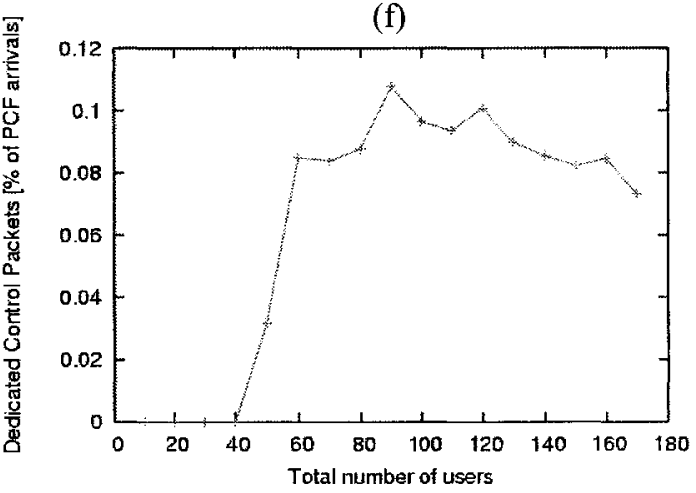

Fig. 7.3 QoS model performance as a function of the congestion depth (a)(b) system and class-based throughputs (c) average delay during congestion (d) percentage of data packet loss volume at the PDSN per traffic class (e) Data packet loss volume distribution between the PCF and the PDSN (f) backpressure control packet overhead

The throughput differentiation is shown in Fig. 7.3a,b. The congestion built-ups and congestion collapses of both queues at the PDSN are clearly observed. The system throughput cannot reach the system bottleneck bandwidth due to the delay intervals of the 
finite-burst mode scheduling of the wireless links. Throughput differentiation is clearly observed. However, for higher traffic load throughput differentiation lower than the WRR assigned weights is observed. This is since the halving of the TCP senders' congestion window at the advent of a packet loss affects flows with higher assured rate more than the ones with lower assured rate, as the formers operate with larger windows. The ACK-compression based excessive drop rates results with such repeated effects.

The packet drops differentiation presented by Fig. 7.3d. The percentages of dropped data packets at the RED queues show an achieved drop differentiation by the QoS model, subject to the setups.

The average delays at all PDSN queues are observed to converge to the target queue fill once the traffic load is sufficient to fully utilize and congest the queue (Fig. 7.3c). The larger the bandwidth share assigned to the queue, the greater the number of user to congest the queue such that the target average delay is reached. Adequate backpressure control of the PCF with no PCF drops, low and sustainable average delays at the PCF, and low backpressure indication packets overhead are observed (Fig. 7.3c,f)

\subsubsection{Effect of Congestion Duration}

We evaluate the QoS model with various congestion durations, using a range of $\mathrm{SCH}$ allocation intervals. We use a set of values to represent both 'finite-burst' and 'infiniteburst' wireless link scheduling modes (see chapter 2.1). The values set used for the burst time interval is: $0.16,0.32,0.64,1.28,2.56,5.12,10.24,20.48,40.96$, and 81.92 [seconds], which are presented with logarithmical $x$ axis at in Fig. 7.4. The first 6 values $(0.16$ - 5.12) are taken from the CDMA-2000 standard for 'finite-burst', and the rest are arbitrarily chosen in the 'infinite-burst' range.

Throughput differentiation is demonstrated in Fig. 7.4a,b. The results show persistent throughput differentiation for the BA's. The observed throughput differentiation is lower than the programmed WRR values in general. This is as a result from the interaction between the WRR scheduler operation, the large burstiness of the incoming traffic due to 
the CDMA-2000 finite burst mode, and the TCP congestion window halving, as explained in section 7.2.3. As aforementioned at the chapter 3 , the throughput measurement is normalized with the instantaneous bottleneck rate. This allows us to show the measured throughput as a fraction of the maximum offered bandwidth by the system, independently of metrics such as the burst and delay intervals lengths.

Consecutively, once the burst interval is small such that it ends before the aforementioned overflow-based drops, the normalized throughput is maximized. Similarly, the normalized throughput is maximized once the burst interval is large such that the first TCP senders prolong timeout-based recovery is overshadowed by the packet volume traversing the queue with moderate drop-rates. Clearly, a gradual increase of the throughput as the burst interval increase is observed, which is expected due to increased aggregated period with higher wireless links bandwidths at the system throughout the simulation.

Comparable delays cross traffic classes that converge to their pre-programmed target occupancy for the higher half of the burst intervals are observed at the PDSN queues (Fig. 7.4c). This reflects our RED queues setting for matching delays for BA's in our scenarios. Small burst intervals incur larger measured average delay values, and in particular for the queue with the lower assigned bandwidth portion. This is subject to the measurement method that considers only delays at the queue during the 'burst' intervals. Thus the initial ACK-compression based large burst of traffic accommodated in the RED queue at each 'burst' has a dominant effect on the average delay readings for small burst intervals. The drop percentage shows persistent drop differentiation for all burst intervals, with variations for similar reasons as above. 
(a)

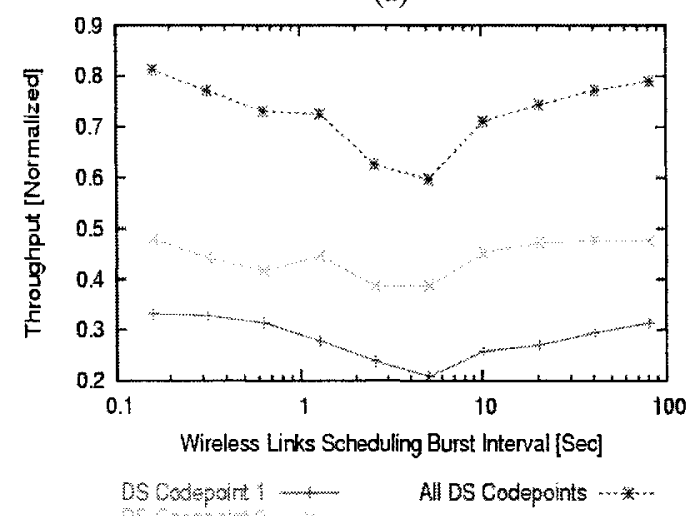

(c)

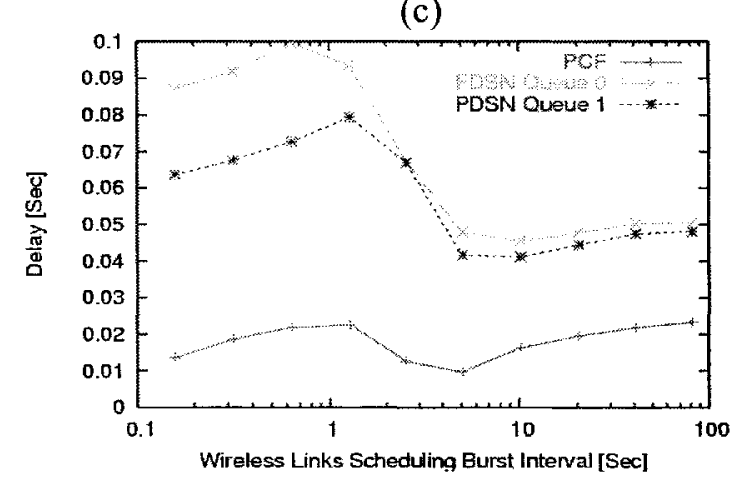

(e)

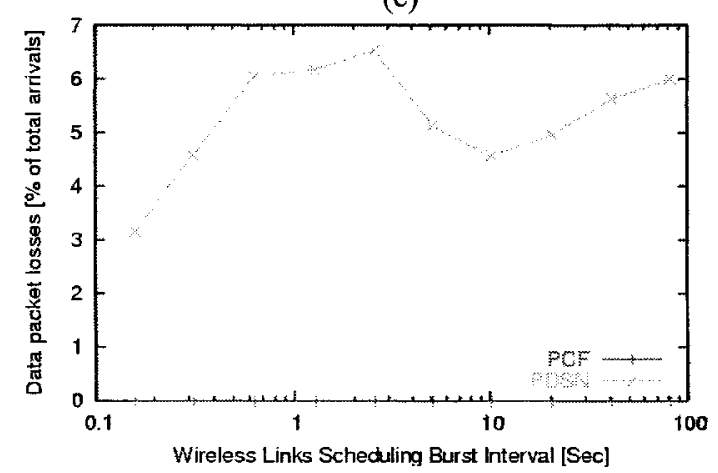

(b)

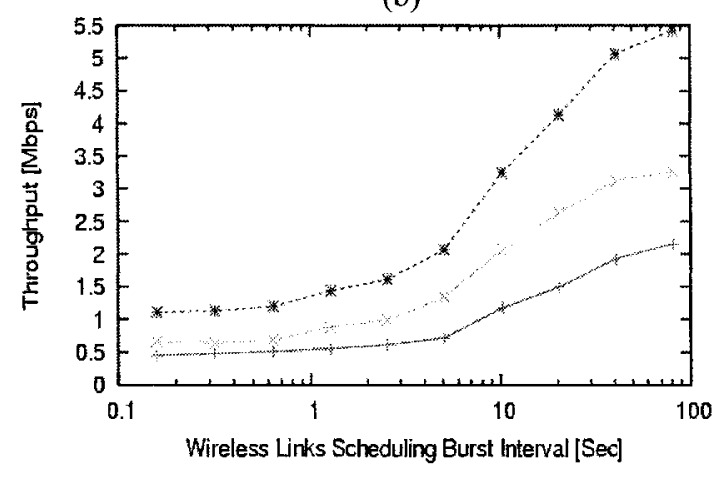

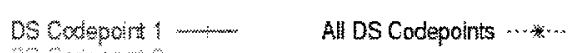

(d)

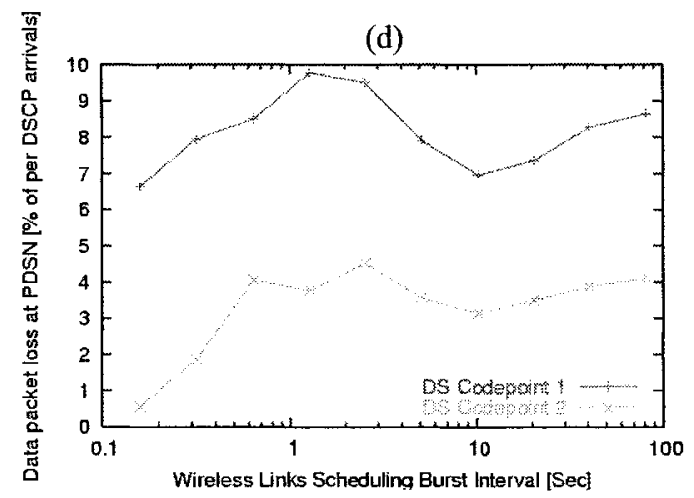

(f)

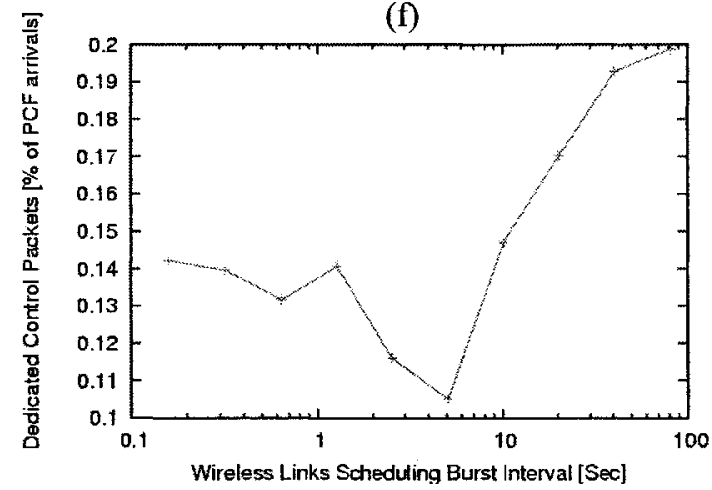

Fig. 7.4 QoS model performance as a function of the congestion duration (a)(b) system and class-based throughputs (c) average delay during congestion (d) percentage of data packet loss volume at the PDSN per traffic class (e) Data packet loss volume distribution between the PCF and the PDSN (f) backpressure control packet overhead

The control of the backpressure mechanism over the PCF queue fill show an adequate operation such that no data packets are dropped at the PCF with all burst intervals, while as aforementioned the PCF queue is stabilized to low delay values (Fig. 7.4c). Moreover, 
the control overhead is shown to be small compared to the traffic volume that it supports, as presented by the control overhead as a percentage of the PCF arrivals at Fig. 7.4d.

\subsubsection{Effect of Backpressure Close-Loop Delay}

The backpressure close-loop delay consists of the PDSN to and from the PCF internodes propagation delays and the control packets transmission time interval, as formalized in Eq.6.1. Fig. 7.5 shows the QoS model performance as a function of the pipe delay between the PDSN and the PCF, which represents half of the control-loop propagation delay. We vary the propagation delay between 0 to 10 Seconds, which represents a wide range of up to $20 \%$ of the flows' RTT (excluding queuing delays).

The simulation results of the QoS model show sustainable service differentiation throughout the tested range of the close-loop delay. The throughput measurements show a sustainable service differentiation in Fig. 7.5a. The throughput differentiation closely follows the WRR assigned relative bandwidth differentiation, but slightly less due to larger ACK-compression and RED effects on behavioral aggregate with the higher assigned bandwidth, as aforementioned in section 7.2.3. All PDSN queues' average delays during congestion periods converge to their pre-programmed target delay at Fig. $7.5 b$.

The PCF average delay is low, as pre-programmed and controlled by the backpressure mechanism. Larger close-loop delays introduce slightly larger delays at the PCF due to larger bursts of traffic at the PCF ingress. The data packet drops in Fig. 7.5c shows dropbased service differentiation for the traffic classes.

The backpressure mechanism shows a proper operation with low PCF average delay (Fig. 7.5b), no drops at the PCF (Fig. 7.5d), and low control overhead percentage (Fig. 7.5e). The control-packets' overhead has an increase trend with the loop delay increases due to the backpressure's upper threshold greater adaptation, which results with lower effective gap to the backpressure's lower threshold. 
(a)
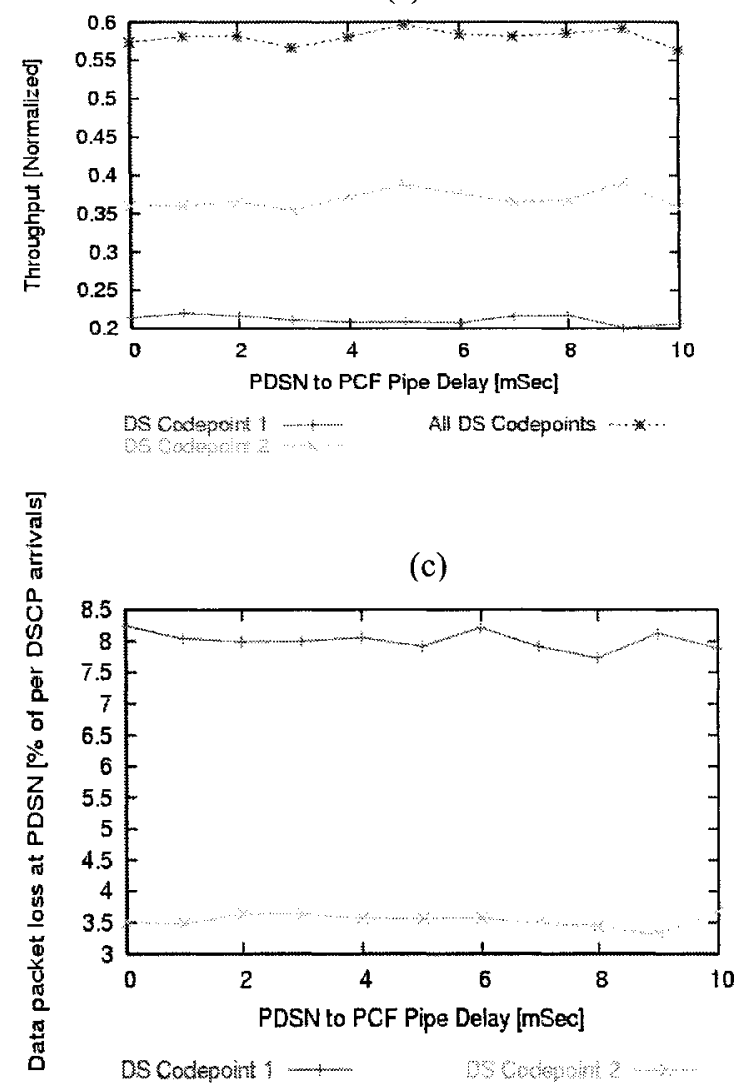

(b)

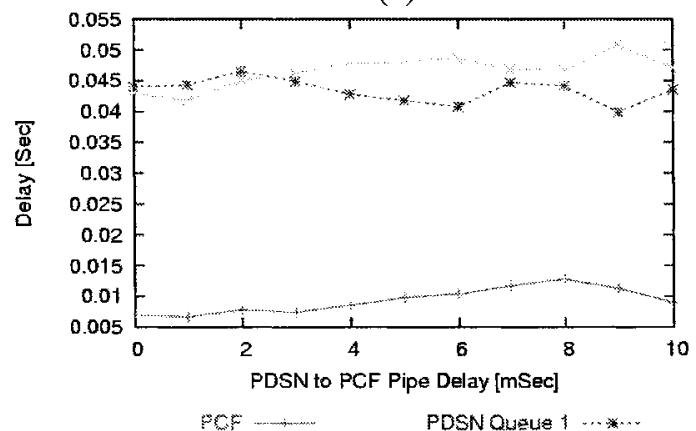

Pro

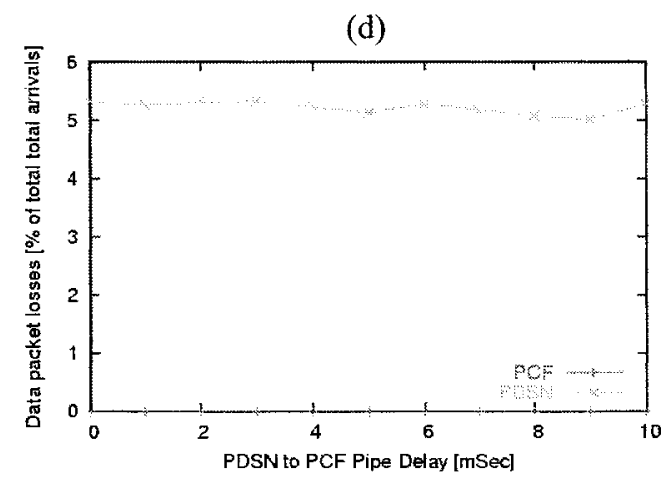

(e)

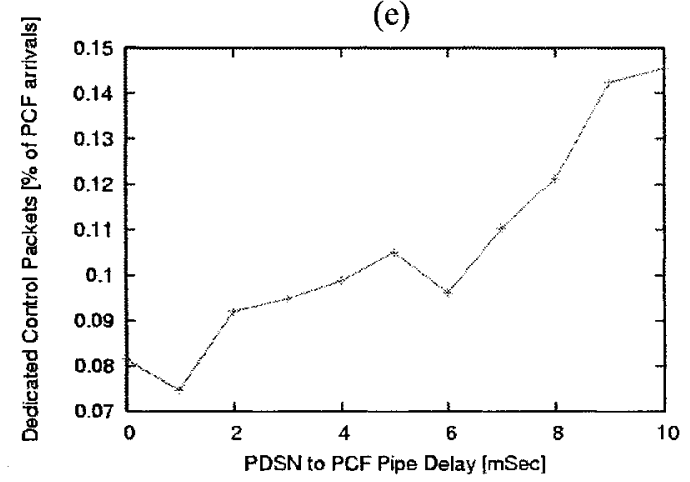

Fig. 7.5 QoS model performance as a function of the backpressure close-loop delay (a) system and class-based throughputs (b) average delay during congestion (c) percentage of data packet loss volume at the PDSN per traffic class (d) Data packet loss volume distribution between the PCF and the PDSN (e) backpressure control packet overhead

\subsubsection{Effect of Bursty Traffic}

In this section use the interactive traffic sources model to generate a more bursty traffic.

The PCF bottleneck service-rate is set to $4 \mathrm{Mbps}$ since the interactive sources introduces a 
lower offered load to the system on the average compared to an infinite backlogged traffic source. The sources' file size for a single transfer is set to 5 MSS (i.e., 2880 Bytes).

The simulation results of the QoS model show service differentiation closely comparable to the infinite FTP case. The system throughput at Fig. 7.6a,b show sustainable differentiation once the offered load to the system exceeds the PCF bottleneck rate. Higher levels of achieved throughput, both for per class as well as system-wide, compared to the infinite sources case are observed. This is due to a less persistent ACKcompression built-up at the wireless links' queues with interactive traffic. The increase of the absolute throughput values at Fig. 7.6a after the two PDSN queues are congested results from the fixed CDMA-2000 wireless links scheduling burst rise time interval for all the simulations iterations. A larger number of users with the same burst rise interval cause the aggregated wireless links to sooner exceed the PCF bottleneck, hence larger offered bandwidth by the system. The measurement of the normalized throughput compensates these differences to allow a correct throughput observation as it uses the system offered bandwidth for the normalization (similar effect can also be observed at Fig. 7.3a,b at section 7.2.3). The average delay (Fig. 7.6c) at each of the PDSN queues reaches its target queue fill once the traffic load is sufficient to fully utilize and congest the class queue. The RED queue design for allowing traffic bursts at the queue results with slightly higher average delays at the queues for the bursty traffic compared to the less bursty traffic at 7.2.3. The percentage of dropped data packets by the RED queues shows that drops-based differentiation achieved by the QoS model under equal number of users.

The backpressure control dynamics are shown at Fig. 7.6e,f. The PCF ingress experiences large traffic burstiness. The control of the backpressure mechanism over the PCF queue fill show an adequate operation such that no data packets are dropped at the PCF. The PCF delay is shown to converge to low delays level, significantly lower than the PDSN queues. The control overhead at Fig. $7.6 \mathrm{f}$ is shown to be small compared to the 
traffic volume that it supports, whereas here too it is slightly larger than the control overhead at 7.2.3.

(a)

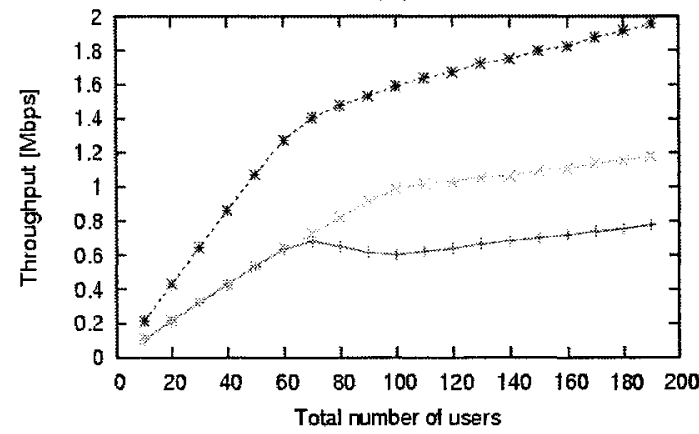

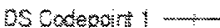

All DS Codepoints $\cdots \cdots$

(c)

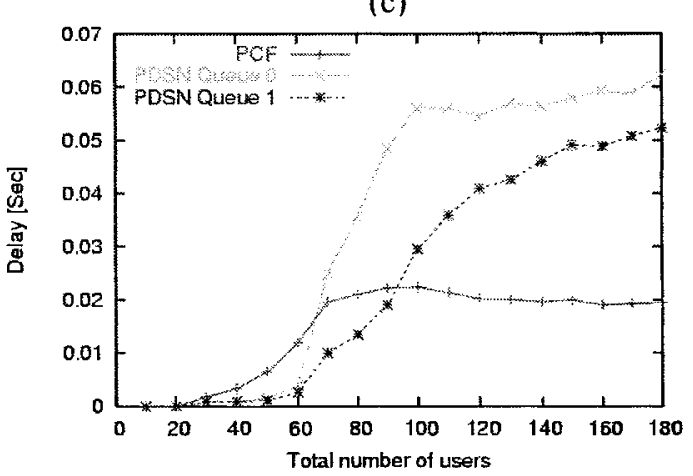

(e)

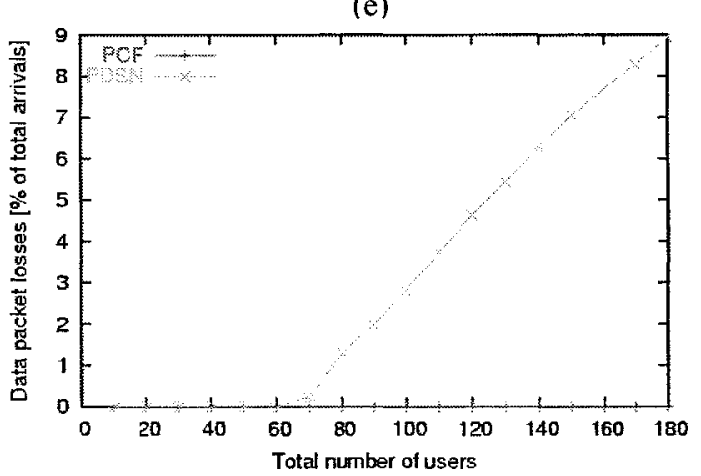

(b)

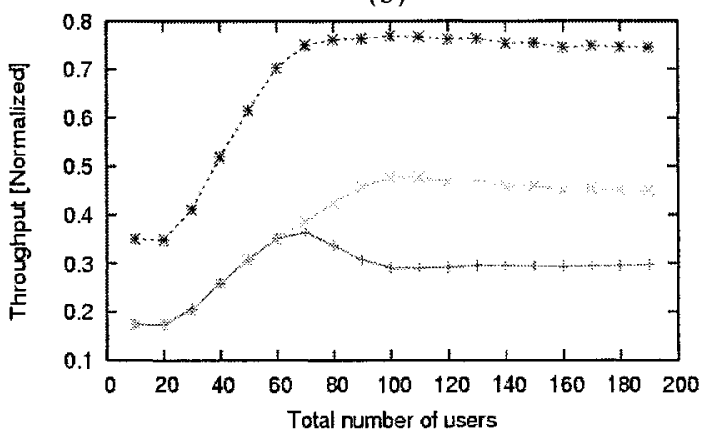

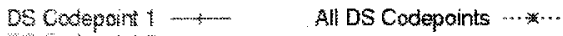

(d)

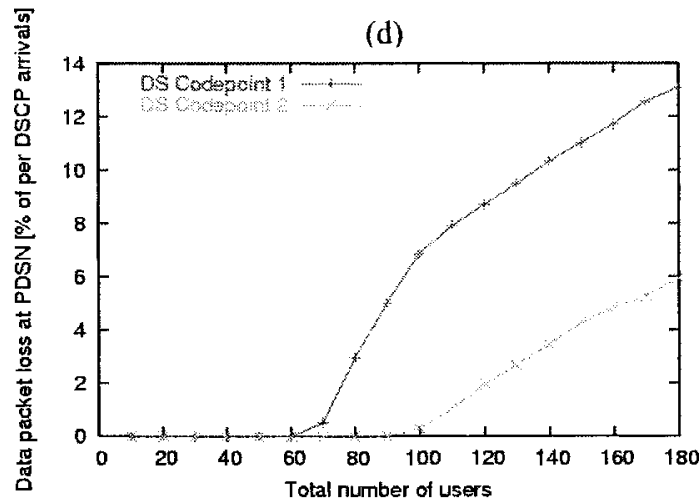

(f)

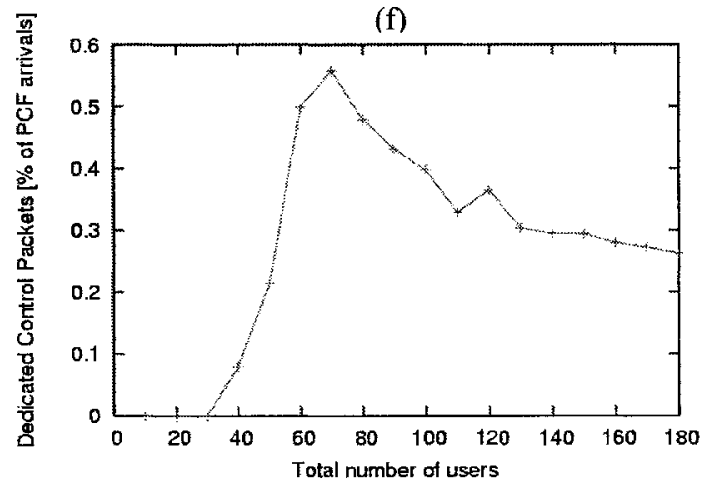

Fig. 7.6 QoS model performance as a function of the backpressure close-loop delay (a) system and class-based throughputs (b) average delay during congestion (c) percentage of

data packet loss volume at the PDSN per traffic class (d) Data packet loss volume distribution between the PCF and the PDSN (e) backpressure control packet overhead 


\subsubsection{Effect of Multiple Transport Layers}

In this section we evaluate the affect of a mix of transport layers, namely TCP and UDP, on the backpressure enabled QoS model performances. In order to closely examine the cross effect between the transport layers enforced by our QoS model, we tune the system to equal bandwidth allocation by the WRR, and subsequent equal PDSN RED queues setups following Eq.(7.1). The TCP uses the infinite greedy FTP sources type. The rate of the CBR sources over the UDP connections are being varied up to the maximum assigned wireless link rate for each flow (i.e., $\mathrm{FCH}+\mathrm{SCH}$ ). The UDP rate (the $x$ axis) is described as multiples of the TCP-friendly rate [9], which calculated for this scenario as the PCF rate (i.e., the bottleneck during burst interval) divided by the total number of active connections in the system.

The throughput result of the QoS model compared to a system with a single service level and with no backpressure is shown in Fig. 7.7. With our QoS model the TCP and the UDP aggregated throughputs show to converge to fixed values, whereas with the single class handling the UDP throughput keep rising and driving the TCP throughput to lower values. The TCP and UDP convergence to unequal throughput values despite the equal WRR weight is legitimate due to the dynamic utilization of bandwidth by the WRR during the ACK-compression based prolong TCP connections back-off. Our QoS model provides the TCP with at-least its fare shared of the bandwidth independently of the concurrent UDP traffic loads as soon as it is required for the recovery phases.

(a)

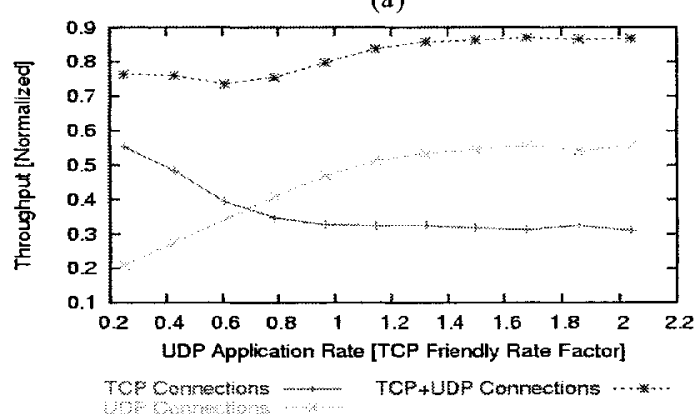

(b)

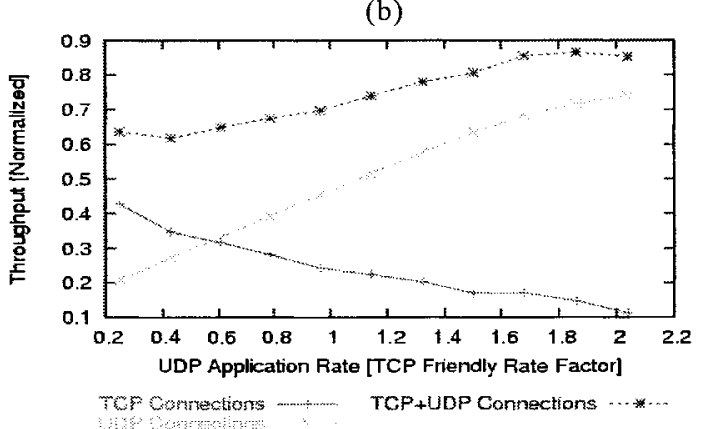

Fig. 7.7 QoS model's throughput as a function of the UDP application rate (a) PDSN with 2 distinct queues (b) nodal model of a single service level 
Fig. 7.8 stresses the QoS model performances with dual transport layers. At Fig. 7.8a once the UDP connections increase their rates, and in particular each surpluses the TCP friendly rate, the TCP queue delay grows up to its predefined target delay level $(50 \mathrm{msec})$. At high UDP loads, the PDSN RED queue is unable to maintain its target queue size for UDP traffic due to its large burstiness and unresponsiveness to congestion notifications. Fig. 7.8b shows low TCP drop-rates with light UDP loads and a constant drop rate for the TCP flows for heavy and variable UDP loads, as desired using the WRR property of bandwidth distribution.
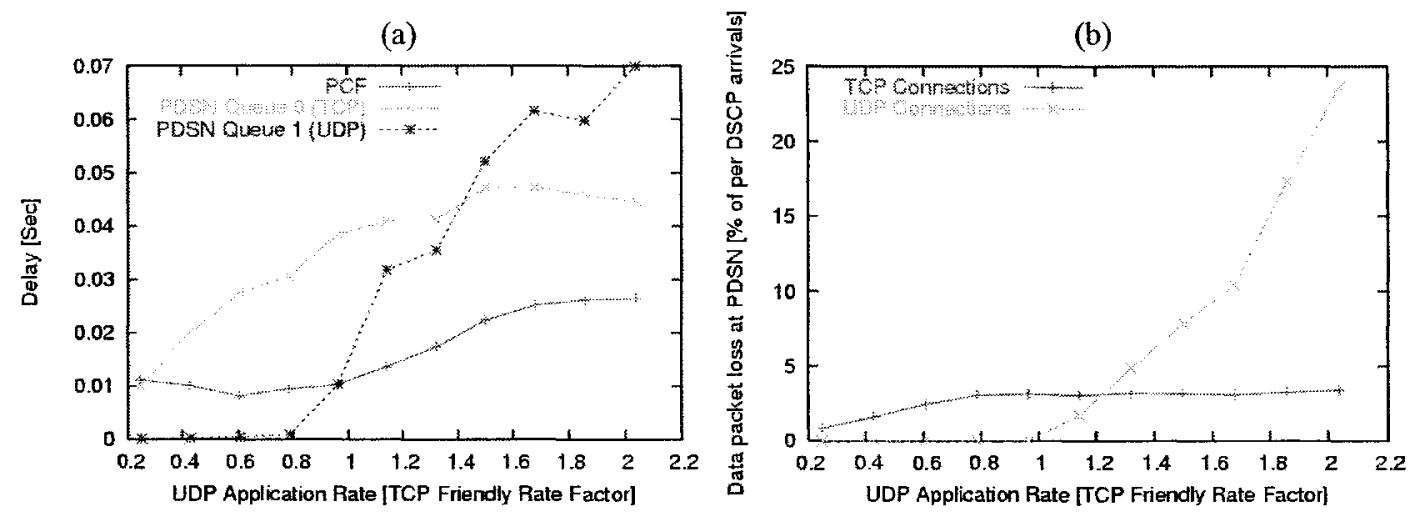

(c)
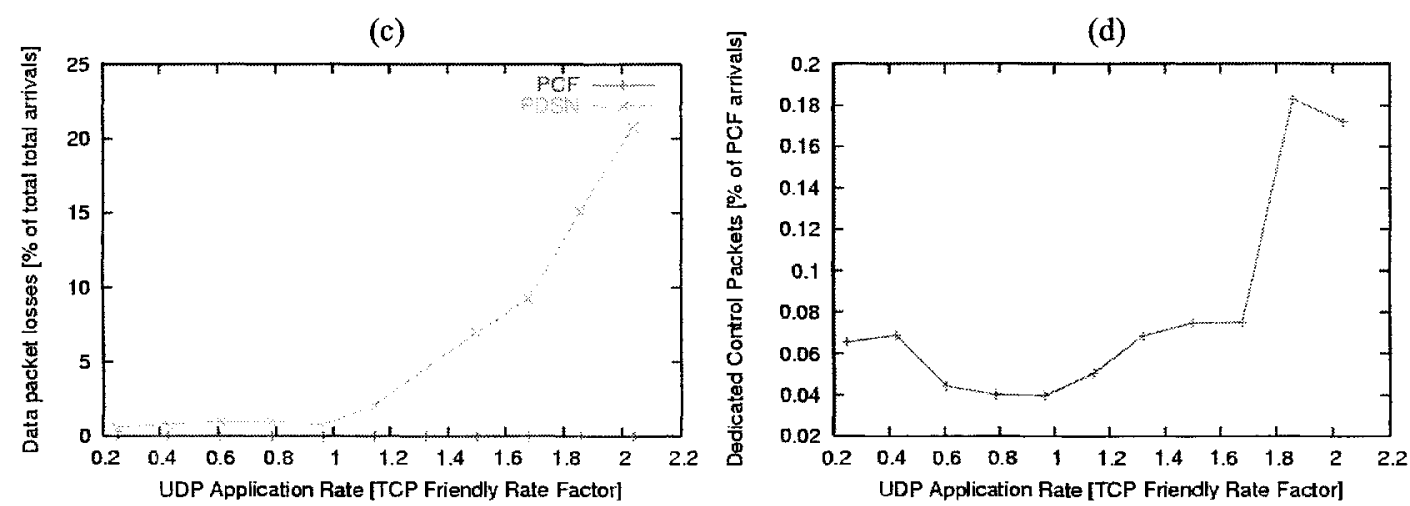

Fig. 7.8 QoS model performance as a function of the UDP application rate (a) average delay during congestion (b) percentage of data packet loss volume at the PDSN per traffic

class (c) Data packet loss volume distribution between the PCF and the PDSN (d) backpressure control packet overhead

There are no data packets drops at the PCF with all UDP load levels at Fig. 7.8c. The PCF queue average fill is controlled to be below the RED, however with increased UDP loads the PCF experience an increase in the average delays (Fig. 7.8a) and an increase of 
control percentage (Fig. 7.8d). Note that the higher UDP loads values ( $>1.8 \times$ TCP friendly) at Fig. 7.8d are larger than the PCF out-link bandwidth, which presents an extreme condition that is recommended to avoid.

\subsection{Conclusions}

In this chapter we enhanced the CDMA-2000 data networks with QoS model for service differentiations. The model adheres to the CDMA-2000 the core node's limitation of low processing power, and the constraint to maintaining the complexity at that node. The model uses the Adaptive-Xoff/Xon mechanism to push congestion towards the edge node, where DiffServ architecture is deployed and provides service differentiations. The DiffServ architecture uses multiple RED physical queues, each assigned to a specific traffic class.

The simulation results demonstrated the service differentiations offered by our QoS model. It shows consistent throughput and packet drop rate differentiations between traffic classes for RED queues at the PDSN that are tuned with equal average delays. The results with multi-transport layers showed that our QoS model improved the fairness between the UDP and the TCP based traffic in terms of bandwidth division as compared with a CDMA-2000 data network with a single level of traffic handling. The backpressure mechanism showed to adequately control the PCF queue fill via limiting the PDSN transmission rate. 


\section{Conclusions and Future Study}

This chapter discusses the findings in previous chapters and provides a summarized conclusion for this study. Ideas for possible future study in this area are also presented.

\subsection{Theoretical Analysis of RED}

Chapter 4 aims to theoretically evaluate the possible RED tune-ups for CDMA-2000 data-networks with the wireless links rate swings. The rate swing repeats each time highrate supplemental-channels are closely allocated at the CDMA-2000 data-network finiteburst mode scheduling. This causes repeated occupancy variations at the bottleneck router's shared-queue, large bursts of overflow-based deterministic drops, and consequential system performances degradation. We propose a mathematical model for the system transient response to explain the performance degrading effects. The model connects the parameters of the CDMA-2000 system, a RED shared-queue, and the traffic build of homogeneous TCP connections with backlogged FTP sources. A non-linear closed-form estimator on the volume of the data packets burst of losses due to buffer overflowing is derived from the model. It allows us to reveal the deployed RED sharedqueue's setting ranges for which performances degrading effects are imminent, and distinguish those from preferred setting ranges.

Numerical solutions to the losses-volume estimate are provided. The results show the setting ranges for the RED controls that are not recommended, which are characterized with excessive packet loss volume of 3 or more packets on the average per connection in a single burst of packet drops. Excessive losses-volumes were shown for low values of the buffer-size, all range of thresholds ratio, low averaging weight, and large values of target queue-size. In particular, setting RED according to the literature recommendations shows excessive overflow loss volume.

Low values for our estimate suggest preferred areas of operation for RED in which the calculated losses-volume estimate is small such that it possibly allows the TCP senders to 
avoid the costly RTO based recovery. Large queue buffer-sizes show small estimate values and indicate the absence of an overflow scenario at the queue, but at the undesirable cost of extensive allowable delay-jitter at the queue. Varying thresholds ratios show only moderate loses estimate changes. Furthermore, some potential expenses accompany such thresholds varying. Low values of the RED minimum thresholds for very low rations result with packet drops at low queue fill for bursty traffic. Large ratios may allow large queue occupancy oscillations at load changes during steady state. Low queue target-size values also achieve losses-volume estimate improvement, however constraining it is not advised as it presents the network-administrative steady-state tradeoff between average-delay and throughput. Large RED averaging weight values present a profound small losses estimate. The absence of overflow-based drops with averaging weight of 1 suggests to implement the queue without the averaging mechanism, allowing RED to stabilize the queue using uniformly distributed random drops and potentially avoid the TCP senders' prolong timeout based recovery. A moderate associated cost is the smaller allowable burst size at the queue from an uncongested queue.

RED's inability to handle properly the transient congestion with random drops during the CDMA-2000 finite-burst mode using the literature tuning recommendations for wired-Internet, and the above listed cost-effect for each of the RED's parameters varied settings, calls for required alternatives. Clearly, a system architect can choose a preferred cost-effect for the RED tune-up from our above results to accommodate certain system needs or certain traffic characteristics. However, alternatively the above results suggest that another congestion-control mechanism is required for proper overload handling. One such alternative can be another, or modified, AQM at the shared-queue specific for cellular systems that can distinguish ACK compression from traffic sources bursts, at the congestion built-up initial stages. For instance, such a mechanism would require to consider the traffic rate change, rather than the (averaged) queue occupancy alone. Another interesting alternative may utilize our results to complement the RED 
mechanism with a supporting mechanism. One such mechanism can utilize the absence of overflow with large buffer-sizes as shown in the thesis, and use available free bufferspace of the feeding node of the PCF (i.e., the PDSN at Fig. 3.1) as reserved buffer space during high congestion at the PCF by using a feedback flow-control mechanism.

\subsection{RED Tune-Up via Simulations}

In chapter 5 we targeted the evaluation via simulations of the RED mechanism as a function of its controls at the context of the CDMA-2000 bandwidth-swings.

We first presented a comparative evaluation of the most commonly deployed versions of TCP. We showed that Vegas TCP outperforms all with no queue overflowing or system under-utilization periods. Reno TCP most under-performs causing large system under-utilization periods. The performances of Thaoe, NewReno, and Reno with SACK option, range in between the former two cases. Therefore, we concluded Reno TCP to be the focus of interest in the thesis simulations. We demonstrated the Reno dynamics using system temporal throughput. It showed prolong period of deep throughput degradation after the SCHs allocations.

We show adverse performance effects due to the interaction between TCP connections and the non-responsive UDP connections in CDMA-2000 data-networks. The TCP senders repeatedly back off after the SCHs allocations due to the ACK compression and overflow effects. The UDP materializes the back-off intervals to achieve un-proportional queue occupancy. Once the UDP aggregated offered-load became sufficiently large compared to the TCP load, it practically inhibited the TCP senders' slow-start phase by aggressively occupying the shared-queue, driving the TCP connections rate close to zero.

The system and the bottleneck shared-queue areas of operations for high performance as a function of the bottleneck RED queue controls were explored using simulations. Three areas of operation were identified with highest throughput as the main performance metric, whereas the RED tuning recommendations for the wired-Internet reside in the complementing area with the lowest throughput. We formulated the optimal configuration 
of RED as a black-box tune-up problem, and used a dual-stages graphical tune-up via simulation. The outperforming area of operation was identified with low queue-averaging factor of 0.8 RTT down to 0 (i.e., high averaging-weights), and with negligible sensitivity to both queue buffer-size values and thresholds ratios. This area shows throughput increase of up to $25 \%$ for a mix of backlogged FTP sources and interactive TCP sources over TCP connections. It also shows half the data packets drop rate compare to the RED recommendation for the wired-Internet.

The results in chapter 5 confirms the analytical model's numerical results at chapter 4 in which large averaging weights (or even the use of the instantaneous queue size) allow RED to stabilize the queue using uniformly distributed random drops, hence avoiding the large performances degradation due to overflow-based closely clustered drops negative effect on TCP sources. High throughput values were also measured at the simulations for very small queue sizes and averaging weights, which are characterized at the analysis (and comparing simulations there) with large burst size of overflow-based drops. The performance degrading effects due to the large burst of drops in these areas rather comes in the form of queue persistent occupancy variations between empty queue to overflowed queue. Hence, these areas are not recommended for RED tune-up as they are susceptible to known tail-drop disadvantages. Such observed disadvantages include delay jitter as large as the queue's buffer-size, large average-delay close to half the buffer-size regardless of the pre-configured target queue size, and unfairness between connections.

\subsection{Backpressure Feedback Flow-Control}

In chapter 6 we targeted the mitigation of the wireless links rate swings at CDMA-2000 data-network on TCP traffic. We evaluated the 3GPP2 proposal for protecting the bottleneck node by deploying backpressure towards the gateway node.

We proposed a feedback flow-control (i.e., backpressure) mechanism to supplement the nodal RED mechanism at the congested bottleneck node, the PCF. The AdaptiveXoff/Xon feedback mechanism protects the bottleneck queue from overflowing during 
excessive transient congestion using free buffer-space at the superior feeding node, the PDSN, whereas a bottleneck's RED mechanism ensures convergence to the desired queue metrics (e.g., average delay) at steady state. The Adaptive-Xoff/Xoff enhances the traditional Xoff/Xon mechanism to introduce new terms: target threshold levels, overflow (or upper threshold overshooting) prediction, adaptive upper threshold, rate throttling, and implicit Xoff elapse time.

We analyzed the backpressure model performances using CDMA-2000 system simulations, and showed the tradeoffs relative to nodal RED and the PCF. We analyzed the hybrid model's temporal dynamics, and showed that in the context the tandem-queues the queues' occupancy variations do not translate to packet delay variations. The hybrid model persistently showed higher throughput, lower packet drop rates, and absence of overflow-based drops, with variable traffic loads and durations of wireless links' 'burst' interval.

We observed the pertaining costs of the backpressure in CDMA-2000. The hybrid model shows an overhead of control packet that is low compared to the traversing data traffic it supports. Larger average-delays were observed at the tandem queues. The throughput increase achieved by the hybrid model is smaller than the increase of the incurred delays, percentage-wise, resulting with power values of the hybrid model lower than these of the nodal model. This suggests that the 3GPP2 proposal for backpressure in CDMA-2000 brings combined performances that are degraded compared to the nodal model. However, if throughput improvement is of specific interest, then the hybrid model suggests a simple architecture to harness already deployed buffers at the PDSN and using available internodes connections (A10) for confronting excessive transient congestions. In the context of throughput improvement, we compare the hybrid model with an alternative nodal model built of a PCF with enlarged buffer size and RED thresholds tuned to match the characteristics of the tandem queues in the hybrid model. The hybrid model showed to surplus the throughput of the enlarged PCF for the larger part of tested burst intervals of the CDMA-2000 finite-burst mode. Furthermore, the hybrid model 
shows a power-curve that matches this of the enlarged PCF for the finite-burst mode burst intervals, and surpluses it for the infinite-burst mode tested burst intervals.

Further research direction is suggested in the area of backpressure in CDMA-2000 routers as follows. The overhead of the backpressure's control packets can be further reduced by piggybacking backpressure feedback indications at GRE encapsulations of the packets flowing in the CDMA-2000 reverse direction. These packets may be at the form of ACKs, or data-packets for bi-directional traffic. The effect of such a delayed encapsulation, with a corresponding algorithm that bounds the waiting time for a packet to traverse the reverse direction, on the Adaptive-Xoff/Xon needs to be evaluated.

\subsection{Backpressure Enabled QoS Model}

In chapter 7 we target the enhancement of CDMA-2000 data networks with QoS model for service differentiation. The proposed QoS solution differentiates services in terms of the relative portion of access to network's shared resources and per traffic class RED queue treatment. It is capable of providing service differentiation in terms of relative throughput, packet loss rate, average delay, and delay jitter, subject to the model's settings. The model employs a DiffServ edge node with multiple physical RED queues, and an internodes backpressure mechanism. The backpressure mechanism is used to push congestion conditions from the bottleneck node (the PCF) towards its superior feeding node (the PDSN) where superior DiffServ based traffic handling can be provided. We provide recommendations for the PDSN RED queues set-up for achieving desired levels of delay and jitter.

We verified our solution with various system and traffic scenarios using simulations. The results show that the backpressure-based QoS model offers differentiated services while adhering to the system limitations. The throughput results show a consistent differentiation between the classes, which can be approximated with the bandwidth division enforced by the packet scheduler at the PDSN. The results also show a sustainable drop rate differentiation achieved by the bandwidth allocation based 
differentiation combined with the multiple RED queues settings. The simulations confirm our RED queues setting recommendation for achieving desired levels of average delay at the PDSN queues.

The results with multi-transport layers show that our QoS model improved the bandwidth division between the UDP and the TCP based traffic compared to a CDMA2000 data system with a single level of traffic handling. The model allows the TCP to properly obtain its fair bandwidth shared during slow-start and loss recovery phases. However the lower requested bandwidth from TCP flows due to existing bandwidth swings and their effect of the TCP congestion-control mechanism allows the WRR scheduler at the DiffServ architecture to correctly re-allocate un-used bandwidth to the persistent UDP traffic, resulting with higher throughput for UDP based flows.

The backpressure mechanism showed to adequately control the PCF queue fill via limiting the PDSN transmission rate. The backpressure thresholds were tuned below the PCF RED thresholds for the purpose of pushing congestion to PDSN, in contrast to above the RED thresholds at chapter 6 for the purpose of overflow events protection. The results showed that no data packets are dropped at the PCF with large range of traffic loads. The PCF queue is stabilized to low occupancy levels while incurring low variations. The backpressure control overhead is shown to be low compared to the data traffic volume that it supports. The achieved low PCF average delays were shown to be significantly lower than the PDSN queues delays such that it can be incorporated in the total class-based delay to achieve a desired differentiated class-based target delay during congestion.

The QoS model showed little effect on the CDMA-2000 finite-burst mode base bandwidth swings phenomenon. It is rather demonstrated that it can provide differentiated services despite the bandwidth swings. Moreover, the sum performance of all traffic aggregates (e.g., throughput) is shown to be comparable with the results obtained at chapter 6 for a CDMA-2000 system of a single level of service. 
Further research directions for enhancing our backpressure QoS model are as follows. First, the proposed solution targets single sided traffic flowing at the CDMA-2000 forward direction, as common with $3 \mathrm{G}$ cellular data systems. Consecutively, our model provides service differentiation in one direction of traffic flow, and is therefore asymmetric. Development of a complementary symmetric architecture to support next generation $4 \mathrm{G}$ systems' dual-sided traffic is a topic for a future research. Second, our model can be upgraded to a full DiffServ domain with the PCF node as a DiffServ capable core node once the PCF infrastructure can support it. In this model multiple physical queues are to be deployed at the PCF core node as well, and a backpressure instance between each of the PCF queues to the corresponding class queue at the PDSN. The backpressure instances in such a scheme could be tuned to rather high queue occupancy at the PCF queues for focusing on protecting the PCF queues at excessive congestion scenarios using PDSN spare buffers. 


\section{Bibliography}

[1] K. Beomjoon and L. Jaiyong, "Analytic Models of Loss Recovery of TCP Reno with Packet Losses”, H.-K. Kahng (Ed.): ICOIN 2003, LNCS 2662, pp. 938-948, Aug. 2003

[2] TIA/EIA/IS-2000.1, "Introduction to cdma2000 standards for spread spectrum systems", Mar. 1999

[3] TIA/EIA/IS-2000.5-A, "Upper Layer (Layer 3) Signaling Standard for cdma2000 Spread Spectrum Systems", Mar. 2000

[4] V. Paliwal, P. Larijani, I. Lambadaris, and B. Nandy, "Effect of Channel Variation in IP/cdma2000 Interconnection Performance", of the IEEE International Conference on Communications (ICC 05), Seoul, Korea, May 16-20, 2005

[5] M. Chan and R. Ramjee, “TCP/IP Performance over 3G Wireless Links with Rate and Delay Variation" in Proc. of ACM Mobicomm, Feb. 2002

[6] S. Floyd and V. Jacobson, "Random Early Detection Gateways for Congestion Avoidance", IEEE/ACM Transactions on Networking, v.1 n.4: 397-413, Aug. 1993

[7] S. Floyd, "Optimum functions for computing the drop probability", http:/www.aciri.org/floyd/REDfunc.txt, November 1997, last reviewed on Sep.28 2006

[8] S. Floyd, "RED: Discussions of Setting Parameters", http://www.aciri.org/floyd/REDparameters.txt, November 1997, last reviewed on Sep.28 2006

[9] S. Floyd and K. Fall, "Promoting the use of end-to-end congestion control in the Internet", IEEE/ACM Transactions on Networking, v.7 n.4, pp.458-472, Aug. 1999 
[10] S. Floyd, R. Gummadi, and S. Shenker, "Adaptive RED: An Algorithm for Increasing the Robustness of RED's Active Queue Management”, AT\&T Center for Internet Research at ICSI, Aug. 2001

[11] M. May, J. Bolot, C. Diot, and B. Lyles, "Reasons Not to Deploy RED", in Proc. of 7th. International Workshop on Quality of Service, pp. 260-262, Jun. 1999

[12] T. Ziegler, S. Fdida, and C. Brandauer. "Stability Criteria for RED with Bulk-data TCP Traffic”, IFIP ATM \& IP Working Conference, Budapest, Jun. 2001

[13] Y. Liu, F. L. Presti, V. Misra, D. Towsley, and Y. Gu, "Scalable Fluid Models and Simulations for Large-Scale IP Networks", ACM Transactions on Modeling and Computer Simulation, v. 14, n. 3, pp. 305-324, Jul. 2004

[14] V. Misra, W. Gong, and D. Towsley, "A fluid-based analysis of a network of AQM routers supporting TCP flows with an application to RED", in Proc. of ACM SIGCOMM'00, Stockholm, Sweden, Sep. 2000

[15] M. Christiansen, K. Jeffay, D. Ott, and F. D. Smith, "Tuning RED for Web Traffic", SIGCOMM, pp. 139-150, Sep. 2000

[16] S. Hanks, T. Li, D. Farinacci, and P. Traina, "RFC1701 - Generic Routing Encapsulation (GRE)", Oct. 1994

[17] S. Sayeedi and A. Farcasanu, “A10/A11 Flow Control", A30-200420040920, IOS v5.0 Contribution, 3GPP2, Motorola, Sep. 2004

[18] M Vasudevan and S. Nesargi, "Nortel comments on the Motorola flow-control proposal", A20-20030818-014, TSG-A Contribution, 3GPP2, A20-20030818-014, Nortel Networks, Feb. 2005

[19] M. Gerla and L. Kleinrock, "Congestion Control in Interconnected LANs", IEEE Network, Vol. 2 Iss.1, pp. 72-75, 1998 
[20] K. K. Ramakrishnan and R. Jain, "A binary feedback scheme for congestion avoidance in computer networks", ACM Transactions on Computer Systems (TOCS), v. 8, n. 2, pp. 158-181, May 1990

[21] J. Wechta, A. Eberlein, and F. Halsall, "The Interaction of TCP Flow Control Procedure in End Nodes on the Proposed Flow Control Mechanism for Use in IEEE 802.3 Switches", HPN 98, Vienna, Austria, 1998

[22] J. Wechta, A. Eberlein, F. Halsall, and M. Spratt, "Simulation Based Analysis of the Interaction of End-to-End and Hop-by-Hop Flow Control Schemes in Packet Switched LANs", in Proc. of the 15th UK Teletraffic Symposium on Performance Engineering in Information Systems, Durham, UK, Mar. 1998

[23] Y. T. Wang and B. Sengupta, "Performance Analysis of a Feedback Congestion Control Policy Under Non-Negligible Propagation Delay", ACM, 1991

[24] J. Wechta, A. Eberlein, F. Halsall, “An Investigation into the Performance of Switched LANs", in Proc. of the Conference on Networks and Optical Communications, Manchester, UK, 1998

[25] C. Ozveren, R. Simcoe, and G. Varghese, "Reliable and Efficient Hop-by-Hop Flow Control", in Proc. of ACM Sigcomm, v. 24 n. 4, pp. 89-100, 1998

[26] C. M. Pazos, C. Juan, S. Agrelo, and M. Gerla, "Using Backpressure to Improve TCP Performance with Many Flows", UCLA, in Proc. of IEEE INFOCOM NY, 1999

[27] Y. Bernet, D. Durham, and F. Reichmeyer, "Requirements of DiffServ Boundary Routers", IETF Internet Draft, draft-bernet-diffedge-01.txt, Nov. 1998

[28] K. Nichols, S. Blake, F. Baker and D. Black, "Definition of the Differentiated Services Field (DS Field) in the IPv4 and IPv6 Headers", RFC 2474, Dec. 1998

[29] S. Blake, D. Black, M. Carlson, E. Davies, Z. Wang, and W. Weiss. “An Architecture for Differentiated Services” RFC 2475. Dec. 1998 
[30] Y. Bernet, J. Binder, S. Blake, M. Carlson, S. Keshav, E. Davies, B. Ohlman, D. Verma, Z. Wang, and W. Weiss, "A Framework for Differentiated Services", IETF Internet Draft, draft-ietf-diffserv-framework-01.txt, Oct., 1998

[31] J. Ibanez and K. Nichols, "Preliminary Simulation Evaluation of an Assured Service", IETF Internet Draft, draft-ibanez-diffserv-assured-eval-00.txt, Aug. 1998

[32] K. Hyogon, E. Leland, and E. Thomson, "Evaluation of Bandwidth Assurance Service using RED for Internet Service Differentiation", INFOCOM, Mar. 1999

[33] The Network Simulator NS-2, http://www.isi.edu/nsnam/ns/, University of Southern California, Information Science Institute, last reviewed on Sep. 29, 06

[34] S. Floyd and V. Jacobson, "On Traffic Phase Effects in Packet-Switched Gateways", Internetworking: Research and Experience, v.3 n.3, pp.115-156, Sep. 1992 